\title{
Facilitating sensor interoperability and incorporating quality in fingerprint matching systems
}

\author{
Rohan D. Nadgir \\ West Virginia University
}

Follow this and additional works at: https://researchrepository.wvu.edu/etd

\section{Recommended Citation}

Nadgir, Rohan D., "Facilitating sensor interoperability and incorporating quality in fingerprint matching systems" (2006). Graduate Theses, Dissertations, and Problem Reports. 1738.

https://researchrepository.wvu.edu/etd/1738

This Thesis is protected by copyright and/or related rights. It has been brought to you by the The Research Repository @ WVU with permission from the rights-holder(s). You are free to use this Thesis in any way that is permitted by the copyright and related rights legislation that applies to your use. For other uses you must obtain permission from the rights-holder(s) directly, unless additional rights are indicated by a Creative Commons license in the record and/ or on the work itself. This Thesis has been accepted for inclusion in WVU Graduate Theses, Dissertations, and Problem Reports collection by an authorized administrator of The Research Repository @ WVU. For more information, please contact researchrepository@mail.wvu.edu. 


\title{
Facilitating Sensor Interoperability and Incorporating Quality in Fingerprint Matching Systems
}

\author{
by \\ Rohan D. Nadgir \\ Thesis submitted to the \\ College of Engineering and Mineral Resources \\ at West Virginia University \\ in partial fulfillment of the requirements \\ for the degree of \\ Master of Science \\ in \\ Electrical Engineering \\ Arun Ross, Ph.D., Chair \\ Lawrence Hornak, Ph.D. \\ Xin Li, Ph.D. \\ Lane Department of Computer Science and Electrical Engineering \\ Morgantown, West Virginia \\ 2006
}

Keywords: Biometrics, Fingerprint sensors, Fingerprint Sensor Interoperability, Inter-sensor distortion, Thin Plate Splines, Feature Quality, Score formulation, Image quality, Gabor filters 


\begin{abstract}
Facilitating Sensor Interoperability and Incorporating Quality in Fingerprint Matching Systems by

Rohan D. Nadgir

Master of Science in Electrical Engineering

West Virginia University

Arun Ross, Ph.D., Chair

This thesis addresses the issues of sensor interoperability and quality in the context of fingerprints and makes a three-fold contribution. The first contribution is a method to facilitate fingerprint sensor interoperability that involves the comparison of fingerprint images originating from multiple sensors. The proposed technique models the relationship between images acquired by two different sensors using a Thin Plate Spline (TPS) function. Such a calibration model is observed to enhance the inter-sensor matching performance on the MSU dataset containing images from optical and capacitive sensors. Experiments indicate that the proposed calibration scheme improves the inter-sensor Genuine Accept Rate (GAR) by $35 \%$ to $40 \%$ at a False Accept Rate (FAR) of $0.01 \%$. The second contribution is a technique to incorporate the local image quality information in the fingerprint matching process. Experiments on the FVC 2002 and 2004 databases suggest the potential of this scheme to improve the matching performance of a generic fingerprint recognition system. The final contribution of this thesis is a method for classifying fingerprint images into 3 categories: good, dry and smudged. Such a categorization would assist in invoking different image processing or matching schemes based on the nature of the input fingerprint image. A classification rate of $97.45 \%$ is obtained on a subset of the FVC 2004 DB1 database.
\end{abstract}


I dedicate my thesis to my family and friends 


\section{Acknowledgments}

I appreciate the opportunity provided to me by Dr. Ross to work with him as a graduate student. It has been an honor to have him as my research advisor. His work ethics and attitude have been a total inspiration. I have learned a lot from his determination, dedication and ability to prioritize things. I would have not reached this phase of my life without his guidance and constant motivation.

I would also like to thank Dr. Li and Dr. Hornak, my committee members, for their suggestions and guidance. I would be forever grateful to Dr. Hornak and Dr. Ross for giving me the opportunity to join West Virginia University and transfer from Drexel University.

I appreciate the financial support provided by Center for Identification Technology Research (CITeR) for certain parts of my thesis. I would like to thank Dr. Anil Jain of Michigan State University for granting us access to the MSU Fingerprint Database. I am grateful to Dr. Stephanie Schuckers and Dr. Sunil Kumar of Clarkson University for their discussions.

I would like to take this opportunity to thank my friends and lab-mates for the great times I have experienced at the Biometric Systems Lab. I would like to express my gratitude to Sarvesh Makthal for helping me in getting started with my research, Christopher Boyce for the constructive criticism and pointers regarding my research as well as Kiran Tadaka for lending a hand with the SDK's of the commercial fingerprint systems. I will always remember Shigefumi Yamada, Samir Shah, Simona Crihalmeanu, Kiran Tadaka, Jidnya Shah, Phani Ivatury, Rajiv Mukherjee and Nikhil Burri for their co-operation, their feedback on my research and also for the fun we had together.

I will be forever indebted to my parents and brother for their love and never-ending support. I will always remember the sacrifices they have made on my behalf. 


\section{Contents}

Acknowledgments iv

List of Figures vii

List of Tables 18

1 Introduction $\quad 1$

1.1 Introduction . . . . . . . . . . . . . . . . . . . . . 1

1.2 Problem Statement . . . . . . . . . . . . . . . . . . . . . . 2

1.2 .1 Sensor Interoperability . . . . . . . . . . . . . . . . . . . . . . . . . . . . 5

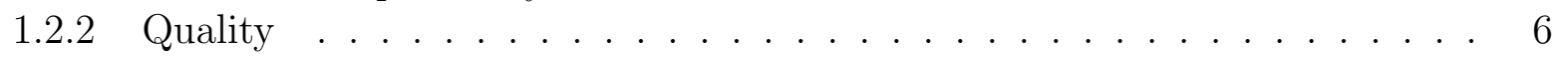

1.3 Motivation . . . . . . . . . . . . . . . . . . . . 6

$1.3 .1 \quad$ Sensor Interoperability $\ldots \ldots \ldots \ldots \ldots \ldots$

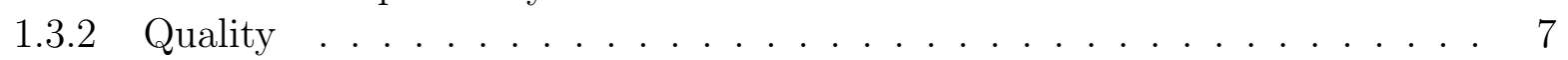

1.4 Contributions of this thesis $\ldots \ldots \ldots \ldots \ldots$

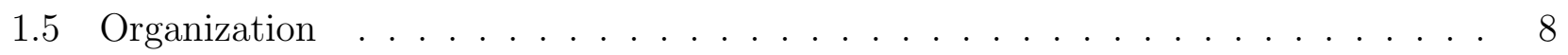

2 Sensor Interoperability 10

2.1 Introduction . . . . . . . . . . . . . . . . . . . . . . . 10

2.2 Fingerprint Sensing Technology . . . . . . . . . . . . . . . . . . . 13

2.2 .1 Optical . . . . . . . . . . . . . . . . . . . . 13

2.2 .2 Capacitive . . . . . . . . . . . . . . . . . . . . . . 15

2.2 .3 Piezoelectric . . . . . . . . . . . . . . . . . . . . . . . . 15

2.2 .4 Temperature Differential . . . . . . . . . . . . . . . . . . 15

2.2 .5 Ultrasound . . . . . . . . . . . . . . . . . . . . . . . . . 16

2.2 .6 Touchless $\ldots \ldots \ldots \ldots \ldots \ldots$

2.2 .7 Multispectral . . . . . . . . . . . . . . . . . . . . . 16

2.2 .8 Distortions introduced in different sensing technologies . . . . . . . . . . . 17

2.3 Sensor Interoperability . . . . . . . . . . . . . . . . . . . . . 17

2.4 Thin Plate Spline model . . . . . . . . . . . . . . . . . . . . . . . . 21

2.4 .1 Bending Energy of TPS . . . . . . . . . . . . . . . . . . . . 21

2.4 .2 Determining the average pixel deformation . . . . . . . . . . . . . . 23

2.4 .3 Orientation computation . . . . . . . . . . . . . . . . . . . 23

2.4 .4 Derivation of scaling parameters . . . . . . . . . . . . . . . . . . . 24

2.5 Experimental Results . . . . . . . . . . . . . . . . . . . . . . . . . . 24

2.5.1 Role of scaling parameters . . . . . . . . . . . . . . . . . . . 29

2.6 Summary and Future Work _. . . . . . . . . . . . . . . . . . . . . . 34 
3 Fingerprint Image Quality $\quad 35$

3.1 Introduction . . . . . . . . . . . . . . . . . . . . . . . 35

3.2 Quality Estimation Techniques . . . . . . . . . . . . . . . . 36

3.2 .1 Intensity-based Quality Estimation . . . . . . . . . . . . . . . . 36

$3.2 .2 \quad$ Filter-based Quality Estimation . . . . . . . . . . . . . . . . . . . . . . . . . . . . . . . . . . . .

3.2 .3 Classifier-based scheme . . . . . . . . . . . . . . . . . . . . . . . . 39

3.2.4 Wavelet-based Quality Estimation . . . . . . . . . . . . . . . . . . . 41

$3.2 .5 \quad$ Hybrid Approaches . . . . . . . . . . . . . . . . . . . . . . . . . 42

3.3 Gabor Analysis . . . . . . . . . . . . . . . . . . . . . . . . . . . . 45

3.3 .1 Structure of $2 \mathrm{D}$ Gabor Filter . . . . . . . . . . . . . . . . . . . . . . . . . . . . . . . . . . . . . . .

3.3 .2 Advantages of Gabor Filter . . . . . . . . . . . . . . . . . . . 46

3.3 .3 Variations from the standard Gabor approach . . . . . . . . . . . . . . . . 46

3.3.4 Gabor-based Quality Classification . . . . . . . . . . . . . . . . . . 47

3.3 .5 Summary and future work . . . . . . . . . . . . . . . . . 58

3.4 Incorporating estimated quality into a fingerprint recognition system $\ldots . . .58$

3.4 .1 Incorporating quality at different stages of the recognition system . . . . . 58

3.4.2 Proposed incorporation of extracted Feature Quality into Matching Score

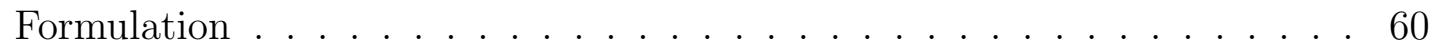

3.4 .3 Conclusion . . . . . . . . . . . . . . . . . . 66

4 Summary and Future Work $\quad 67$

4.1 Sensor Interoperability $\ldots \ldots \ldots \ldots$. . . . . . . . . . . . . 67

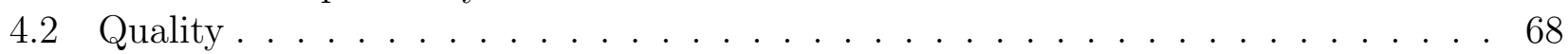

\begin{tabular}{|lr}
\hline Appendices & 69
\end{tabular}

\begin{tabular}{|ll} 
A Roll versus Plain Prints: An Experimental Study & 69
\end{tabular}

A.1 Introduction . . . . . . . . . . . . . . . . . . . . . . . . . . . 69

A.2 Background . . . . . . . . . . . . . . . . . . . . . 69

A.3 Experimental Results . . . . . . . . . . . . . . . . . . . . . . . 74

A.4 Summary . . . . . . . . . . . . . . . . . . . . . . 77

\begin{tabular}{ll}
\hline B Fingerprint Matchers - NIST and VeriFinger & 79
\end{tabular}

$\begin{array}{ll}\text { C Ridge Feature-based matcher: Fingercode } & 80\end{array}$

$\begin{array}{ll}\text { References } & 82\end{array}$ 


\section{List of Figures}

1.1 Fingerprint images with differing qualities and their corresponding extracted minutiae (a) Good quality image, (b) Dry image, (c) Smudged image, (d) Partial print, (e) Rotated image, (f) Image with scar/bruise. . . . . . . . . . . . . . . . . . .

1.2 Visual differences between impressions of the same finger acquired using five different sensors. Verifier 300, Hamster III and U.are.U 4000 are optical sensors. Hamster III is based on SEIR (Surface Enhanced Irregular Reflection) technology, while U.are.U 4000 and Verifier 300 use a FTIR (Frustrated Total Internal Reflection) technology. The USB 2500 is an electro-optical sensor and the 100AX is a capacitive sensor. . . . . . . . . . . . . . . . . . .

2.1 Four different methods for fingerprint acquisition. (a) Rolled print (from the NIST Special Database 4), (b) Dab print (from the FVC 2002 DB1 Database), (c) Swiped sensor print (from the FVC 2004 DB3 Database), (d) Latent print [1] .

2.2 Fingerprint images obtained by different acquisition methodologies. (a) Rolled print (from the NIST Special Database 4), (b) Flat print (from the FVC 2002 DB1 Database), and (c) Slap print $[2] \ldots \ldots \ldots \ldots$. . . . . . . . . . . . . . 11

2.3 Working principles of different sensing technologies . . . . . . . . . . . . . . . 13

2.4 Two different ways of facilitating interoperability. (a) Distortion compensation model and (b) Inter-sensor distortion model. The numbers within circles denote the sequence of steps. . . . . . . . . . . . . . . . . . . 19

2.5 Minutiae correspondence (manually selected) across representative image pairs, serve as inputs to the TPS model. . . . . . . . . . . . . . . . . . . 19

2.6 Manually selected control points from representative image pairs are provided as inputs to the TPS model. The affine and non-linear parameters derived from the average deformation model are used for image and minutiae calibration during the authentication stage. . . . . . . . . . . . . . . . .

2.7 Minutiae calibration: The inter-sensor distortion compensation block is introduced in-between the minutiae extraction and matching modules to handle inter-sensor

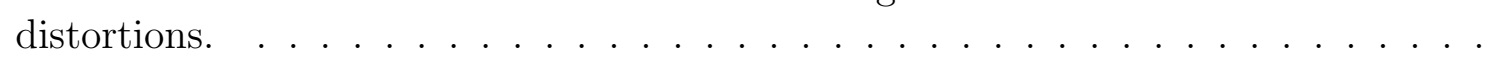

2.8 Minutiae orientation is modified on basis of the transformed minutiae location (after average deformation). $\mathrm{P}$ is the original minutiae point location while $\mathrm{Q}$ is the corresponding 'angle point'. P' and Q' are the corresponding locations of $\mathrm{P}$

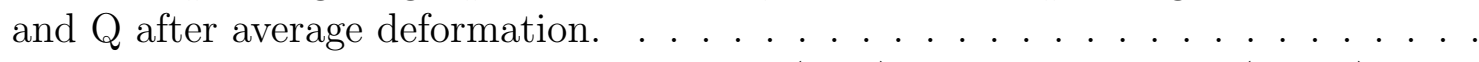

2.9 Sample fingerprints from Digital Biometrics (DBI) and VERIDICOM (VERI) sensors. (a) Image acquired from DBI. (b) Image acquired from VERI. . . . . . . . . 25 
2.10 Fingerprints in the first 2 columns are matched and the corresponding minutiae matching results are shown in the third column. Highlighted minutiae represent the minutiae that have been matched. The first row illustrates DBI vs DBI matching, the second VERI vs VERI matching and the third VERI vs DBI matching.

2.11 The histogram of minutiae points extracted from the DBI and VERI images. . . .

2.12 Demonstration of the calibration process using mesh-grid plots. (a) and (b) The deformation of two VERI images with respect to their corresponding DBI images as estimated by the TPS model. (c) A pre-calibrated VERI image. (d) The postcalibrated VERI image based on the deformation of eight representative image pairs. The dashed rectangle in $(\mathrm{d})$ indicates the dimensions of the original VERI image. . . . . . . . . . . . . . . . . . . . . .

2.13 (a) Original mesh-grid plot with minutiae (b) Mesh-grid plot with calibrated minutiae (after minutiae calibration) (c) Mesh-grid plot with minutiae (after image

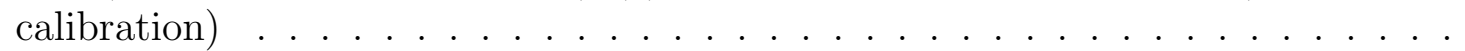

2.14 (a) Original fingerprint image. (b) Image generated after applying non-linear transformation leads to loss of information. . . . . . . . . . . . . . .

2.15 ROC curves indicating the improvement in inter-sensor performance on the MSU database. (a) VeriFinger matcher. (b) BOZORTH3 matcher. . . . . . . . . . . . . 31

2.16 Improved inter-sensor minutiae matching after calibration. (a) indicates the matching minutiae before calibration, (b) and (c) show the matching minutiae after image and minutiae calibration, respectively. Matching minutiae pairs are highlighted for distinguishing them from the non-matching minutiae pairs. Match scores as assessed by the VeriFinger matcher for (a), (b), (c) are 35, 193 and 202, respectively. . . . . . . . . . . . . . . . . . .

2.17 Plot indicating the distribution of weights for the non-linear parameters (derived using TPS) for each of the 8 representative images. (a) Weights computed in the horizontal direction. (b) Weights computed in the vertical direction. The $\mathrm{x}$-axis corresponds to the representative image pair while the y-axis represents the weights of the non-linear parameters.

2.18 ROC curves comparing the minutiae scaling performance with non-linear image and minutiae calibration. (a) VeriFinger matcher. (b) BOZORTH3 matcher. . . .

3.1 (a) Original Fingerprint, (b) Segmented Fingerprint .

3.2 Gabor filters with the same orientation but different inter-ridge distances (frequencies) (a) 6 pixels, (b) 7 pixels, (c) 8 pixels, (d) 9 pixels, (e) 10 pixels. . . . . . 48

$3.3 \quad$ Flowchart for gabor-based quality classification approach . . . . . . . . . . . . . . 49

3.4 Fingerprint foreground segmentation of (a) Good image (c) Dry image (e) Smudged image and their corresponding segmented images (b), (d), (f), respectively. . . .

3.5 Intermediate results for the gabor-based classification approach (a) Original image (b) Segmented image (c) Maximum gabor response image (d) Histogram of gabor pixel responses.

3.6 Pixel-based gabor response of good quality images shown in (a) $\operatorname{good}_{1}$ and (b) $\operatorname{good}_{2}$. (c) and (d) show the corresponding maximum gabor response images. Histogram of gabor pixel responses are shown in (e) and (f). . . . . . . . . . .

3.7 Pixel-based gabor response of $d r y$ quality images shown in (a) $d r y_{1}$ and (b) $d r y_{2}$. (c) and (d) show the corresponding maximum gabor response images. Histogram of gabor pixel responses are shown in $(\mathrm{e})$ and $(\mathrm{f}) . \ldots \ldots \ldots$. . . . . . . . . 54 
3.8 Pixel-based gabor response of smudged quality images shown in (a) smudged

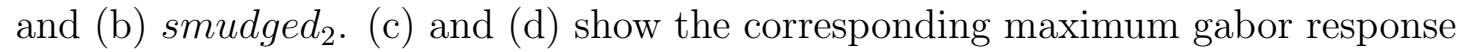
images. Histogram of gabor pixel responses are shown in (e) and (f). . . . . . . . 55

3.9 Classification of good, dry and smudged quality images using the $\left\{X_{\lambda_{1}}, X_{\lambda_{1} \lambda_{2}}, X_{\lambda_{2}}\right\}$ feature set. . . . . . . . . . . . . . . . . . . . 57

3.10 Flowchart of a minutiae-based matching algorithm. Modified flowchart for incorporating feature quality into the score formulation is indicated by the dotted lines. . . . . . . . . . . . . . . . . . . . .

3.11 Minutiae-based matcher: Improvement in performance can be observed by comparing the proposed feature quality-based score formulation with the standard score formulation for multiple databases from FVC 2004 and 2002 . . . . . . . . .

3.12 Flowchart of the fingercode based matching algorithm - (a) Original (b) Modified flowchart after incorporating feature quality into the score formulation . . . . . .

3.13 Ridge feature-based matcher: Improvement in performance can be observed by comparing the proposed feature quality-based score formulation with the standard score formulation for multiple databases from FVC 2004 and 2002 . . . . . . . . .

A.1 Fingerprint images obtained by different acquisition methodologies. (a) Rolled print (from the NIST Special Database 4); (b) Dab print (from the FVC 2002

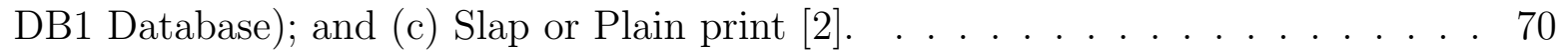

A.2 Illustration of a fingerprint card available in the NIST Special Database 29 [3]. . . 72

A.3 Segmented images for right thumb, left thumb, and the four left plain impressions [3]. 72

A.4 Plain impressions are segmented into individual fingers $[4] . \ldots . \ldots . . . . .73$

A.5 Histograms illustrating the number of minutiae points for the roll and plain (slap) prints corresponding to the ten fingers. (a) Right Thumb, (b) Left Thumb, (c) Right Index finger, (d) Left Index finger, (e) Right Middle finger, (f) Left Middle finger, (g) Right Ring finger, (h) Left Ring finger, (i) Right Little finger, (j) Left Little finger. . . . . . . . . . . . . . . . . . . . . 76

A.6 The verification performance summarized using ROC curves for the SD29 database. The NIST matcher was used to generate the genuine and impostor scores. Note that the roll-versus-roll matching performance is significantly superior possibly due to the large number of minutiae points available for matching. . . . . . . . . . 77

A.7 CMC curves on the SD29 database as assessed using the NIST matcher. In this experiment, the roll-versus-plain identification accuracy is comparable to the other two scenarios, viz., roll-versus-roll and plain-versus-plain. . . . . . . . . . . . . . .

C.1 Ridge feature-based matcher: Flowchart of fingerprint matching system (taken from $[5]) \ldots \ldots \ldots \ldots 1$ 


\section{List of Tables}

$2.1 \quad$ Fingerprint Sensing Technologies. . . . . . . . . . . . . . . . . . . . . . . . . 14

2.2 TPS scaling parameters for the representative images $\ldots \ldots \ldots$

3.1 Percentage of pixels defined by the thresholds $\lambda_{1}$ and $\lambda_{2}$, used for image quality

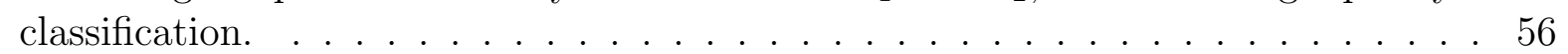

3.2 Confusion Matrix based on Naive-Bayes classifier (ten-fold validation) $\ldots . . .57$ 


\section{Chapter 1}

\section{Introduction}

\subsection{Introduction}

Biometrics refers to the use of distinctive physical (e.g., fingerprints, face, retina, iris, hand geometry, palm) and behavioral (e.g., gait, signature, speech) characteristics for automatically recognizing individuals [6]. Fingerprints have been extensively used in forensic applications for identifying criminals and also in commercial applications for automatic authentication. Fingerprints are considered to be reliable due to their uniqueness and permanence [7]. Fingerprint recognition is being used in a variety of applications including the US-VISIT ${ }^{1}$ program instituted by the Department of Homeland Security (DHS) and the IAFIS ${ }^{2}$ developed by the FBI.

Fingerprints are oriented texture patterns consisting of ridges and valleys present on the surface of an individual's finger. These texture patterns are created by ridges and valleys that alternate and flow in a locally constant direction [8]. A fingerprint can be represented by features that lend themselves to matching. These extracted features are matched using various different methodologies and most of them can be coarsely classified into three families [6].

1. Correlation-based matching: Correlation between corresponding pixels of two different superimposed fingerprint images is computed. Here, the fingerprint image pixels constitute the feature set and contribute toward matching.

2. Minutiae-based matching: Each fingerprint is represented by a collection of extracted features, namely minutiae points. Minutiae points are anomalies detected or observed in the

\footnotetext{
${ }^{1}$ United States Visitor and Immigration Status Indicator Technology

${ }^{2}$ Integrated Automated Fingerprint Identification System
} 
ridges - ridge endings and ridge bifurcations.

3. Ridge feature-based matching: Features extracted from the fingerprint ridge pattern (e.g., local orientation and frequency, ridge shape, texture information etc.) are used for matching. Both, the correlation-based matcher and minutiae-based matcher can be considered to be sub-families of ridge feature-based matching as the minutiae and pixel intensities are actually a representation of the fingerprint ridge pattern.

\section{$1.2 \quad$ Problem Statement}

Fingerprint matching is the process in which the features extracted from two fingerprints are compared by an algorithm and a degree of similarity or match score is returned. If multiple instances of the same fingerprint are matched, also termed as genuine matching or intra-class matching, then a high similarity score is expected as compared to the one observed across different fingerprints, also referred to as imposter matching or inter-class matching. Many times, intraclass matching results in a poor match score which can occur due to various reasons as listed below [6]:

- Rotation and Translation - The same finger may be placed at different locations on the platen across different captures, leading to a global translation effect across different captures. Also, across captures the same finger can be placed on the sensor at different angles, introducing a rotation effect.

- Partial overlap - Due to translations and rotations across captures, different impressions of the same finger could have only a small overlap between them. This is usually the case with sensors having small capture areas. Due to the small overlap between different impressions of the same finger, the genuine similarity score decreases while the probability of an imposter matching a certain set of features increases.

- Non-linear distortion - Non-linear deformations are primarily introduced during fingerprint acquisition, as the three-dimensional (3D) elastic surface of the finger is pushed against a flat sensor surface. This 3D to 2D mapping leads to non-linear deformations [9]. Varying amounts of non-linear distortion could contribute towards the inability of fingerprint matchers to match different impressions of the same finger successfully. 
- Pressure differences - Applying extra pressure might lead to smudged areas where as applying inadequate pressure might lead to dry areas within a fingerprint. Also, varying pressure differences across the fingerprint can contribute towards pronounced non-linear distortions.

- Changing skin conditions - Depending on the fingerprint skin condition or the amount of sweat present at the time of capture, a smudged or a dry effect within an image can be observed. More sweat leads to smudged fingerprint images while less sweat leads to dry fingerprint images.

- Feature extraction errors - The feature extraction algorithms are imperfect and often introduce measurement errors. Errors could occur at different stages of the feature extraction process, like segmentation of fingerprints, estimation of orientation etc. In a minutiae-based algorithm, spurious minutiae could be detected or the orientations and locations of the detected minutiae could be perturbed in the gray-scale images. Temporary or permanent changes due to scars or bruises could lead to improper feature detection as well.

- Sensor conditions - Dirt or latent prints present on the platen of the sensor can lead to poor quality prints. Also, the process of converting the scanned print into a digital image might introduce noise, e.g., blocky artifacts are observed in capacitive scanners due to inter-plate distances. The ergonomics of each sensor, like ease of use, alignment and positioning etc., can also affect the quality of the captured print [10].

- Sensor Interoperability - Variations are introduced in the images captured across different sensors due to variations in sensing technology, resolutions, sensing areas etc. The inability of the matcher to compensate for such inherent variations leads to a poor matching performance.

In Fig. 1.1 (a-e), the variations introduced in the same fingerprint image due to changes in pressure, dryness, position and rotation in comparison with a good quality fingerprint (Fig. 1.1 (a)) are shown. Alongside are the minutiae detected for each of those prints, and one can visually distinguish the variations introduced across these detected minutiae patterns. It would be very difficult for a fingerprint matcher to compensate for these variations. Fig. 1.1 (f) also shows an image having cuts or bruises.

In this thesis, two of the most important problems observed in fingerprint matching are addressed - sensor interoperability and quality. 

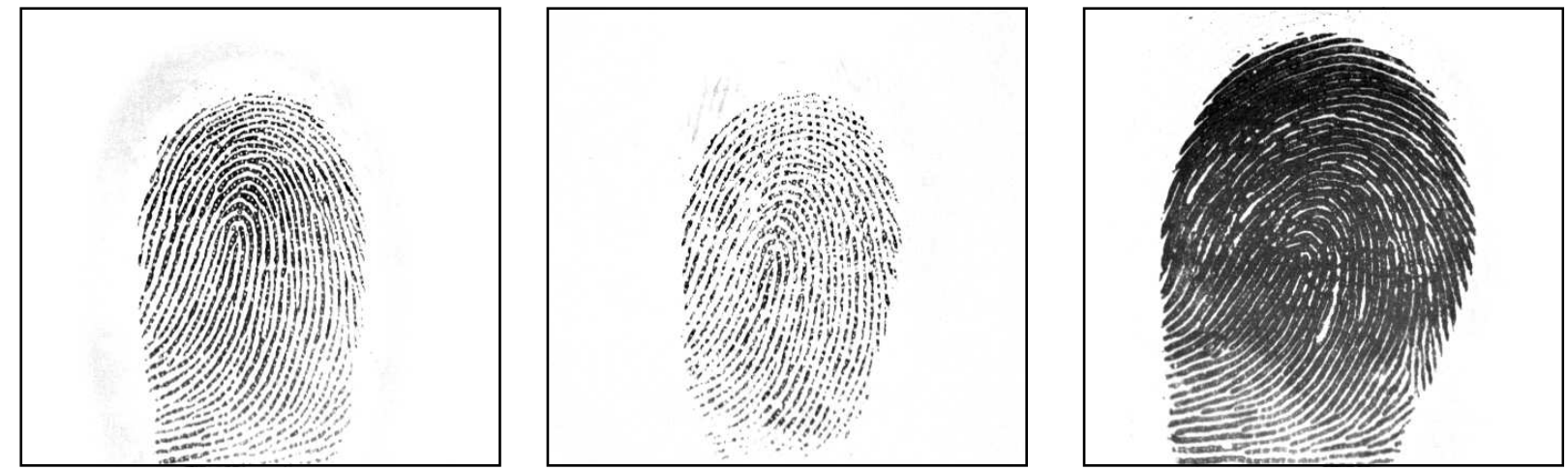

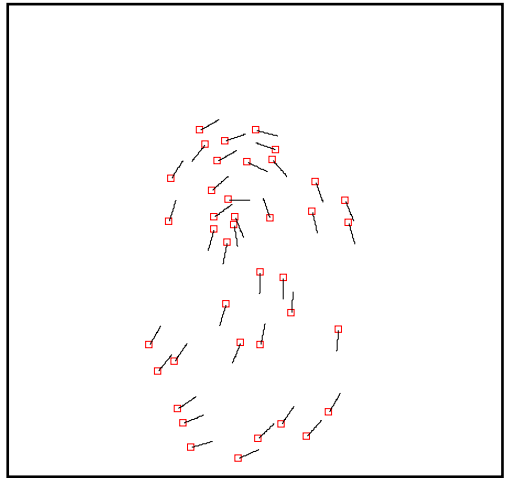

(a)
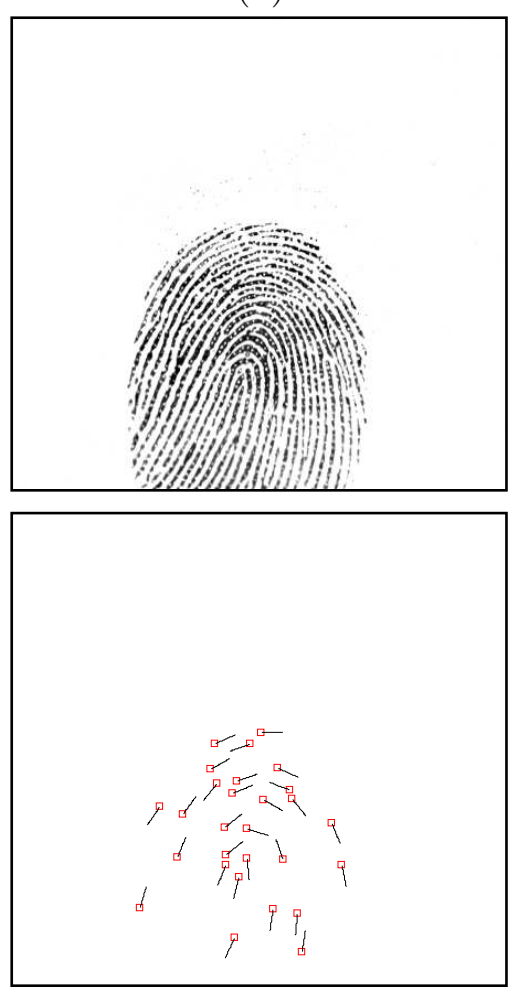

(d)

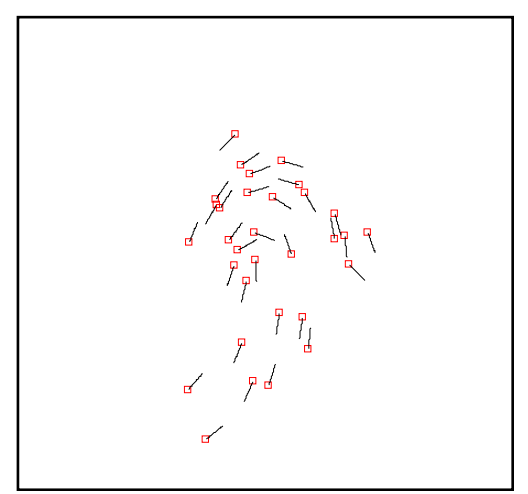

(b)
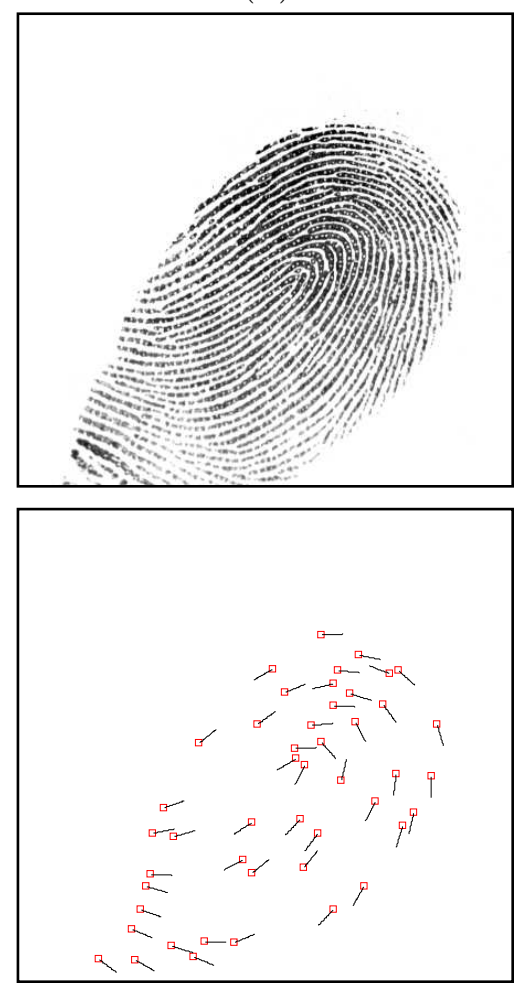

(e)

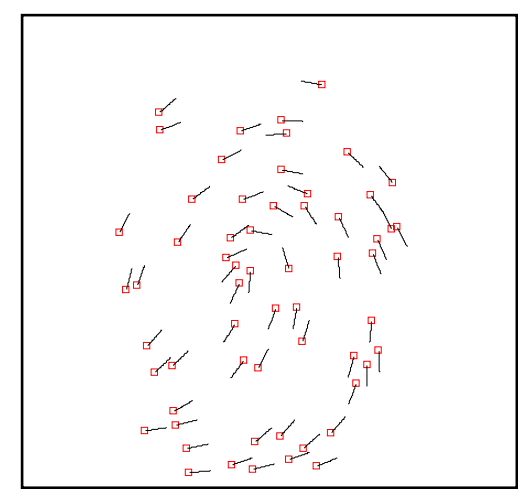

(c)
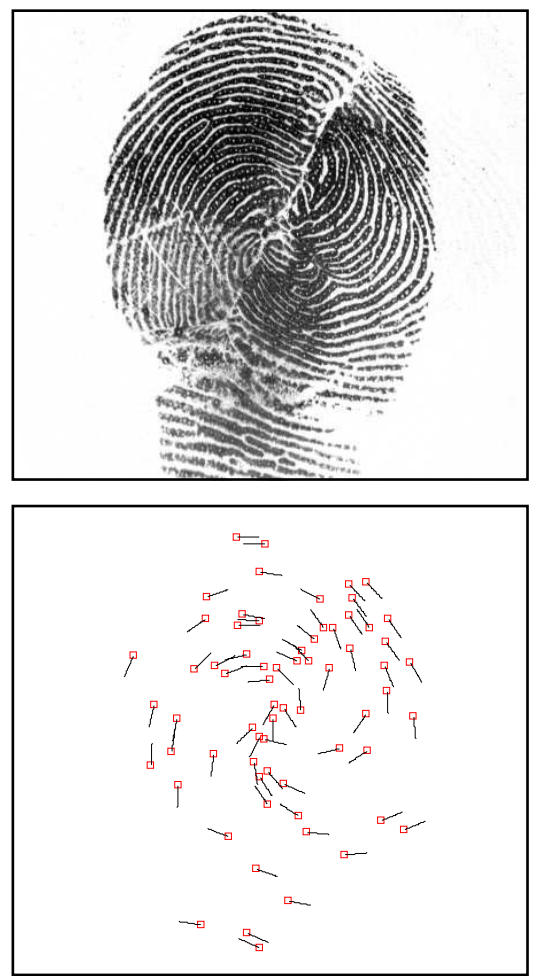

(f)

Figure 1.1: Fingerprint images with differing qualities and their corresponding extracted minutiae (a) Good quality image, (b) Dry image, (c) Smudged image, (d) Partial print, (e) Rotated image, (f) Image with scar/bruise. 


\subsubsection{Sensor Interoperability}

Historically, fingerprints have been acquired by smearing ink on the fingertip and creating an inked impression of the finger on paper. Advances in sensor technology now permit the online acquisition of fingerprints using scanners based on optical, capacitive, piezoelectric, thermal or ultrasonic principles [6, 11]. The sensing area of these scanners can vary from a few square millimeters to a few square inches. The resolution of the acquired image can vary between $250 \mathrm{dpi}$ (e.g., Authentec's AF-S2 FingerLoc) and 512 dpi (e.g., Digital Persona's U.are.U 4000); scanners that acquire 1000 dpi images of the fingerprint (e.g., Aprilis' HoloSensor) are also available in the market. The fingerprint images acquired using different sensing technologies can exhibit significant variations. This inherent variation in the acquired images is illustrated in Fig. 1.2, where five different scanners are used to capture impressions of the same fingerprint.
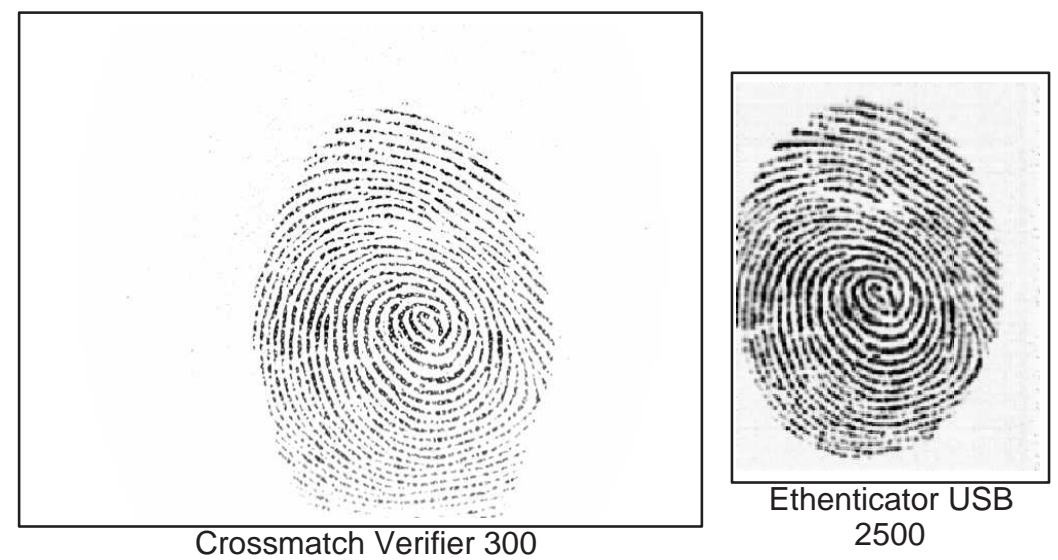

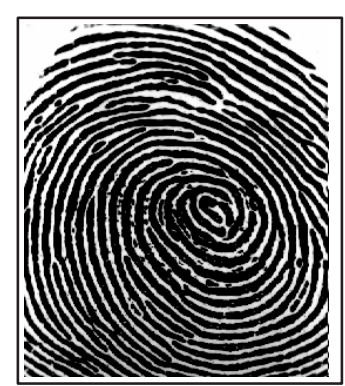

Secugen Hamster

III

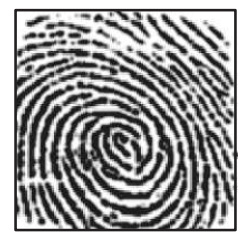

Precise 100AX

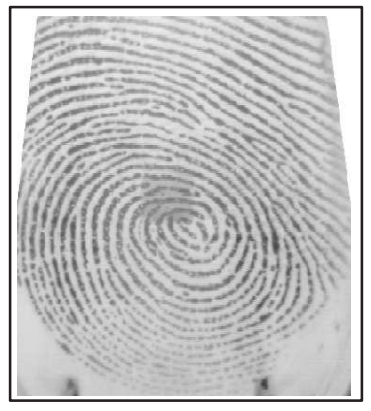

Digital Persona U.are.U 4000

Figure 1.2: Visual differences between impressions of the same finger acquired using five different sensors. Verifier 300, Hamster III and U.are.U 4000 are optical sensors. Hamster III is based on SEIR (Surface Enhanced Irregular Reflection) technology, while U.are.U 4000 and Verifier 300 use a FTIR (Frustrated Total Internal Reflection) technology. The USB 2500 is an electro-optical sensor and the 100AX is a capacitive sensor. 


\subsubsection{Quality}

Fingerprint quality is usually defined as a measure of the clarity of the ridge and valley structures, as well as the "extractability" of features (such as minutiae and singularity points) [10]. Also, in good quality images ridges and valleys flow smoothly in a locally constant direction [8]. If the ridge-valley pattern is prominent and clear then the fingerprint is said to be of good quality, whereas, if the captured ridge-valley pattern is not clear (contains some noise or irregularities) then the fingerprint is said to be of bad/poor quality.

\section{Factors affecting the quality of fingerprints}

There can be many factors which affect the quality of fingerprints.

(a) Excess or inadequate pressure

(b) Sweaty or dry fingers

(c) Temporary or permanent changes to the finger

(d) Sensor conditions

A poor quality fingerprint image captured by the sensor would lead to detection of false/spurious minutiae, loss of genuine minutiae and error in the position and orientation of the detected minutiae. Fig. 1.1 indicates the variations introduced in the extracted features due to images of poor quality.

\subsection{Motivation}

\subsubsection{Sensor Interoperability}

The variations introduced in the acquired images due to differences in resolution, scanning area, sensing technology, etc. impact the features extracted from the images (e.g., minutiae points) and, consequently, the templates stored in the database. Most fingerprint matchers are restricted in their ability to compare fingerprints originating from two different sensors resulting in poor inter-sensor performance [11]. Inferior inter-sensor performance has been reported not only in the fingerprint domain but in other domains such as speech, iris and face as well. Martin et al. [12] report a significant drop in the matching performance of speech-based biometric systems when the input device is switched from a carbon-button microphone to a electret microphone (and 
vice versa). Results of the iris recognition test conducted by the International Biometric Group (IBG) [13] suggest that inter-sensor matching performance is lower than intra-sensor performance. In the face domain, the variations introduced due to different cameras is expected to affect the performance of face recognition algorithms as severely as variations introduced due to differing illumination patterns [14]. A study conducted by Faltemier et. al. [15] reports a degradation in the matching performance of inter-sensor 3D face recognition. Their study concludes that intersensor 3D face matching may be facilitated under certain "limited" conditions by compensating for variations in the sampling densities, resolution accuracies and noise characteristics.

In order to motivate the problem of fingerprint sensor interoperability, we consider the USVISIT program that obtains fingerprint (and face) information of certain travelers arriving at the nation's airports and seaports. A 500 dpi optical scanner with a sensing area of 1.2 " $\times 1.2$ " is currently being used during the enrollment phase to procure fingerprint images. The introduction of a different sensor during the verification stage might render the current data unusable. The cost of re-enrolling individuals every time the sensor is changed will be tremendous. In applications like these, the need for sensor interoperability is paramount and will significantly impact the usability of the system.

\subsubsection{Quality}

Poor quality prints lead to improper feature detection. This would eventually lead to increasing FRR and FAR during recognition, and increasing FTE during enrollment. Thus, we need to assess fingerprint quality before storing the fingerprint template in the database. Also, the individual steps of the fingerprint recognition system can be adjusted on basis of the estimated quality [16].

The motivation for fingerprint quality estimation is derived from the increase in error rates of the best fingerprint matchers in FVC $2004^{3}$ in comparison with those of FVC $2002^{4}$ and FVC $2000^{5}$. The performance of FVC 2004 were found to be an order magnitude worse than that reported in earlier competitions (FVC 2002 and 2000) in terms of error rates [17]. FVC 2004 databases were marked by maximizing differences in finger placement, varying pressures and positions (vertical), along with exaggerated distortion and rotation effects. No efforts were made

\footnotetext{
${ }^{3}$ Fingerprint Verification Competition 2004 - http://bias.csr.unibo.it/fvc2004/default.asp

${ }^{4}$ Fingerprint Verification Competition 2002 - http://bias.csr.unibo.it/fvc2002/

${ }^{5}$ Fingerprint Verification Competition 2000 - http://bias.csr.unibo.it/fvc2000/
} 
to control image quality and the sensor platens were not systematically cleaned. Also, dry and wet variations were introduced in the captured fingerprint images.

\subsection{Contributions of this thesis}

In this thesis, two different problems - sensor interoperability and quality are addressed. A novel approach for fingerprint sensor interoperability is provided. In this work it is demonstrated that a simple non-linear calibration scheme, based on Thin Plate Splines (TPS), is sufficient to facilitate sensor interoperability in the context of fingerprints. In the proposed technique, the variation between the images acquired using two different sensors is modeled using nonlinear distortions. The proposed calibration model is tested on the MSU dataset comprising of fingerprint images obtained using two different sensor technologies: an optical Digital Biometrics (DBI) sensor and a solid-state capacitive VERIDICOM (VERI) sensor. Experiments indicate that the proposed calibration scheme improves the inter-sensor Genuine Accept Rate (GAR) by $\sim 35 \%$ to $\sim 40 \%$ at a False Accept Rate (FAR) of $0.01 \%$.

In terms of fingerprint quality, the incorporation of quality associated with each extracted feature into the match score formulation process is proposed. The feature quality is a measure of reliability and features with higher reliability should contribute more significantly than the less reliable features. The new quality-based score formulation is tested on two different kinds of matchers - a minutiae based matcher and a ridge feature-based matcher. Improvement in performance can be observed across multiple databases for both the matchers. With regards to fingerprint image quality, a new technique is proposed which classifies good, dry and smudged quality prints with a high degree of accuracy. The classification is based on features extracted from the fingerprint image response observed after applying a gabor filterbank.

\subsection{Organization}

In chapter 2, the intersensor distortion model developed to compensate for the intersensor variations is elaborated. The improved intersensor performance results are also presented. In chapter 3 , the new feature quality-based match score formulation is proposed and the gabor-based fingerprint image quality classification is also described. Finally in chapter 4, the approaches proposed for sensor interoperability and quality are summarized and the future work for each 
of the topics is presented. The Appendix comprises of a performance analysis of roll vs. plain fingerprints along with a few details of the fingerprint matchers utilized in this work. 


\section{Chapter 2}

\section{Sensor Interoperability}

\section{$2.1 \quad$ Introduction}

Fingerprints can be acquired using different methodologies. Based on the acquisition methodology, the fingerprint impression acquired from a fingertip may be categorized as being a rolled, dab, swipe or latent print (Fig. 2.1). Historically, the fingerprint impressions of an individual were imaged by rolling the inked finger surface on paper (Fig. 2.1(a)). Recently, advances in acquisition methodologies have led to the capture of dab (Fig. 2.1(b)) and swiped (Fig. 2.1(c)) fingerprints. Dab prints are obtained by simply pressing the finger against a flat surface (platen) of an electronic sensor. Swipe prints are obtained by swiping the finger against the scanner surface. Latent prints are prints which are lifted from crime scenes by a variety of techniques, including the use of chemicals, lasers, powders and other physical means (Fig. 2.1(d)).

Besides changes in sensing technology, the fingerprint acquisition and matching methodology may also vary across systems. Contact-based sensors can obtain rolled, flat, or slap prints of a finger (Fig. 2.2). The ability to successfully compare rolled prints against, for instance, the associated slap prints is indeed a challenging problem. This issue was highlighted in recent tests [1] involving FBI's IAFIS (that uses 10 rolled prints) and INS' IDENT system ${ }^{1}$ (that uses two flat prints). Interoperability between these types of prints may entail the adoption of new matching algorithms and/or indexing schemes. In this work we primarily concern ourselves with the interoperability between different sensing technologies (e.g., optical versus capacitive) and not between the modes of acquisition (e.g., rolled versus flat). A study of different modes of

\footnotetext{
${ }^{1}$ This is the fingerprint matching system used by DHS
} 


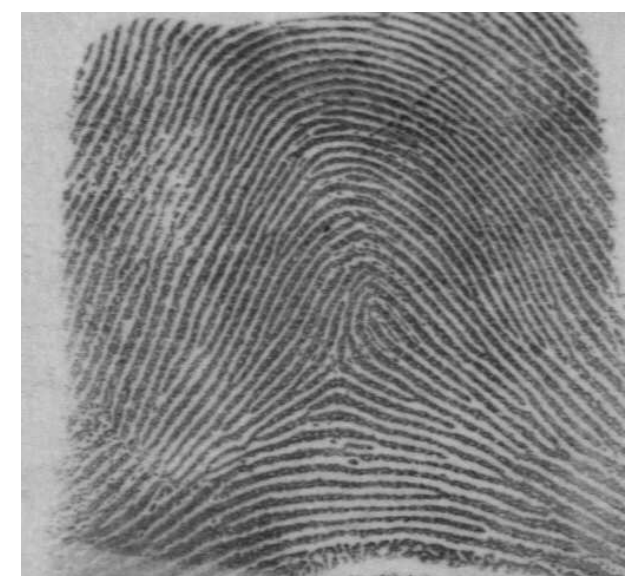

(a)

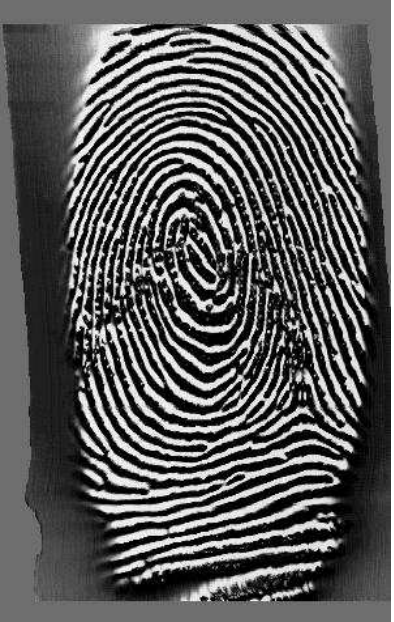

(c)

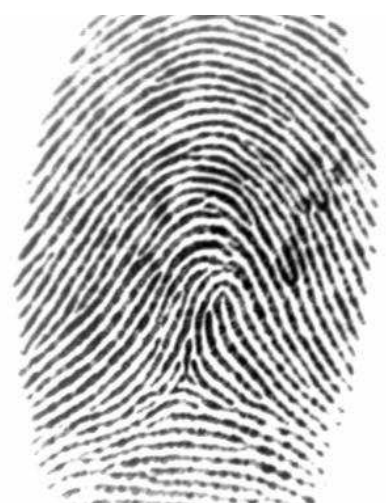

(b)

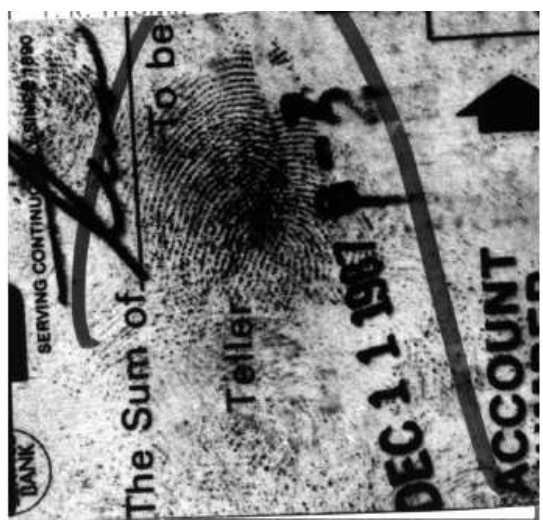

(d)

Figure 2.1: Four different methods for fingerprint acquisition. (a) Rolled print (from the NIST Special Database 4), (b) Dab print (from the FVC 2002 DB1 Database), (c) Swiped sensor print (from the FVC 2004 DB3 Database), (d) Latent print [1]

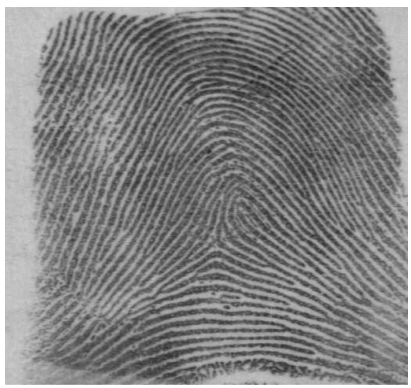

(a)

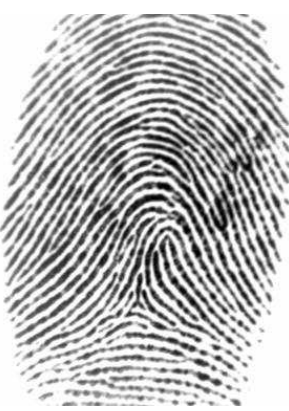

(b)

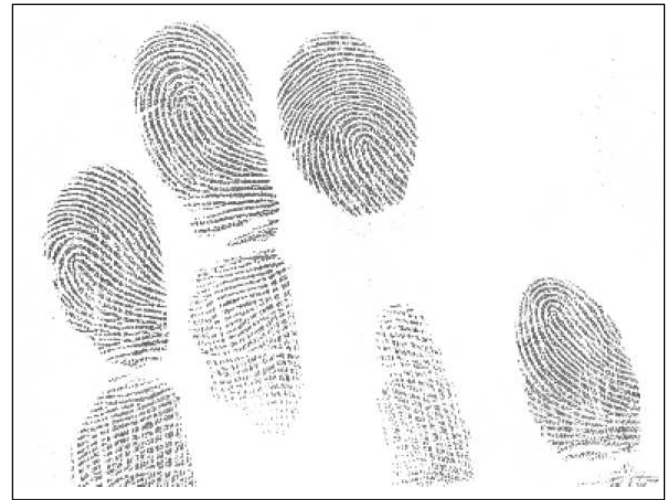

(c)

Figure 2.2: Fingerprint images obtained by different acquisition methodologies. (a) Rolled print (from the NIST Special Database 4), (b) Flat print (from the FVC 2002 DB1 Database), and (c) Slap print [2]. 
acquisition (rolled versus flat) is also conducted and a preliminary performance analysis report is provided in Appendix $\mathrm{A}$.

The problem of sensor interoperability, as defined in this work, cannot be solved by adopting a common biometric data exchange format [18]. Such a format merely aids in the exchange of feature sets between systems/vendors [19]. However, it does not provide a method to compare feature sets obtained from different sensors. Similarly, the Electronic Fingerprint Transmission Specification (EFTS) [20] defines requirements on fingerprint scanner systems and printers that supply fingerprint data to the Integrated Automated Fingerprint Identification System (IAFIS). The EFTS provides objective criteria for ensuring image quality whilst maintaining the geometric and spatial integrity of the supplied fingerprint images. However, it does not define a method to compare fingerprint images demonstrating significant differences in their physical dimensions.

In this work, we demonstrate that a simple non-linear calibration scheme is sufficient to facilitate sensor interoperability. In the proposed framework, the difference between the images acquired using two different sensors is modeled using non-linear distortions represented using Thin Plate Splines (TPS). The use of a non-linear distortion model that accounts for local warping is appropriate for the following reasons: (a) The physics of the sensing process can introduce distortions unique to each sensor technology. Even a versatile matcher may fail to account for such types of distortions. (b) The nature of the distortion may vary across the sensor due to the arrangement of the sensing elements within the device; thus, a linear global transformation may be unsuitable.

The remainder of the chapter is organized as follows. In Section 2.2 some of the commonly encountered fingerprint sensing technologies are enumerated; Section 2.3 discusses two possible approaches to reconcile images originating from multiple sensors; Section 2.4 presents the average deformation model used in the proposed approach to model inter-sensor distortions; Section 2.5 discusses experimental results on the MSU database suggesting the efficacy and relevance of the proposed scheme; Section 2.6 concludes this chapter with a discussion on the merits and demerits of our approach. 


\section{$2.2 \quad$ Fingerprint Sensing Technology}

In this section, the working principle of different sensing technologies are described. The sensing technologies have been categorized as optical (FTIR and SEIR), capacitive, piezoelectric, temperature differential, ultrasound, touchless and multispectral (see Table 2.1).

\subsubsection{Optical}

Optical sensors based on Frustrated Total Internal Reflection (FTIR) consist of a glass prism, a light source and a focusing lens along with a CMOS or a CCD camera. The light is directed onto the surface of the prism, which acts as a platen for the finger to be placed, and the reflected light is focused by the lens onto the camera which captures the fingerprint image (Fig. 2.3(a)). Light rays are totally reflected from the valleys, giving them the lighter appearance, while light rays are not reflected from the ridges and hence the ridges appear darker in the captured image. The focal length of the lens is a deciding factor in the size of the sensor. If a lens having small focal length is selected, optical distortion could be observed in the captured images. Hence, a trade-off between the sensor size and optical distortion has to be obtained. The optical sensors are normally bulky because of this set-up.

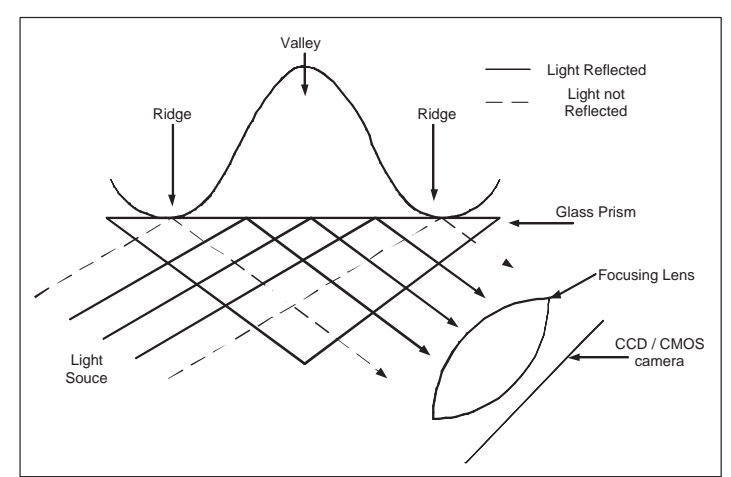

(a) Optical

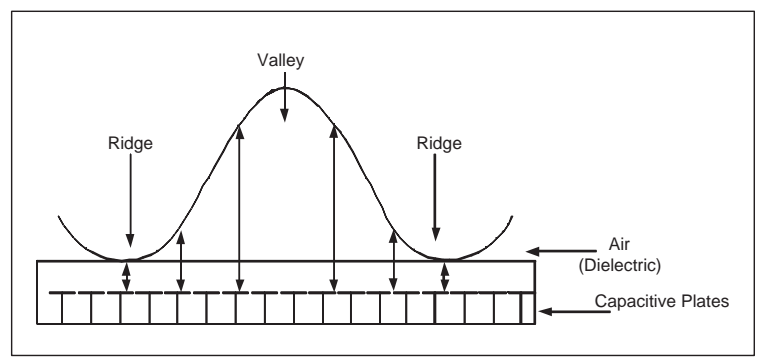

(b) Capacitive

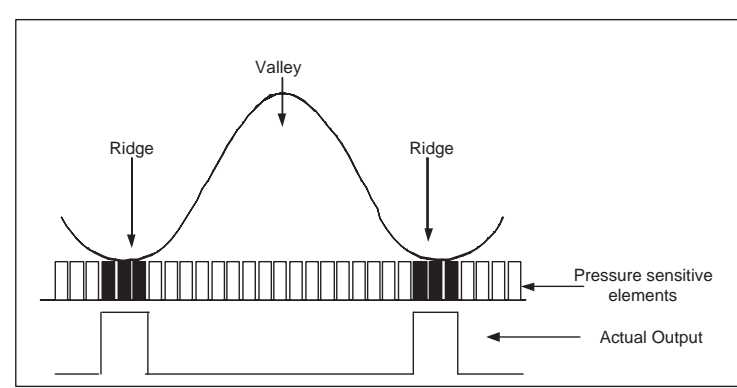

(c) Piezoelectric

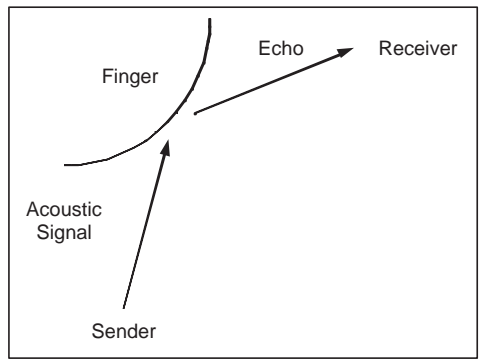

(d) Ultrasound

Figure 2.3: Working principles of different sensing technologies 
Table 2.1: Fingerprint Sensing Technologies.

\begin{tabular}{|c|c|c|c|}
\hline No. & $\begin{array}{l}\text { Sensing } \\
\text { Technology }\end{array}$ & Working Principle & Products \\
\hline 1 & $\begin{array}{l}\text { Optical } \\
\text { (FTIR - } \\
\text { Frustrated } \\
\text { Total Internal } \\
\text { Reflection) }\end{array}$ & $\begin{array}{l}\text { FTIR is based on total internal reflection. } \\
\text { Light is reflected from valleys and not from } \\
\text { ridges. Lens focuses the reflected light } \\
\text { rays onto the camera. Valleys appear } \\
\text { bright while ridges appear dark. } 11,21\end{array}$ & $\begin{array}{l}\text { Digital Persona } \\
\text { U.are.U } 4000\end{array}$ \\
\hline 2 & $\begin{array}{l}\text { Optical } \\
\text { (SEIR - Surface } \\
\text { Enhanced } \\
\text { Irregular } \\
\text { Reflection) }\end{array}$ & $\begin{array}{l}\text { SEIR is based on scattering principles. } \\
\text { Light is reflected and scattered from ridges } \\
\text { and not from valleys. Most of the scattered } \\
\text { light is collected, hence ridges appear } \\
\text { bright while valleys appear dark. [21] }\end{array}$ & $\begin{array}{l}\text { Secugen } \\
\text { Hamster III }\end{array}$ \\
\hline 3 & Capacitive & $\begin{array}{l}\text { Air acts as dielectric medium. Capacitance } \\
\text { is a function of the distance of ridges and } \\
\text { valleys from the capacitive plates. [11] }\end{array}$ & $\begin{array}{l}\text { Fujitsu MBF200 } \\
\text { Precise 100AX }\end{array}$ \\
\hline 4 & Piezoelectric & $\begin{array}{l}\text { Current is generated as a function of varying } \\
\text { pressure applied by ridges and valleys } \\
\text { on a dielectric material. [11 }\end{array}$ & $\begin{array}{l}\text { Fidelica } \\
\text { Microsystems } \\
\text { FIS } 3002\end{array}$ \\
\hline 5 & $\begin{array}{l}\text { Temperature } \\
\text { Differential }\end{array}$ & $\begin{array}{l}\text { Current is generated as a function of varying } \\
\text { temperature differentials observed across } \\
\text { ridges and valleys. [11] }\end{array}$ & $\begin{array}{l}\text { Atmel } \\
\text { AT77C101B } \\
\text { Swipe sensor }\end{array}$ \\
\hline 6 & Ultrasound & $\begin{array}{l}\text { Image generated on basis of the response } \\
\text { of the acoustic wave bounced off the } \\
\text { fingertip. No skin contact. [11] }\end{array}$ & $\begin{array}{l}\text { Ultra-Scan } \\
\text { UltraTouch } \\
\text { Model } 203\end{array}$ \\
\hline \multirow[t]{2}{*}{7} & \multirow[t]{2}{*}{ Touchless } & $\begin{array}{l}\text { 3-D image generated by integrating images } \\
\text { captured by different cameras. }\end{array}$ & $\begin{array}{l}\text { TBS 3-D sensor. } \\
\text { Surround Imaging }(\mathrm{tm}) \\
\text { technology }\end{array}$ \\
\hline & & $\begin{array}{l}\text { Light reflected by ridges is converted into } \\
\text { electrical signals to generate a image. } \\
\text { Contact-less. }\end{array}$ & $\begin{array}{l}\text { TST group } \\
\text { BiRDIIi }\end{array}$ \\
\hline 8 & Multispectral & $\begin{array}{l}\text { Multispectral data is collected under } \\
\text { different illumination angles and polarizing } \\
\text { conditions as well as different wavelengths. } \\
\text { Fingerprint information below the surface } \\
\text { of the skin is captured easily. [22] }\end{array}$ & $\begin{array}{l}\text { Lumidigm } \\
\text { LightPrint } \\
\text { Technology }\end{array}$ \\
\hline
\end{tabular}


Some optical sensors use a Surface Enhanced Irregular Reflection (SEIR) technique to capture fingerprints. SEIR is based on scattering principles in which light is scattered and reflected from ridges and not from valleys. Most of the scattered light is collected, giving the ridges a brighter appearance and the valleys a darker appearance.

\subsubsection{Capacitive}

This technique consists of a number of small capacitive plates placed under the sensor platen. Air acts as the dielectric medium and the electric field strength is a function of the distance of the fingerprint ridges and valleys from the capacitive plates (Fig. 2.3(b)). The electric field drops off as the inverse of the distance [23]. To achieve a certain resolution, a compromise between the capacitive plate size and the distance between capacitive plates needs to be obtained. Capacitive sensors suffer from static discharge which could possibly damage the plates; hence proper grounding is required.

\subsubsection{Piezoelectric}

The surface of the pressure sensitive sensors is made up of a dielectric material which generates current proportionate to the applied pressure. Ridges touching the sensor surface apply more pressure as compared to the valleys not touching the sensor surface [11]. This varying current generated due to varying pressures aids in capturing the fingerprint image (Fig. 2.3(c)).

\subsubsection{Temperature Differential}

Sensors based on this technique use pyroelectric material which generates current proportional to temperature differentials [11]. The fingerprint ridges in contact with the sensor generate a temperature differential slightly different from the valleys not touching the platen. These sensors are electrically heated to maintain a predetermined temperature. The working principle is same as that of the piezoelectric device, only the pressure sensing elements are replaced by temperature sensitive elements. 


\subsubsection{Ultrasound}

These sensors transmit small pulses of acoustic signals towards the finger and pick up the reflected echo's. The sensors are however bulky and expensive as they consist of a sender which transmits the acoustic signal, and a receiver which detects the responses bouncing off the fingertip [11. Ultrasound sensors have a significant advantage over other sensors as they work on a noncontact principle eliminating the distortion introduced due to surface contact (Fig. 2.3(d)).

\subsubsection{Touchless}

Recently a touchless fingerprint sensor was manufactured by TBS North America ${ }^{2}$. They combine images captured using a touchless multi-sensor device to generate a 3-D model of the fingerprint. During the acquisition process, the finger is illuminated by a sequence of green LED array combinations, generating 20 images (4 images/camera x 5 cameras). The 20 images are calibrated appropriately to construct a 3-D fingerprint image. To ensure compatibility with the traditional databases and algorithms, the 3-D finger is virtually unrolled onto a plane to obtain a rolled-equivalent fingerprint. This sensor helps in nullifying the distortions introduced during the image acquisition process, due to interaction of elastic skin with a solid surface. Also, a sensor developed by the TST group ${ }^{3}$ converts light reflected by ridges into electrical signals to generate an image.

\subsubsection{Multispectral}

A recent sensing technology proposed by Lumidigm ${ }^{4}$, images sub-surface information from fingerprints. The sub-surface information is believed to mimic the ridge-valley skin pattern [22]. The multispectral data is collected using different illumination angles, polarization conditions and different wavelengths. The advantage of using multispectral imaging is that good quality prints are acquired irrespective of differing skin conditions and environmental effects. Dry/smudged fingers or fingers with scars are still captured properly as these effects do not affect the subsurface information.

\footnotetext{
${ }^{2}$ http://www.tbsinc.com/

${ }^{3}$ http://www.tst-ag.de/

${ }^{4}$ http://www . lumidgim.com/
} 


\subsubsection{Distortions introduced in different sensing technologies}

Each sensing technology introduces its own distortions. Thus, deformation in fingerprint images is not only a consequence of the elastic skin interacting with a solid platen/surface during the image acquisition process, but a characteristic of the sensor as well. Distortions can occur in a fingerprint image when parts of the image are stretched, compressed or out of focus with respect to the rest of the image. Blurred edges are sometimes observed in images captured using optical scanners. This is due to the relatively larger size of the fingerprint area compared to the first lens in the lens assembly, leading to non-parallel light paths toward the edge of the image [21]. In some acquisition systems, the path lengths of reflected light differ across the length and width of the fingertip. Differences in path lengths can cause part of the image to be wider than the rest of the image - a principle known as Trapezoidal Distortion [21], 24], [25]. In Fig. 1.2, the image captured using Digital Persona's U.are.U 4000 exhibits this type of distortion. Varying path lengths also generate defocused areas within the captured image. The curvature of the lens assembly can lead to curved or out of focus appearance along the outer edges of the image. Capacitive sensors are prone to noisy artifacts, including noise from the $60 \mathrm{~Hz}$ power line and electrical noise from within the sensor. The semiconductor-sensing chips are also sensitive to electrostatic discharge, salt from sweat and other contaminants, as well as physical wear. Grid artifacts are possible in capacitive sensors. Hence, intrinsic sensor properties introduce distortions in the resulting images.

\subsection{Sensor Interoperability}

Biometric sensor interoperability refers to the ability of a system to compensate for the variability introduced in the raw biometric data of an individual due to the deployment of different sensors. In the context of fingerprints, interoperability may be addressed by two different approaches as shown in Fig. 2.4.

(a) Distortion compensation model: In this approach, the goal would be to determine and model the physics of the distortion process when a user places her finger on a particular scanner. This distortion would be based on the sensing technology of a particular scanner as well as the process employed to convert the sensed data into a raw image. As shown in Fig. 2.4(a), knowledge of the distortion process will permit computation of the original "undistorted" 
fingerprint (canonical image). The canonical image may then be used for matching purposes [26]. (b) Inter-sensor distortion model: In the second approach, the relative distortion between images acquired using two different sensors can be computed (Fig. 2.4(b)). Modeling the intersensor distortion may be viewed as a calibration problem, and can be accomplished by inheriting the knowledge of corresponding points on the two sensors.

This is similar to the camera calibration problem in computer vision wherein the knowledge of corresponding points on a chessboard plane is used for appropriately registering two different cameras as well as computing the intrinsic parameters for the same camera [27], [28], [29], [30]. As multiple inexpensive cameras are used in several close-range 3-D and 2-D measurement applications, precise calibration of cameras is required [29]. Camera calibration in the context of 3-D machine vision is the process of determining the internal camera geometric and optical characteristics (intrinsic parameters) and/or the 3-D position and orientation of the camera frame relative to a certain world coordinate system (extrinsic parameters) [30]. Extrinsic parameters are required to transform object coordinates to a camera-centered coordinate frame while the intrinsic parameters include the effective focal length, scale factor and image center/principal point [30].

The inter-sensor distortion model is similar to camera calibration and is a combination of affine as well as elastic distortions.

In this work, the second approach described above is used to address interoperability. A thin-plate spline (TPS) model is used to represent the inter-sensor distortion since such a model can account for the affine as well as the non-linear aspects of the deformation. The parameters of the inter-sensor distortion model rely on the evidence of control points present on the two sensors and their correspondence. In the proposed approach, corresponding points (control points) are obtained by manually locating minutiae points from a small set of representative fingerprint image pairs (Fig. 2.5). The control points are selected manually to approximately cover the whole area within the smaller fingerprint image in order to model the distortions occurring in different areas of the representative image. These control points are then used to derive a deformation that represents the relative distortion between the two images (Fig. 2.6).

Procrustes analysis of the control points assists in the computation (and subsequent removal) of translation and rotational parameters relating the two representative images. Procrustes analysis is a rigid shape analysis that uses isomorphic scaling, translation, and rotation 


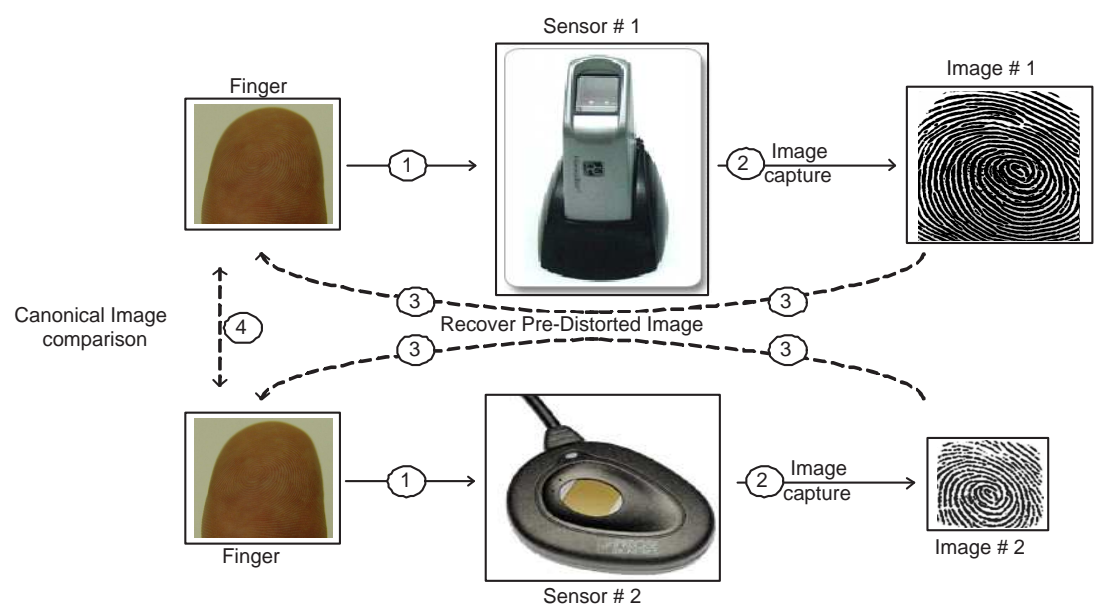

(a)

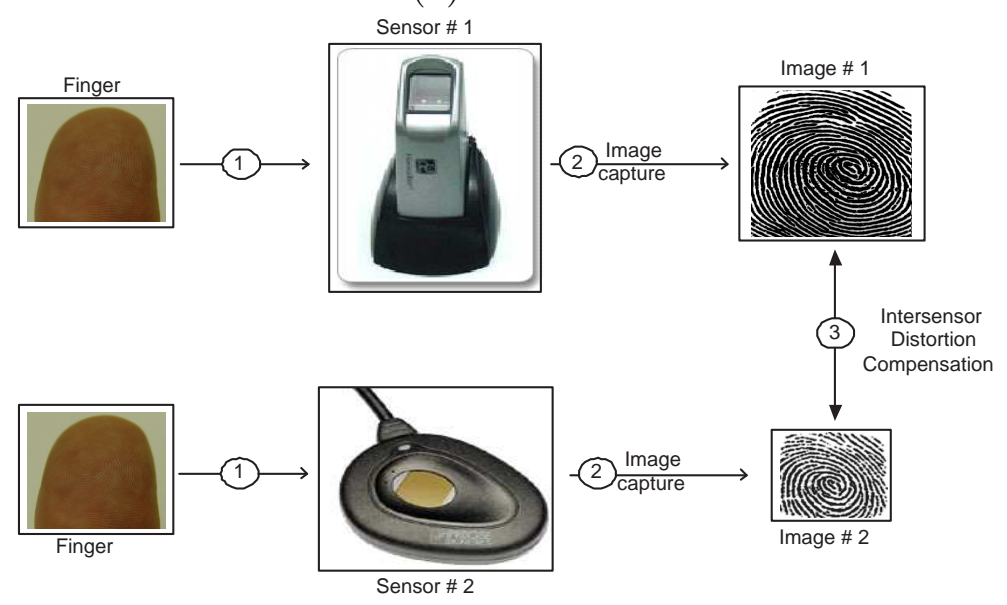

(b)

Figure 2.4: Two different ways of facilitating interoperability. (a) Distortion compensation model and (b) Inter-sensor distortion model. The numbers within circles denote the sequence of steps.

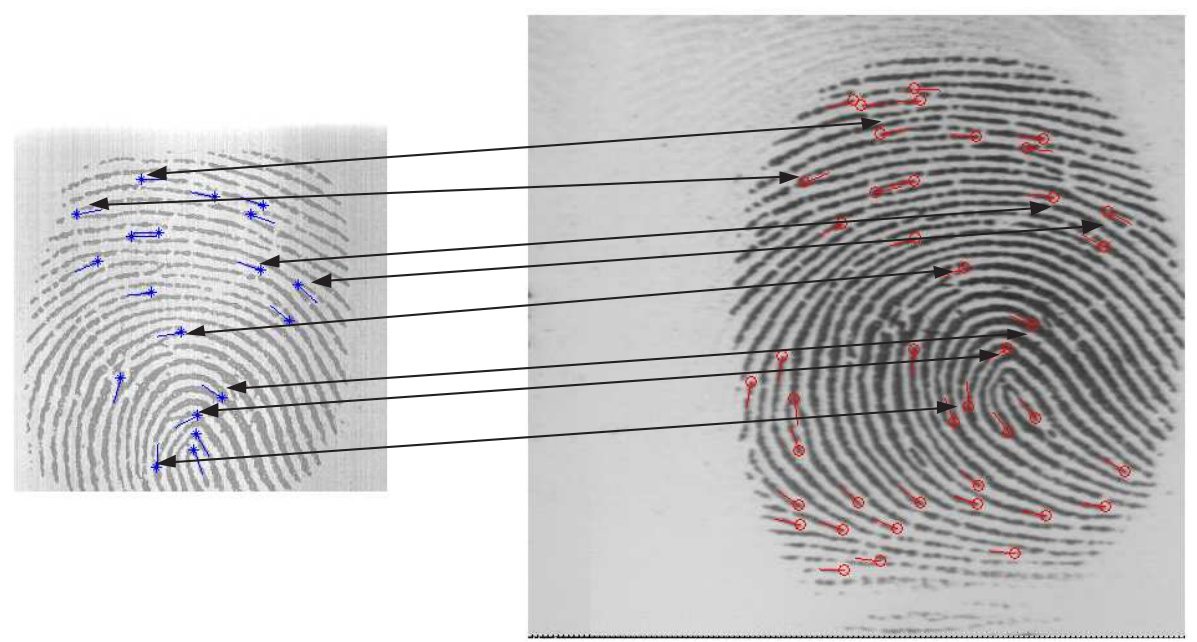

Figure 2.5: Minutiae correspondence (manually selected) across representative image pairs, serve as inputs to the TPS model. 


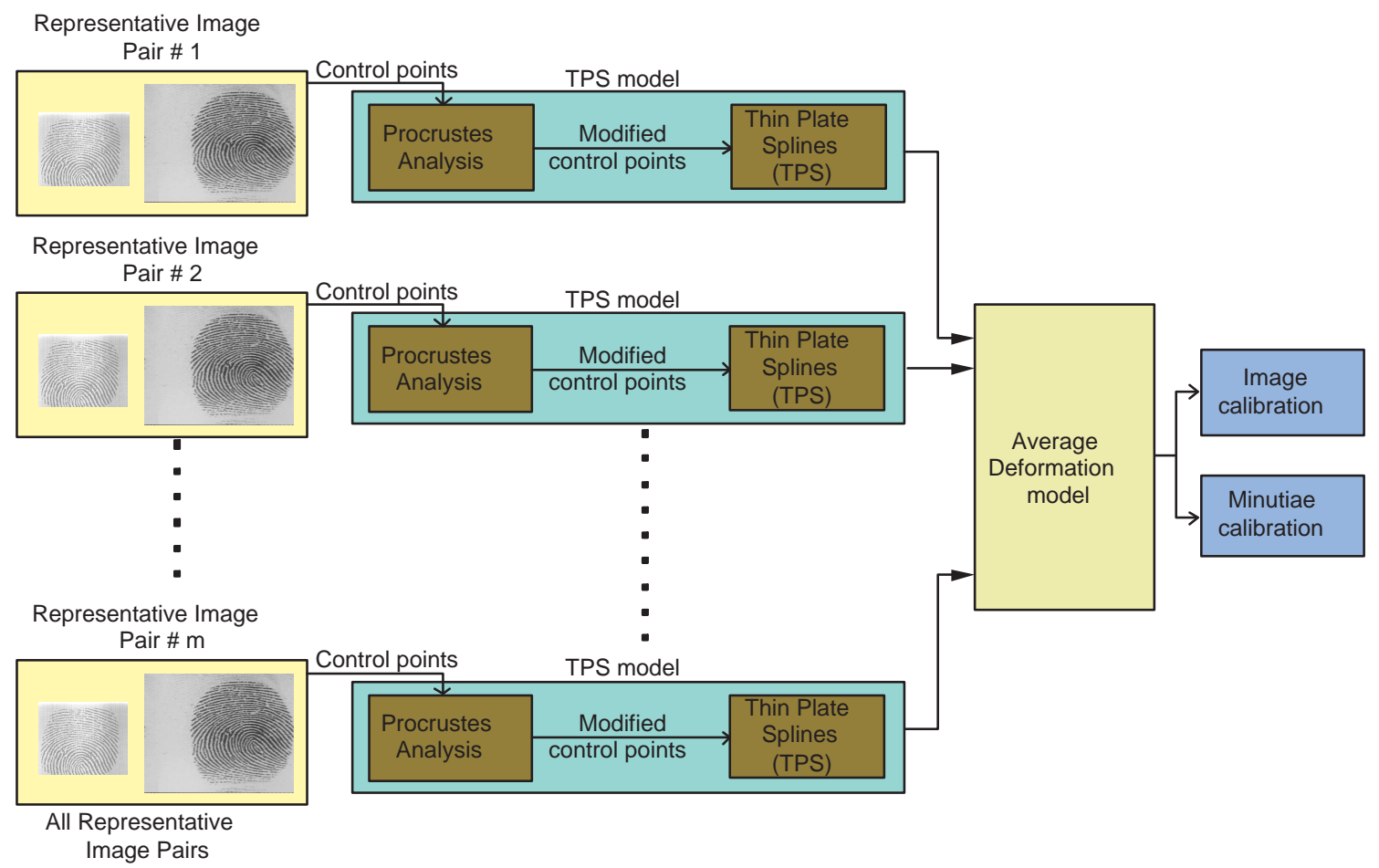

Figure 2.6: Manually selected control points from representative image pairs are provided as inputs to the TPS model. The affine and non-linear parameters derived from the average deformation model are used for image and minutiae calibration during the authentication stage.

to find the "best" fit between two landmarked shapes defined by the control points. In our methodology, procrustes analysis is used to determine only the translation and rotation parameters. The translation parameters are obtained by aligning the centroids of both sets of control points. If $P=\left\{\left(u_{1}, v_{1}\right),\left(u_{2}, v_{2}\right), \cdots,\left(u_{n}, v_{n}\right)\right\}$ is the set of $n$ control points in sensor 1 and $P^{\prime}=\left\{\left(u_{1}^{\prime}, v_{1}^{\prime}\right),\left(u_{2}^{\prime}, v_{2}^{\prime}\right), \cdots,\left(u_{n}^{\prime}, v_{n}^{\prime}\right)\right\}$ is the corresponding set of $n$ control points in sensor 2 , then the centroids are given by $\left(\bar{u}=\sum_{i=1}^{n} \frac{u_{i}}{n}, \bar{v}=\sum_{i=1}^{n} \frac{v_{i}}{n}\right)$ and $\left(\bar{u}^{\prime}=\sum_{i=1}^{n} \frac{u_{i}^{\prime}}{n}, \bar{v}^{\prime}=\sum_{i=1}^{n} \frac{v_{i}^{\prime}}{n}\right)$, respectively. The control points are next zero-centered based on their centroids. The zero-centered $P$ and $P^{\prime}$ are symbolized as $P_{M}$ and $P_{M}^{\prime}$. The optimal rotation between the two point clouds is computed by estimating the orthogonal matrix $Q$ such that the expression $m=\left\|P_{M}-Q P_{M}^{\prime}\right\|$ is minimized. In order to maximize the correlation between the two sets of landmark points, the optimal $Q$ is calculated by the singular value decomposition (SVD) of $P_{M}^{T} P_{M}^{\prime}$, i.e., SVD $\left(P_{M}^{T} P_{M}^{\prime}\right) \rightarrow U D V^{T}$, and $Q=V U^{T}$

The rotation and translation factors are due to variation in finger placement across different acquisitions and do not contribute toward the intersensor distortions. The TPS model, described in the next section, is used to compute the affine and the non-linear parameters from 
the translation/rotation-corrected control points. By first compensating for the translation and rotation parameters, the TPS deformation parameters can be computed independently of these effects. The TPS parameters are estimated for several representative image pairs based on manually established control points. Multiple sets of TPS parameters aid in generating an average deformation model [31, which defines the perturbation at every pixel (point) on one sensor with respect to the other.

The resulting average deformation model may be used for minutiae as well as image calibration. Our proposed inter-sensor distortion model can be incorporated into a minutiae-based matcher for successful comparison of templates originating from different sensors as shown in Fig. 2.7. In image calibration, the original fingerprint image is subjected to the transformation

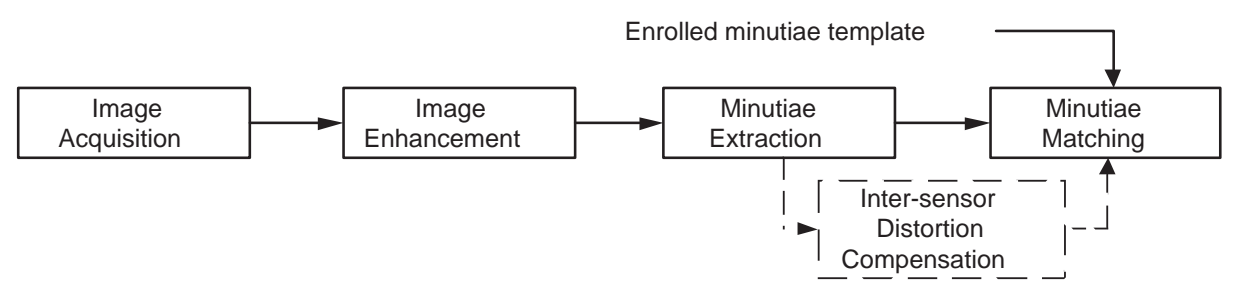

Figure 2.7: Minutiae calibration: The inter-sensor distortion compensation block is introduced in-between the minutiae extraction and matching modules to handle inter-sensor distortions.

defined by the average deformation. Minutiae are then extracted from this calibrated image and later utilized during the matching stage.

\subsection{Thin Plate Spline model}

For spatial rearrangement of points, the thin-plate spline succinctly expresses the dependence of the physical bending energy of a thin metal plate on point constraints [32]. TPS has been used in many 2D [33], [34], [35], [36] and 3D [34], [37], [35], 36] medical imaging applications for appropriate registration. Recently, TPS has been used to model the non-linear deformations in fingerprints [31], [9], [38], [39], [40], [41].

\subsubsection{Bending Energy of TPS}

Given a list of corresponding points, the TPS model interpolates the corresponding grid points while maintaining smoothness as defined by the bending energy of the thin metal plate. 
The smoothness is maintained by minimizing the bending energy at a point ,

$$
\iint_{R^{2}}\left(\left(\frac{\partial^{2} F_{k}}{\partial x^{2}}\right)^{2}+2\left(\frac{\partial^{2} F_{k}}{\partial x \partial y}\right)^{2}+\left(\frac{\partial^{2} F_{k}}{\partial y^{2}}\right)^{2}\right) d x d y
$$

where, $F_{k}$ can be defined in the $(x, y)$ plane (as an inplane interpolant) or even orthogonal to the $(x, y)$ plane.

The following derivation is based on Bookstein [32]. Let $G_{1}=\left(u_{1}, v_{1}\right), G_{2}=\left(u_{2}, v_{2}\right), \cdots$, $G_{n}=\left(u_{n}, v_{n}\right)$ be the control points in sensor 1 and $G_{1}^{\prime}=\left(u_{1}^{\prime}, v_{1}^{\prime}\right), G_{2}^{\prime}=\left(u_{2}^{\prime}, v_{2}^{\prime}\right), \cdots, G_{n}^{\prime}=\left(u_{n}^{\prime}, v_{n}^{\prime}\right)$ be the corresponding control points in sensor 2. The basis function is given by $U\left(r_{i j}\right)=r_{i j}^{2} \log \left(r_{i j}^{2}\right)$, where $r_{i j}=\left|G_{i}-G_{j}\right|$ and $U(r)$ satisfies the equation for minimizing the bending energy at a point. Then matrices $K$ and $P$ can be defined as,

$$
\mathbf{K}=\left[\begin{array}{cllr}
0 & U\left(r_{12}\right) & \cdots & U\left(r_{1 n}\right) \\
U\left(r_{21}\right) & 0 & \cdots & U\left(r_{2 n}\right) \\
\cdots & \cdots & \cdots & \cdots \\
U\left(r_{n 1}\right) & U\left(r_{n 2}\right) & \cdots & 0
\end{array}\right]_{n \times n}
$$

and

$$
\mathbf{P}=\left[\begin{array}{ccc}
1 & u_{1} & v_{1} \\
1 & u_{2} & v_{2} \\
\cdots & \cdots & \cdots \\
1 & u_{n} & v_{n}
\end{array}\right]_{n \times 3}
$$

Let ${ }^{T}$ be the matrix transpose operator and $O$ be a $3 \times 3$ matrix of zeros, then $L$ is given as

$$
\mathbf{L}=\left[\begin{array}{c|c}
K & P \\
\hline P^{T} & O
\end{array}\right]_{(n+3) \times(n+3)} .
$$

Define $Y$ as

$$
\mathbf{Y}=\left[\begin{array}{llll|lll}
u_{1}^{\prime} & u_{2}^{\prime} & \cdots & u_{n}^{\prime} & 0 & 0 & 0 \\
v_{1}^{\prime} & v_{2}^{\prime} & \cdots & v_{n}^{\prime} & 0 & 0 & 0
\end{array}\right]_{2 \times(n+3)}
$$

The affine and non-linear deformation parameters can be estimated as,

$$
L^{-1} Y^{T}=(W \mid h)^{T}
$$

where,

$$
\mathbf{W}=\left[\begin{array}{llll}
w_{1 u} & w_{2 u} & \cdots & w_{n u} \\
w_{1 v} & w_{2 v} & \cdots & w_{n v}
\end{array}\right]_{2 \times n} ; \quad h=\left[\begin{array}{lll}
h_{1 u} & h_{2 u} & h_{2 u} \\
h_{1 v} & h_{2 v} & h_{2 v}
\end{array}\right]_{2 \times 3}
$$


$h_{1}$ 's, $h_{2}$ 's, $h_{3}$ 's are the parameters of the affine transformation while $w_{i}$ 's represent the weights for the non-linear transformation.

If a list of corresponding points between the $k^{\text {th }}$ representative image pair is defined, the TPS function, $F_{k}$, calculates the transformed co-ordinates $\left(x^{\prime}, y^{\prime}\right)$ as a function of the original coordinates $(x, y)$. Here, the $(x, y)$ points are in sensor 1 and the $\left(x^{\prime}, y^{\prime}\right)$ points are the corresponding transformed points in sensor 1 . The function $F_{k}$ is defined for each pixel $(x, y)$ on sensor 1 and can be written as

$$
\left(x^{\prime}, y^{\prime}\right)=F_{k}(x, y)=\left[\begin{array}{l}
h_{1 u} \\
h_{1 v}
\end{array}\right]+\left[\begin{array}{l}
h_{2 u} \\
h_{2 v}
\end{array}\right] x+\left[\begin{array}{l}
h_{3 u} \\
h_{3 v}
\end{array}\right] y+\sum_{i=1}^{n}\left[\begin{array}{l}
w_{i u} \\
w_{i v}
\end{array}\right] U\left(\left|G_{i}-(x, y)\right|\right),
$$

where $, G_{i}, i=1$..n, are the control points corresponding to sensor 1 . The affine parameters and the non-linear weights can be derived in a closed form solution according to the bending energy constraint which minimizes the curvature at every point in the grid.

\subsubsection{Determining the average pixel deformation}

If there are $m$ representative image pairs, then the application of the procedure described above will result in the generation of $m$ TPS functions, $F_{1}, F_{2}, \ldots F_{m}$. The average deformation of an arbitrary pixel $(x, y)$ on sensor 1 , denoted by $\bar{F}(x, y)$, is computed from these $m$ functions as,

$$
\bar{F}(x, y)=\sum_{k=1}^{m} \frac{F_{k}(x, y)}{m} .
$$

The function $\bar{F}(x, y)$ defines the new location of each point $(x, y)$ in sensor 1 .

\subsubsection{Orientation computation}

The orientation of each minutiae has to be modified after estimating the average deformation (Fig. 2.8). This is accomplished by the procedure described below.

Let $P(a, b)$ be the location of a minutia and $\theta$ its orientation. The "angle point" $Q(c, d)$ can then be computed at a distance $r$ from $P$ as,

$$
c=a+r \cos \theta, \quad d=b+r \sin \theta .
$$

Upon applying the average deformation model, the points $P(a, b)$ and $Q(c, d)$ are altered as $P^{\prime}\left(a^{\prime}, b^{\prime}\right)$ and $Q^{\prime}\left(c^{\prime}, d^{\prime}\right)$ respectively. The new orientation $\theta^{\prime}$ can now be computed as,

$$
\tan \theta^{\prime}=\frac{d^{\prime}-b^{\prime}}{c^{\prime}-a^{\prime}}
$$




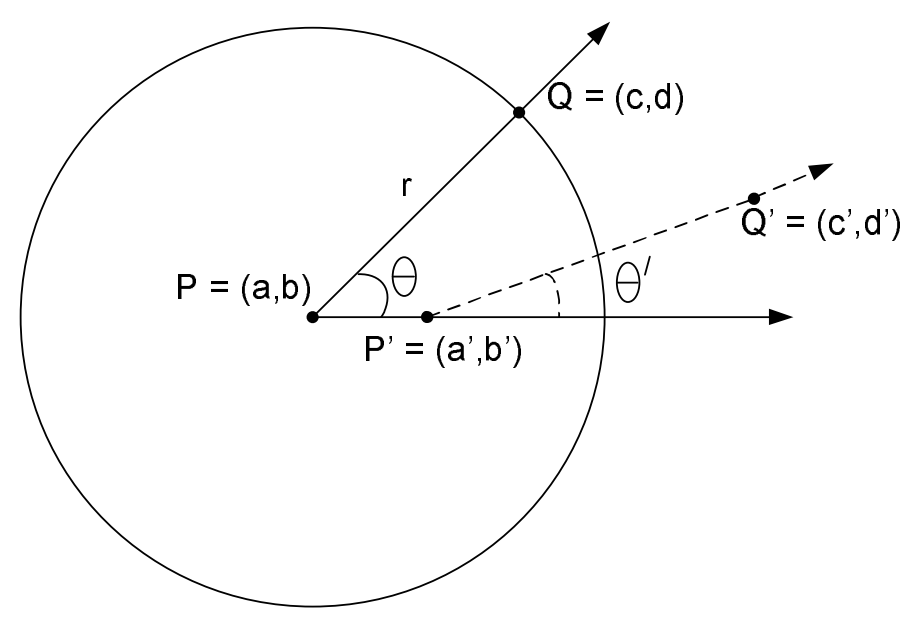

Figure 2.8: Minutiae orientation is modified on basis of the transformed minutiae location (after average deformation). $\mathrm{P}$ is the original minutiae point location while $\mathrm{Q}$ is the corresponding 'angle point'. $\mathrm{P}$ ' and Q' are the corresponding locations of $\mathrm{P}$ and $\mathrm{Q}$ after average deformation.

\subsubsection{Derivation of scaling parameters}

The linear terms obtained from TPS are collected in a matrix,

$$
\mathbf{H}=\left[\begin{array}{ll}
h_{2 u} & h_{3 u} \\
h_{2 v} & h_{3 v}
\end{array}\right] .
$$

The $h_{1 u}$ and $h_{1 v}$ terms can be ignored as they are the translation parameters. Singular decomposition of $H$ results in $H=O_{1} D O_{2}$, where $O_{1}$ and $O_{2}$ are the rotation parameters and $D$ is a diagonal matrix of singular values that correspond to the scaling parameters in the horizontal $S_{u}$ and vertical $S_{v}$ directions. The optimal scaling parameters $S_{U}, S_{V}$ are obtained by averaging the scaling parameters across $k$ different pairs of representative images (Eq. 2.6).

$$
S_{U}=\sum_{k=1}^{m} \frac{S_{u_{k}}}{m} ; \quad S_{V}=\sum_{k=1}^{m} \frac{S_{v_{k}}}{m}
$$

\subsection{Experimental Results}

In order to test the efficacy of the proposed calibration model, the MSU dataset comprising of fingerprint images obtained using two different sensor technologies, an optical Digital Biometrics (DBI) sensor and a solid-state capacitive VERIDICOM (VERI) sensor [42], was used. The 500 dpi DBI sensor has a platen area of $1 " \times 1 "$ and outputs images of size $480 \times 508$. The 500 dpi VERI sensor has a sensing area of $0.6 " \times 0.6 "$ and outputs images of size $300 \times 300$. Sample images from each sensor can be seen in Fig. 2.5. We observe that the two images, although 


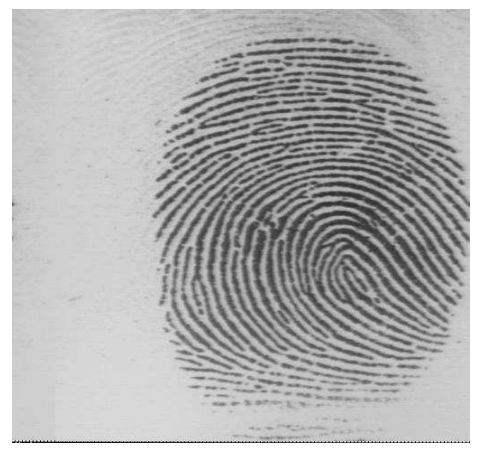

(a)

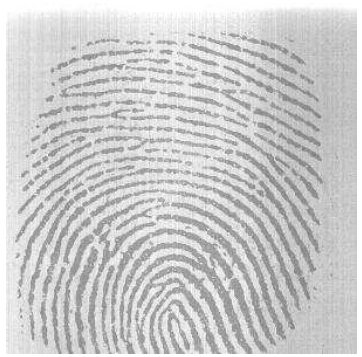

(b)

Figure 2.9: Sample fingerprints from Digital Biometrics (DBI) and VERIDICOM (VERI) sensors. (a) Image acquired from DBI. (b) Image acquired from VERI.

obtained using sensors of similar resolution, have very different spatial characteristics.

The fingerprint data of 128 different non-habituated cooperative subjects was made available ${ }^{5}$. All subjects provided 4 impressions each of 4 distinct fingers using both the sensors. Thus, 2,048 fingerprint impressions (4 impressions each of 512 different fingers) were available for each sensor. Two different minutiae-based matchers - the BOZORTH3 matcher developed by NIST ${ }^{5}$ and the VeriFinger matcher developed by Neurotechnologija ${ }^{6}$ - were used in our experiments (details regarding matchers are provided in the Appendix $(\mathrm{B})$.

Pre-calibration minutiae matching results using the VeriFinger matcher are shown in Fig. 2.10. The matching minutiae points, as estimated by this matcher, are highlighted in this figure. Fig. 2.11 illustrates the difference in the minutiae count between the partial prints from VERI and the (almost) full prints from DBI. It can be observed that the DBI images contain more minutiae compared to the VERI images. This can be further confirmed in Fig. 2.10(c) and 2.10(f) where the number of overlapping minutiae is observed to be relatively more in the DBI image pair. Further, note that during inter-sensor matching (Fig. 2.10(i)), the VeriFinger matcher is unsuccessful in correctly detecting corresponding minutiae pairs.

The calibration model described in the previous section was applied at 2 different levels Feature level (minutiae) and Image level. The calibration model itself was computed using 8 different representative image pairs obtained from the two sensors. In our experiments, the

\footnotetext{
${ }^{5}$ Although data from more number of users was collected, only 128 of these users had data pertaining to both the sensors. For the remaining users, corresponding images could not be determined.

${ }^{5}$ http://fingerprint.nist.gov/NFIS/

${ }^{6}$ http://www.neurotechnologija.com/
} 


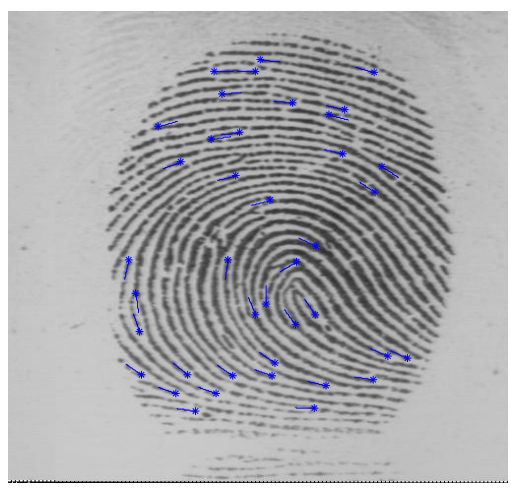

(a) DBI Fingerprint 1

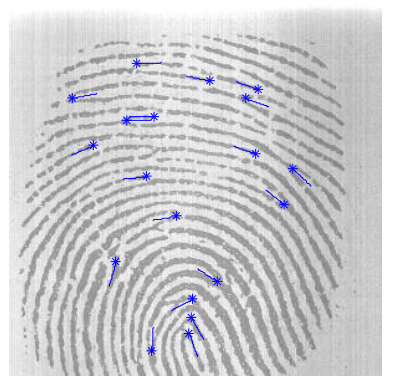

(d) VERI Fingerprint 1

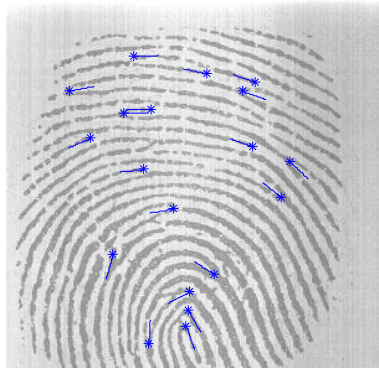

(g) VERI Fingerprint 1

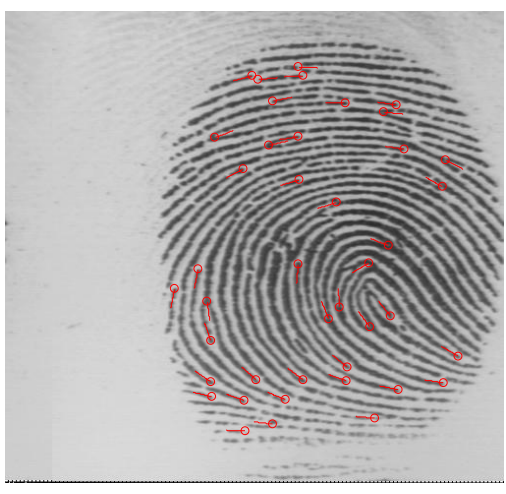

(b) DBI Fingerprint 2

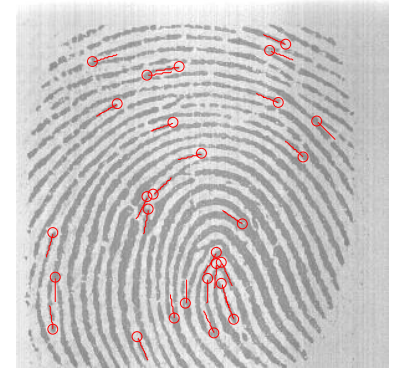

(e) VERI Fingerprint 2

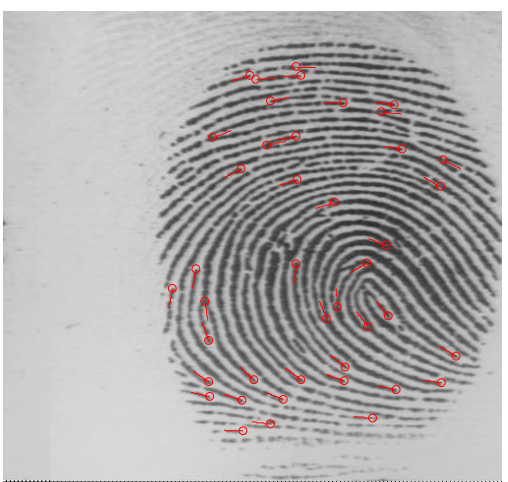

(h) DBI Fingerprint 2

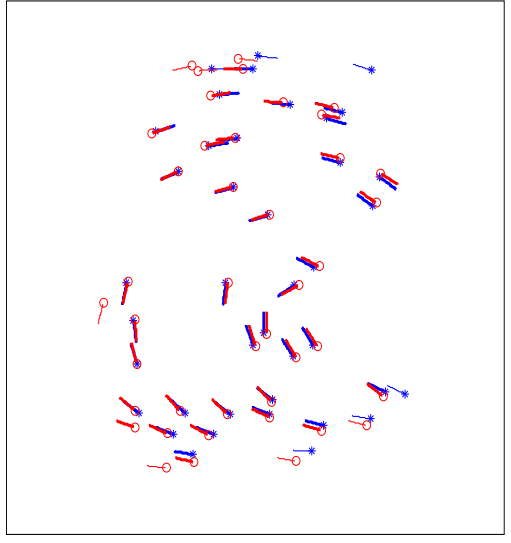

(c) DBI vs DBI Matching

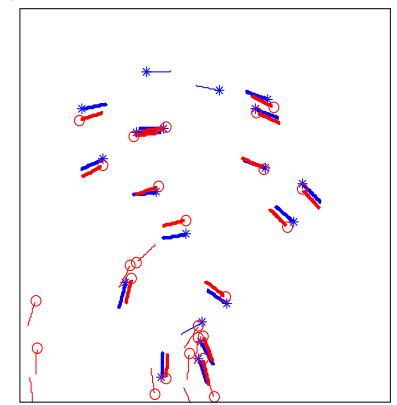

(f) VERI vs VERI Matching

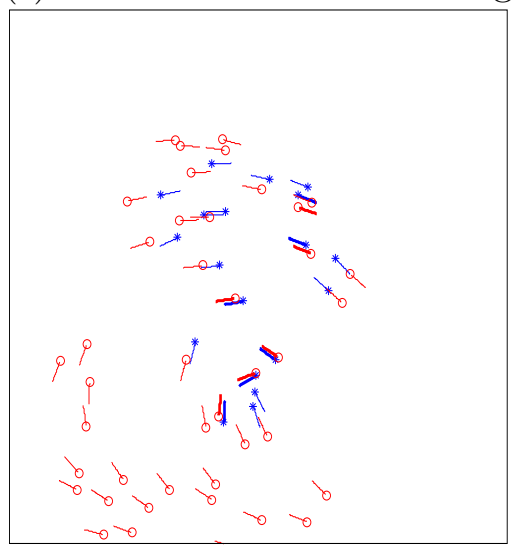

(i) VERI vs DBI Matching

Figure 2.10: Fingerprints in the first 2 columns are matched and the corresponding minutiae matching results are shown in the third column. Highlighted minutiae represent the minutiae that have been matched. The first row illustrates DBI vs DBI matching, the second VERI vs VERI matching and the third VERI vs DBI matching. 


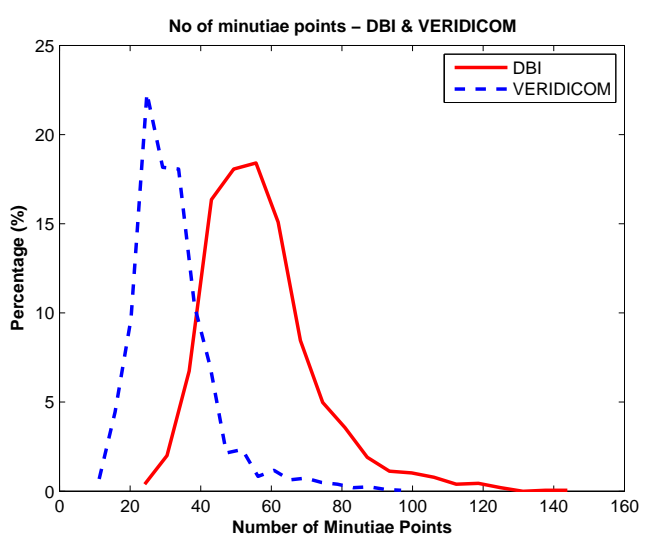

Figure 2.11: The histogram of minutiae points extracted from the DBI and VERI images.

VERI sensor was calibrated with respect to the DBI sensor. An illustration of the calibration process using mesh-grid plots can be seen in Fig. 2.12. Here, each mesh-grid represents the spatial geometry of individual images.

Five different matching experiments were conducted using each of the two matchers in order to demonstrate the benefit of the calibration model:

(a) DBI vs DBI : The genuine and impostor match scores were generated by comparing minutiae sets within the DBI database.

(b) VERI vs VERI : The genuine and impostor match scores were generated by comparing minutiae sets within the VERI database.

(c) VERI vs DBI (before calibration): The genuine and impostor match scores were generated by comparing the VERI minutiae sets with the DBI minutiae sets.

(d) VERI vs DBI (after minutiae calibration): The minutiae sets extracted from the VERI images were subjected to the average deformation computed using our calibration model before matching them against the minutiae sets of the DBI images. An illustration of the original minutiae set of a VERI image along with the calibrated minutiae is provided in Fig. 2.13(a and b). In the current formulation, the location and the orientations of individual minutia points are perturbed. The location is computed using the average deformation while the orientation is manipulated as shown in Section 2.4.3.

(e) VERI vs DBI (after image calibration): The calibration model suggested here was also used to calibrate the images acquired using the VERI scanner before extracting the minutiae from them. However, the application of a non-linear transformation to individual image pixels will generate sub-pixel information which can confound the minutiae detection process. In Fig. 


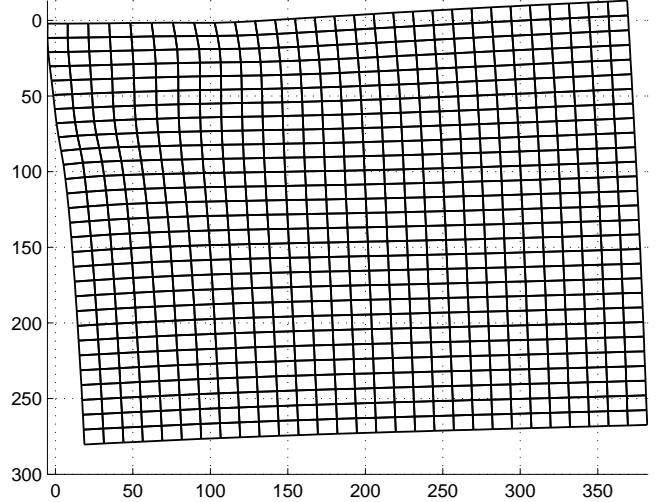

(a)

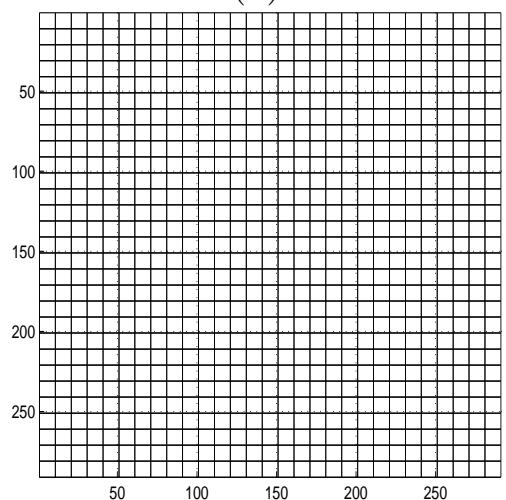

(c)

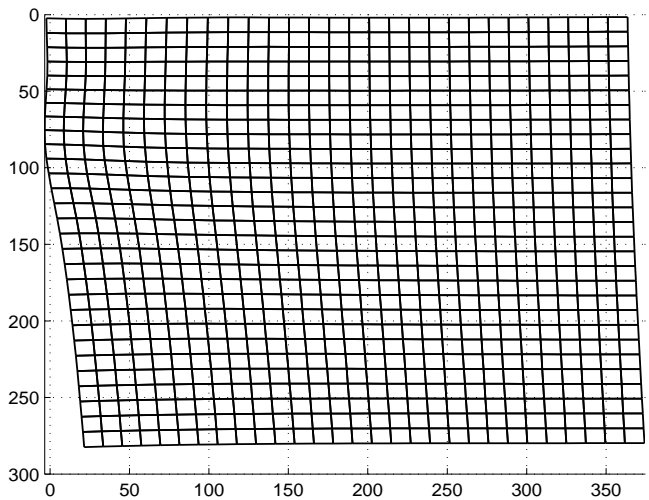

(b)

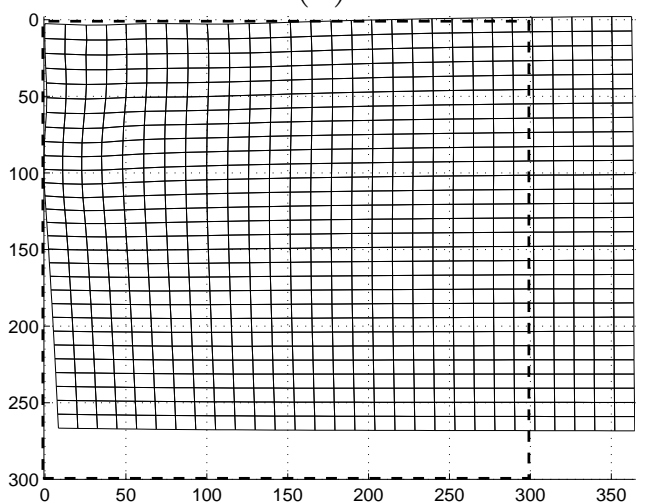

(d)

Figure 2.12: Demonstration of the calibration process using mesh-grid plots. (a) and (b) The deformation of two VERI images with respect to their corresponding DBI images as estimated by the TPS model. (c) A pre-calibrated VERI image. (d) The post-calibrated VERI image based on the deformation of eight representative image pairs. The dashed rectangle in (d) indicates the dimensions of the original VERI image. 
2.14, the original fingerprint image along with the image generated after applying the non-linear transformation is shown. As seen in 2.14(b), some pixel information is lost due to the non-linear transformation. In some cases there could be a substantial perturbation of ridge information, leading to erroneous minutiae detection. One way to address this issue would be to subject the image to a simple affine transformation (predominantly scaling along the $\mathrm{X}$ - and $\mathrm{Y}$ - directions) before extracting minutiae points. These scaling factors were calculated as the average of the corresponding affine parameters computed by the calibration model based on the 8 representative image pairs (refer to Section 2.4.4). The mesh grid plot along with the minutiae obtained after image calibration are shown in Fig. 2.13(c). As the minutiae are extracted from the images obtained after calibration, the minutiae count might be altered.

A total of 3,072 genuine $\left(512 *\left(\begin{array}{l}4 \\ 2\end{array}\right)\right)$ genuine scores and $523,264\left(\left(\begin{array}{c}512 \\ 1\end{array}\right) *\left(\begin{array}{c}511 \\ 1\end{array}\right) *\left(\begin{array}{l}4 \\ 1\end{array}\right) / 2\right)$ imposter scores were generated for each of the five matching scenarios.

The Receiver Operating Characteristic (ROC) curves summarizing the performance of these five experiments using both the matchers is shown in Fig. 2.15. It is observed that the proposed calibration model results in improved inter-sensor matching performance. For example, in the scenario involving VERI vs DBI (after minutiae calibration) the GAR (Genuine Accept Rate) increases from $\sim 35 \%$ to $\sim 75 \%$ at a FAR (False Accept Rate) of $0.01 \%$ when the VeriFinger matcher is used. Similarly, when the BOZORTH3 matcher is used, the GAR increases from $\sim 35 \%$ to $\sim 70 \%$ at the same FAR. Similar observations can be made in the case of image calibration. Fig. 2.16 indicates the improved minutiae matching as a result of calibration.

Thus, we observe that a simple non-linear calibration scheme is sufficient to address the problem of interoperability. This, however, does not undermine the development of sophisticated matching algorithms that utilize information such as ridge counts between minutiae points when comparing minutiae sets pertaining to multiple sensors. Such approaches have their own merits since they would preclude the establishment of manual correspondences.

\subsubsection{Role of scaling parameters}

In the sensor pair considered in our experiments, the scaling parameters along the horizontal $(\mathrm{U})$ and vertical $(\mathrm{V})$ directions are observed to be more prominent than the local deformations. In Table 2.2, the scaling factors $S_{u}, S_{v}$ for all the 8 representative images are shown. Fig. 2.17 shows the contribution of the non-linear parameter weights, where it can be seen that most of 


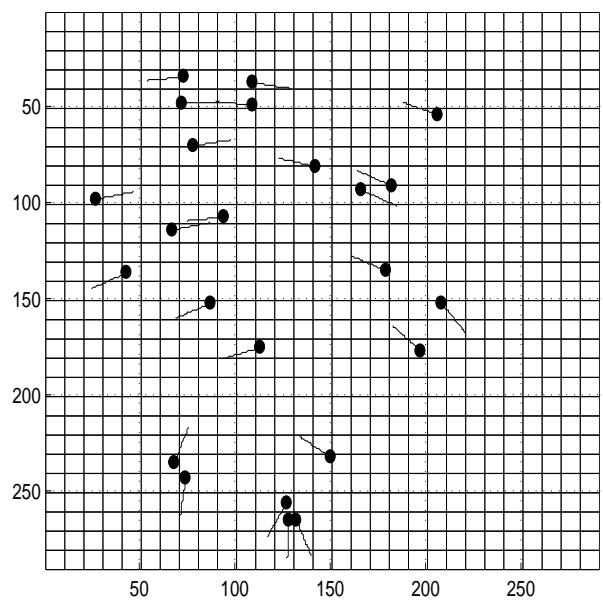

(a)

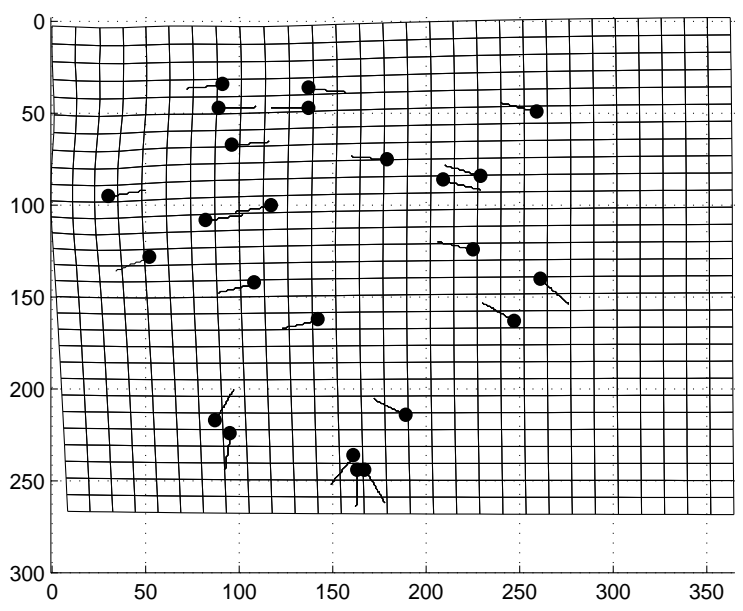

(b)

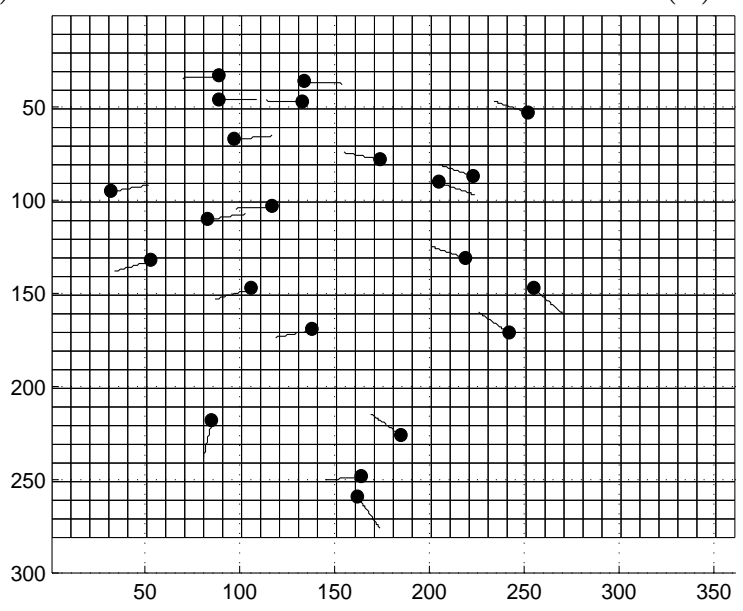

(c)

Figure 2.13: (a) Original mesh-grid plot with minutiae (b) Mesh-grid plot with calibrated minutiae (after minutiae calibration) (c) Mesh-grid plot with minutiae (after image calibration)

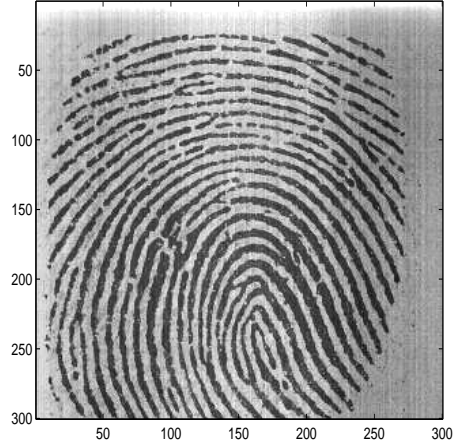

(a)

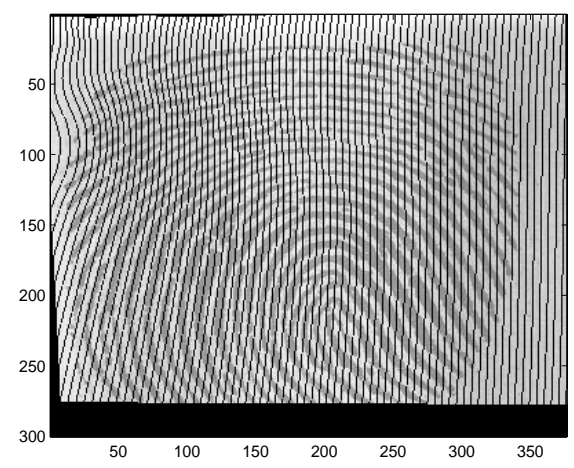

(b)

Figure 2.14: (a) Original fingerprint image. (b) Image generated after applying non-linear transformation leads to loss of information.

Table 2.2: TPS scaling parameters for the representative images

\begin{tabular}{|l|l|l|l|l|l|l|l|l|l|}
\hline$S_{u}$ & 1.2315 & 1.2638 & 1.1851 & 1.2146 & 1.2263 & 1.2054 & 1.1272 & 1.2011 & $S_{U}=1.2069$ \\
\hline$S_{v}$ & 0.9949 & 0.9040 & 0.9907 & 0.9801 & 0.8965 & 1.0090 & 0.8245 & 0.8738 & $S_{V}=0.9342$ \\
\hline
\end{tabular}




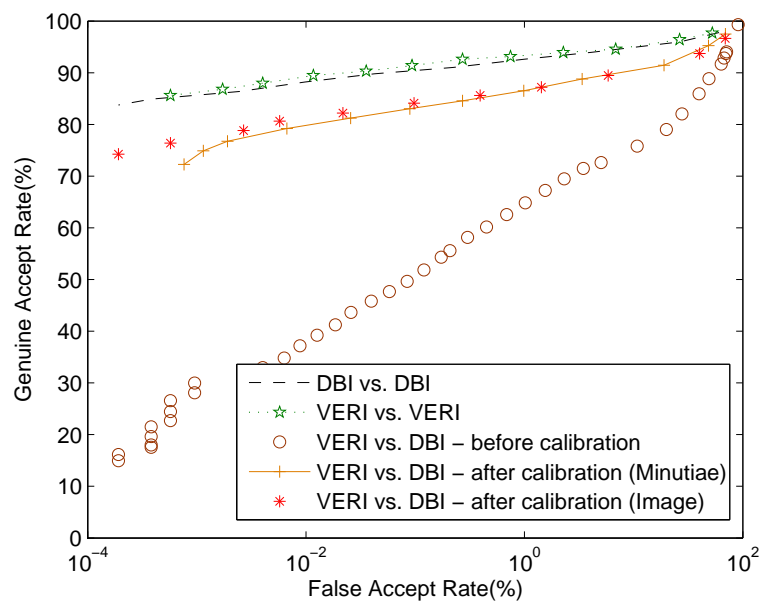

(a)

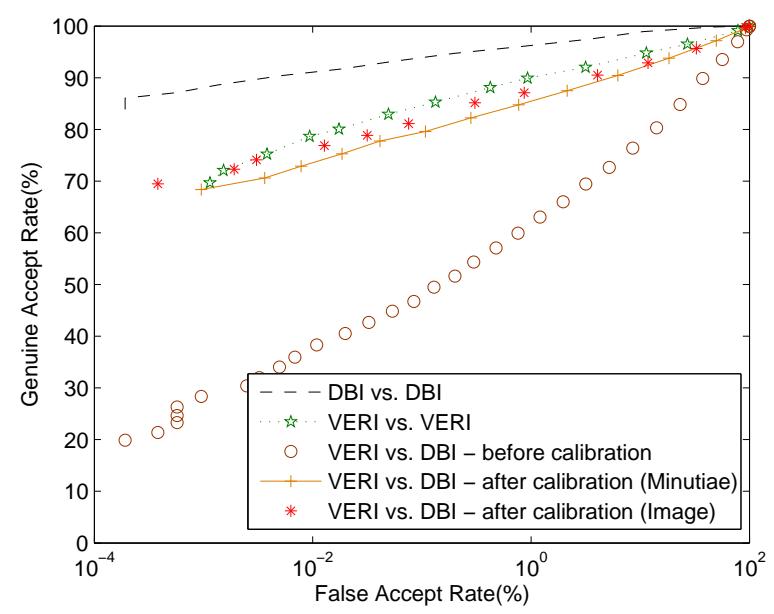

(b)

Figure 2.15: ROC curves indicating the improvement in inter-sensor performance on the MSU database. (a) VeriFinger matcher. (b) BOZORTH3 matcher.

the weights are close to the zero value and, hence, are less significant. Thus, from Table 2.2 and Fig. 2.17 it can be concluded that for this specific database, the scaling factors are more significant in comparison with the non-linear weights.

The prominence of scaling factors are observed for the current database, but for some other databases the non-linear weights might be more significant. In such a scenario, the calibration based on scaling factors approach will not work. Also in cases of non-linear distortion (like trapezoidal distortion), the non-linear weights will not be close to zero.

For the current database, as the scaling factors are prominent over the non-linear parameters, analysis of the effect on performance using the scaling factors for minutiae was conducted. For the sake of this analysis, the location $(u, v)$ and orientation $(\theta)$ values of the minutiae sets derived before calibration were manipulated on basis of the scaling factors derived in Eq. 2.6. The new $\left(u^{\prime \prime}, v^{\prime \prime}\right)$ and orientation $\left(\theta^{\prime \prime}\right)$ values are computed as shown in Eq. 2.7.

$$
u^{\prime \prime}=u S_{U} ; \quad v^{\prime \prime}=v S_{H} ; \quad \theta^{\prime \prime}=\tan ^{-1}\left\{\frac{S_{V}}{S_{U}} \tan (\theta)\right\} .
$$

The performance after modifying the original minutiae template from $(u, v, \theta)$ to $\left(u^{\prime \prime}, v^{\prime \prime}, \theta^{\prime \prime}\right)$ can be observed in Fig. 2.18 (VERI vs DBI - after calibration (minutiae scaling)). This VERI vs DBI - after calibration (minutiae scaling) performance is almost comparable with VERI vs DBI - after calibration (image as well as minutiae), for both the matchers. This validates our observation of prominence of linear scaling factors across images, procured from both the sensors. One must keep in mind that the linear calibration approach would only work in presence of 


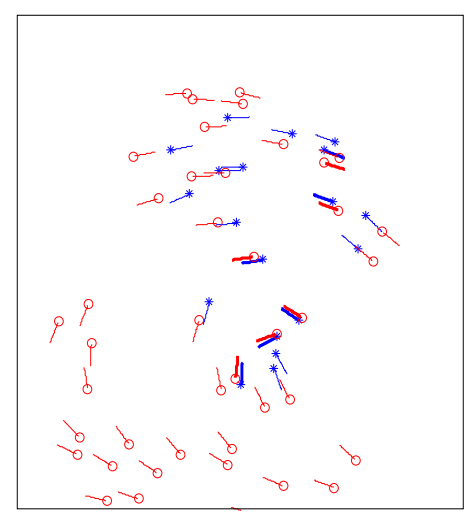

(a) VERI vs DBI before calibration

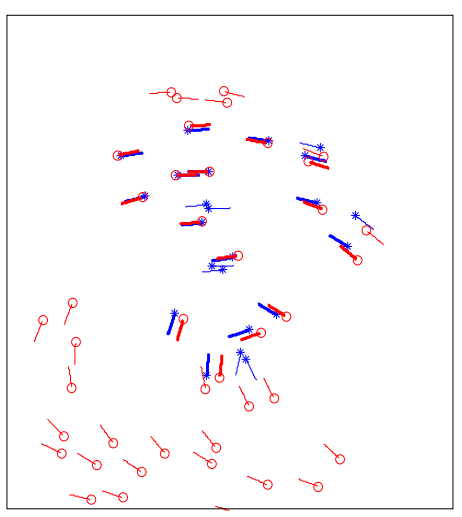

(b) VERI vs DBI after calibration (Image)

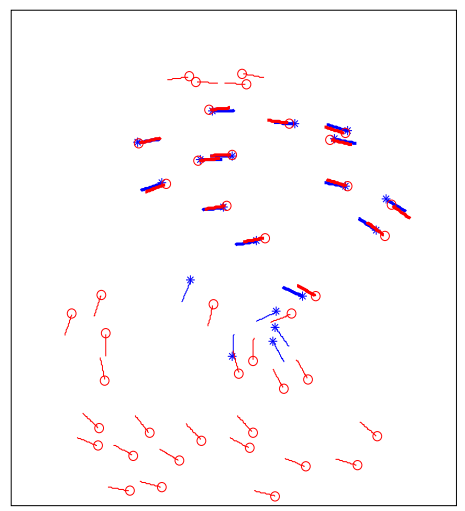

(c) VERI vs DBI -

after calibration (Minutiae)

Figure 2.16: Improved inter-sensor minutiae matching after calibration. (a) indicates the matching minutiae before calibration, (b) and (c) show the matching minutiae after image and minutiae calibration, respectively. Matching minutiae pairs are highlighted for distinguishing them from the non-matching minutiae pairs. Match scores as assessed by the VeriFinger matcher for (a), (b), (c) are 35, 193 and 202, respectively. 


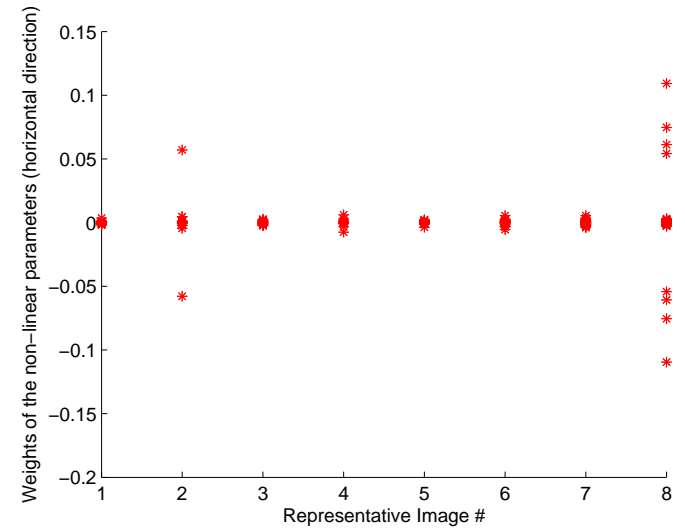

(a)

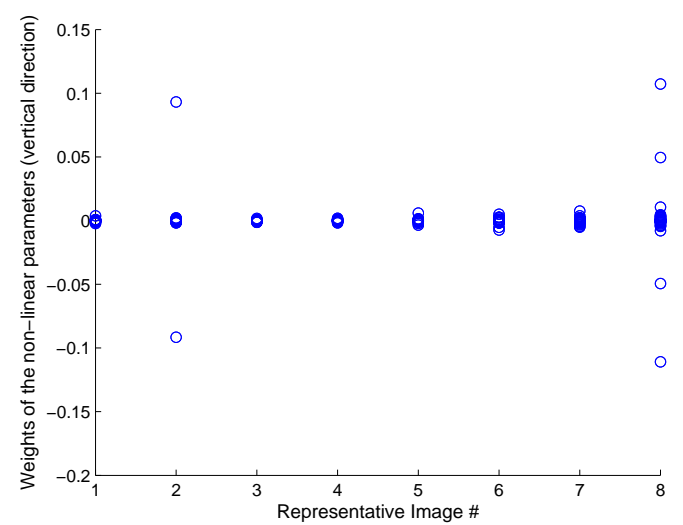

(b)

Figure 2.17: Plot indicating the distribution of weights for the non-linear parameters (derived using TPS) for each of the 8 representative images. (a) Weights computed in the horizontal direction. (b) Weights computed in the vertical direction. The $\mathrm{x}$-axis corresponds to the representative image pair while the y-axis represents the weights of the non-linear parameters.

prominent linear scaling factors. In cases of nonlinear distortion, e.g., trapezoidal distortion, this particular approach of linear calibration would not suffice. The comparable performance of minutiae and Image calibration as well as minutiae scaling, indicates the potential of this scheme to work accurately even in the absence of scaling factors and prominence of non-linear weight parameters.

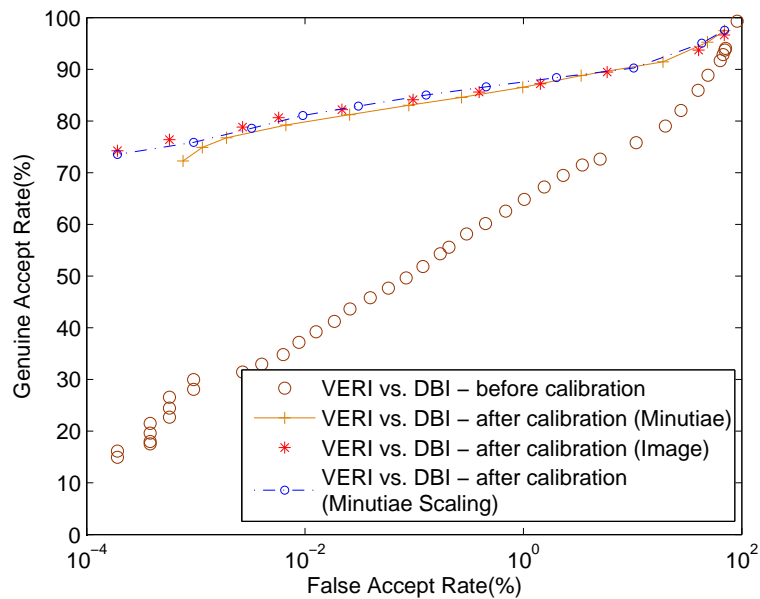

(a)

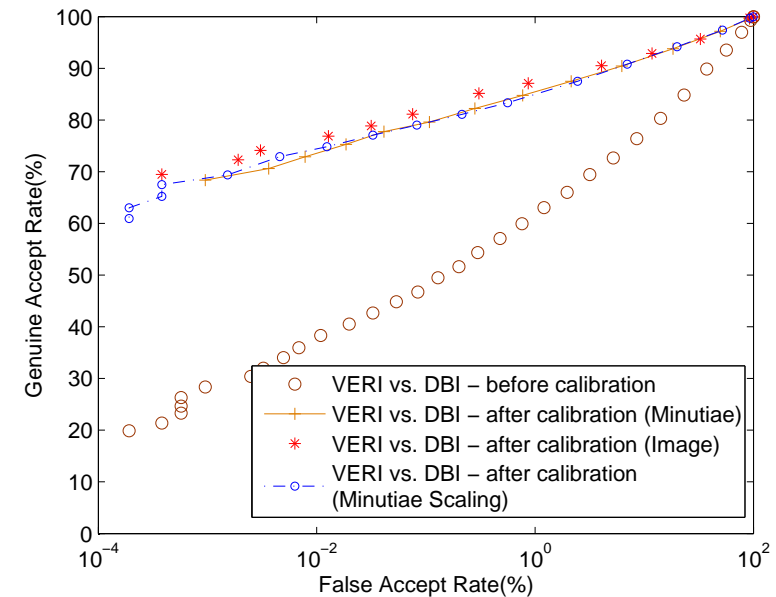

(b)

Figure 2.18: ROC curves comparing the minutiae scaling performance with non-linear image and minutiae calibration. (a) VeriFinger matcher. (b) BOZORTH3 matcher. 


\subsection{Summary and Future Work}

In this work we have demonstrated that a simple non-linear calibration scheme based on the thin-plate spline model is sufficient to handle variations in minutiae distributions across multiple sensors. The parameters of the model (average deformation) are computed based on a small representative set of image pairs containing control points (landmarks) whose correspondences are manually established. The average deformation is used to distort the minutiae points of images acquired using one sensor before comparing them with the minutiae points of images corresponding to another sensor. A significant performance improvement is observed when the proposed scheme is utilized to compare fingerprint images originating from two different sensors, viz., optical and solid state capacitive sensors. Only a few representative image pairs are needed for the successful implementation of the proposed method. In future, we plan to use more sophisticated calibration grids (similar to camera calibration in computer vision) by imaging rigid finger-like synthetic material with pre-established control points. This would avoid issues related to the user-dependent elasticity of the skin.

An inherent limitation of our proposed scheme could be the inability to compensate for variations introduced in fingerprint images acquired across sensors with significant differences in resolution (e.g. 250 dpi fingerprint images matched against 1000 dpi fingerprint images). The 1000 dpi images reveal level III fingerprint features such as pores on the surface of the skin. It is not possible for an image captured using a $250 \mathrm{dpi}$ sensor to reveal these intricate details (i.e, pores). Thus, a simple non-linear transformation will not register such disparate images.

A generic calibration model, based on automated control point selection from the representative image pairs, is essential to facilitate interoperability across a wide range of sensors. In the formulation presented in this work, variations introduced in the images and their corresponding features are compensated by defining the solution as a simple transformation function between the extracted features. This approach needs to be evaluated across images acquired using different sensors. To study the feasibility of the proposed approach across multiple sensors, currently we are acquiring data from 3 different sensors (Crossmatch Verifier 300, Secugen Hamster III, Precise $100 \mathrm{AX}$ ) in order to study the interoperability issues associated with them. We are also developing alternate fingerprint matching schemes to address interoperability between minutiae sets obtained from multiple sensors. 


\section{Chapter 3}

\section{Fingerprint Image Quality}

\subsection{Introduction}

Fingerprint quality is usually defined as a measure of the clarity of the ridge and valley structures, as well as the "extractability" of features (such as minutiae and singularity points) [10]. Minutiae are usually extracted after segmenting the image into foreground and background. Foreground is the fingerprint area within the captured image where a prominent ridge-valley pattern is observed while the area not containing any ridge-valley pattern is classified as Background. Fig. 3.1(a) shows the original image and fig. 3.1(b) shows the foreground area segmented from the background area.

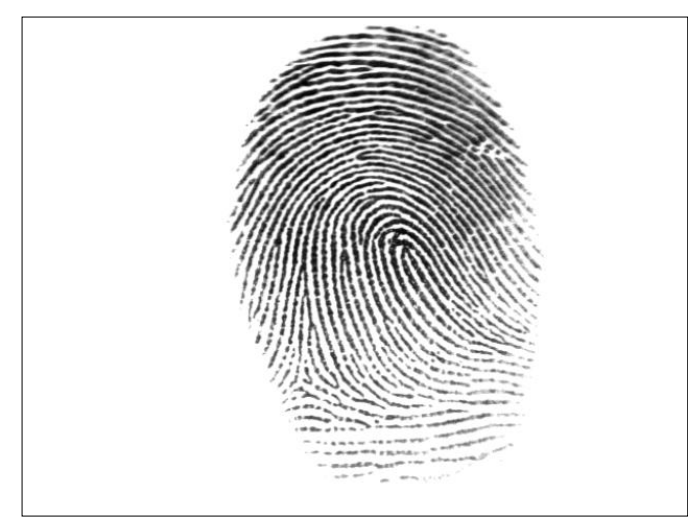

(a)

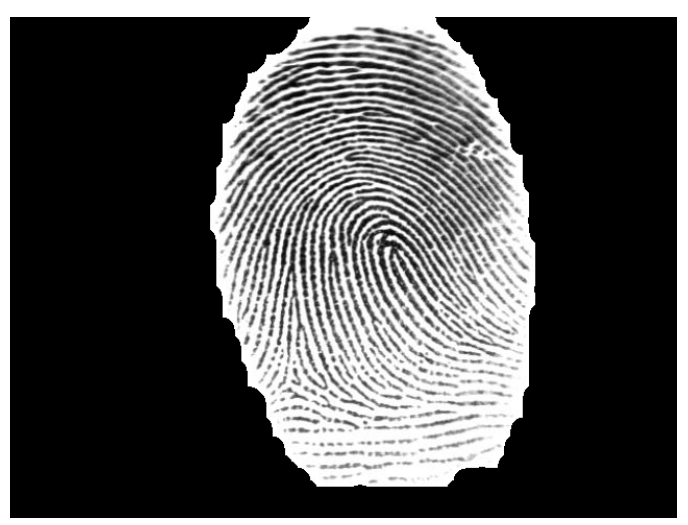

(b)

Figure 3.1: (a) Original Fingerprint, (b) Segmented Fingerprint

Within the foreground, if the captured ridge-valley pattern is prominent and clear then the fingerprint is said to be of good quality, whereas if the captured ridge-valley pattern is not clear 
(contains some noise or irregularities) then the fingerprint is said to be of bad/poor quality. Many different approaches have already been developed for estimating quality of a given fingerprint image, a review of which is presented in the next section.

The remainder of the chapter is organized as follows. In Section 3.2 some of the already existing quality measures are reviewed; Section 3.3 discusses a gabor-based classification approach for classifying fingerprint images into good, dry and smudged quality. The results and the limitations of the proposed scheme are covered in the same section; Section 3.4 presents a novel approach of incorporating local feature quality into the score formulation. This section also includes the experimental results on multiple databases for different matching schemes.

\subsection{Quality Estimation Techniques}

Different approaches of quality estimation are summarized in this section. The individual approaches are grouped into 5 different categories: (a) Intensity-based, (b) Filter-based, (c) Classifier-based, (d) Wavelet-based and (e) Hybrid approaches.

\subsubsection{Intensity-based Quality Estimation}

Bolle et. al [43], 44] use the ratio of directional area to the non-directional area towards computing quality. Directional area is defined as an area having dominant direction or a prominent ridge-valley pattern while a non-directional area does not have a dominant direction or a prominent ridge-valley pattern. A metric signifying the dryness and the smudginess in a fingerprint image has also been derived.

Variation in the intensities around a pixel are calculated along different directions. The intensity differences are computed between the pixel of interest and its corresponding neighboring pixels along the respective direction. The minimal intensity variation along an orientation gives the orientation of the pixel. If the sum of the variation in intensities along all the directions is less than a background threshold (predetermined) for each direction, the pixel is classified as a background pixel. When more than a certain fraction of all pixels within a block are background pixels, the block is classified as a background block. Using connected component analysis, if the area of a component within a block is greater than a certain fraction of the block image area, the block is defined as a foreground block. After determining the foreground blocks, the dominant 
direction within each block is calculated. A histogram of orientations for each pixel within a block is computed and if the histogram has a maximum value greater than a certain threshold, then the block is labeled as prominent. Inconsistencies within "directional" and "non-directional" blocks are removed by looking at the neighboring blocks. Finally, using connected component analysis, regions of dominant directional blocks within an area smaller than a threshold number of blocks are discarded. This results in foreground image which is partitioned into region of blocks with direction and blocks without direction. Weights are assigned to foreground blocks with respect to the centroid location of foreground. Quality of the image is calculated as the ratio of total weights of blocks having dominant direction to total weights of foreground blocks. The objective here is to detect blocks with dominant direction exhibiting a clear ridge/valley pattern.

The dryness and smudginess of a poor fingerprint image is also evaluated. Mean intensity of all pixels within a foreground block is calculated. Pixels having a value less than the mean intensity are considered as pixels on a ridge. Mean intensity of ridge pixels is calculated and the standard deviation of all pixels within the same block is also calculated. For smudged blocks (low contrast), the mean and standard deviation is small. The block is classified as a smudged block if the product of the mean and standard deviation is smaller than a threshold. Smudginess measure is determined by the fraction of smudged blocks within the foreground area. Dry blocks (low contrast) have a large mean and a small standard deviation. The block is classified as a dry block if the ratio of the mean and standard deviation is greater than a certain threshold. Dryness measure is computed similar to the smudginess measure.

Park et. al [45] have proposed a similar approach in which a $16^{*} 16$ block size is considered. The average intensities in 8 different directions within each block are recorded and the difference between the maximum and the minimum value defines the quality of the block (QB). A threshold distinguishes a good quality block from a poor quality block. The percentage of good quality and poor quality blocks within the foreground is computed and another threshold determines the nature of the print, namely good or poor quality.

An approach on parallel lines has been proposed by Jain et. al [46] where in they calculate the variance across ridge direction. The quality of an image is defined based on the calculated variance and a goodness index is also defined which compares the minutiae extraction procedure with the actual minutiae extracted by an human expert. The quality of a fingerprint is estimated through the variance calculated perpendicular to the orientation field. For the foreground, vari- 
ance along the ridge direction would be low whereas variance across the ridges would be fairly high. The background ideally has low variance in all the directions. This variance is used to quantify the quality of each block. According to the value of the variance a "Good", "Medium", "Poor" and "Background" quality is assigned to each block.

\subsubsection{Filter-based Quality Estimation}

An approach to determine fingerprint image quality on the basis of Gabor features is presented by Shen et. al [47]. The image is divided into blocks and Gabor features for each block are calculated. The quality is determined by the standard deviation of these Gabor features. For a good quality image block with local ridge orientation, the values of one or more Gabor features are larger than the rest of the features. For a poor quality image block without local ridge orientation, the values of the Gabor features are closer to each other. Thus, the standard deviation can be used for quality estimation as well as foreground or background segmentation. A pre-defined threshold is used for labeling the block as foreground or background. The foreground block is classified into "poor" or "good" on the basis of another threshold. The quality index is computed as a fraction of good quality blocks within the foreground area. If this quality index is greater than a threshold, the image is classified as "good" quality else the image is classified as a "poor" quality image.

The smudginess index and dryness index are also determined for a "poor" quality image, based on the mean pixel value within the block. For a smudged block, the mean value for the block would be small while for a dry block it would be large. Thresholds are set to classify the blocks as smudged or dry and also to classify the image as smudged or dry (similar to [43]).

Pardo et. al [48] have used a similar Gabor feature-based approach. A global quality measure is defined which takes into account the whole fingerprint while a local quality measure is defined which considers the region surrounding singularities. The local features are the extracted singularities, core and delta points. For accurate singularity detection, anisotropic diffusion is used as a pre-processing step for smoothing the image. The number of iterations required by anisotropic diffusion is derived empirically for the specific image quality as this technique is built towards classification. 200 iterations are required to correctly classify poor quality fingerprints, less are required for good quality fingerprints and more for bad quality fingerprints.

For a block size of $16 \times 16,8$ different Gabor features are derived for each block (similar 
to [47]). If the standard deviation of Gabor features of the block is less than a threshold then the block is marked as a good quality block else it is marked as a bad quality block. The global quality is defined similar to the one in [47]. The local quality is defined in an area surrounding the singularity points called the mask and is given by the proportion of the bad quality blocks in the mask w.r.t. the total number of blocks in the mask. The fingerprint is eventually accepted or rejected on basis of local quality, global quality and the number of iterations used for anisotropic diffusion.

\subsubsection{Classifier-based scheme}

NIST $^{11}$ has developed a measure to assess the fingerprint image quality [4]. Their algorithm associates a quality with each minutiae and also generates an quality map of the fingerprint image. The image is divided into a grid of blocks and several parameters corresponding to each block are calculated. These parameters aid in defining the low contrast, low flow, high curve and direction maps. Areas exhibiting low contrast, low flow and high curves are deemed to be unstable areas, where minutiae detection is considered to be unreliable. A reliability measure for the minutiae is extracted on basis of their location in the quality map.

Direction Map - The purpose of this map is to define areas with sufficient ridge-valley structure as minutiae can be reliably detected from these areas.

Low Contrast Map - The blocks with low contrast are flagged on basis of the pixel intensity distributions. If the distributions are too narrow, the blocks are classified as low contrast blocks, which include the background, smudged and dry blocks.

Low Flow Map - Low flow map marks the blocks that could not initially be assigned a dominant ridge flow. Minutiae detected from these areas are not reliable. Lower quality is assigned to the minutiae detected from these areas.

High Curve Map - Minutiae detected in the high curvature areas are not reliable, especially in the core and delta regions. Two measures, Vorticity and Curvature are used. Vorticity measures the cumulative change in ridge flow direction around all neighbors of the block while curvature measures the largest change in direction between a block's ridge flow and the ridge flow of each of its neighbors. Lower quality is assigned to the minutiae detected from these areas as well. Quality Map - This is the final image map obtained from the low contrast, low flow, high curve

\footnotetext{
${ }^{1}$ National Institute of Standards and Technology
} 
and direction maps. Five levels of quality are assigned with 0 being the background and 4 being a very good region. The quality assigned to a specific block is determined based on its proximity to the flagged blocks in the above mentioned maps.

Assess Minutiae Quality - MINDTCT [49] is the minutiae extraction algorithm used by NIST. Poor quality or spurious minutiae are detected by associating a reliability measure on the basis of two factors: (a) the quality $(L)$ of a minutiae point based on its location in the quality map and (b) mean and standard deviation of the pixels within an immediate neighborhood of the minutiae location (neighborhood size 11 pixels). For a high quality region, the mean pixel intensity would be very close to 127 while the standard deviation would be greater than or equal to 64 . Reliability measure is calculated based on the mean and standard deviation of the minutiae neighborhood. Minutia quality is calculated using the quality map and the reliability measure.

Feature vectors are defined on basis of the quality map and the assessed minutiae quality. The feature vector $\left(v_{i}\right)$ is constructed by considering 11 different features derived from: the number of foreground blocks, percentage of foreground blocks belonging to certain quality (associated with the quality map) and count of minutiae with differing quality values.

The purpose of this research is to define a fingerprint image quality which would predict the matcher's performance. Hence, good quality fingerprints are expected to fabricate high match scores and these scores should be well separated from the non-match distributions. Similarly, poor quality fingerprints are expected to generate lower match scores, lying in the region of overlap with non-match scores. Thus, a quality measure should be indicative of the degree to which the match distribution $M\left(s_{m}\right)$ is separated from the non-match distribution $N\left(s_{n}\right)$, where $s_{m}$ and $s_{n}$ are the matched and non-matched scores respectively. Now, if the Gallery $\Gamma$ represents the set of enrollees in a biometric system while Probe set $\Pi$ serves as a set of legitimate users of the system, then the estimated quality measure of biometric sample $x_{i}$ is defined as

$$
o\left(x_{i}\right)=\frac{s_{m}\left(x_{i}\right)-E\left[s_{n}\left(x_{j i}\right)\right]}{\sigma\left(s_{n}\left(x_{j i}\right)\right)} \forall x_{i} \varepsilon \Gamma \text { or } \Pi
$$

where, $o\left(x_{i}\right)$ is the normalized match score of sample $x_{i}, E[$.$] is the expected value, \sigma($.$) is the$ standard deviation, $s_{m}\left(x_{i}\right)$ is the match score of $x_{i i}$ (also represented as $\left.x_{i}\right)$ and $s_{n}\left(x_{j i}\right)$ are the non-match scores of the sample $x_{j i}$. For each fingerprint, image quality is defined as the predictor of its normalized match score. To obtain the estimated quality measure $q_{i}$ for the biometric sample $x_{i}$, a feature vector $v_{i}$ consisting of all appropriate signal and image fidelity 
characteristics is generated and then $v_{i}$ is non-linearly mapped to obtain $o\left(x_{i}\right)$.

$$
\begin{aligned}
v_{i} & =L\left(x_{i}\right) \\
q_{i}=\tilde{o}\left(x_{i}\right) & =I\left(v_{i}\right)
\end{aligned}
$$

where, L(.) gives the feature vector described above (11 different features extracted by MINDTCT) and $\mathrm{I}($.$) is the mapping from the space of feature vectors v$ to normalized match scores o(.). $\tilde{o}\left(x_{i}\right)$ is the predicted value of $o\left(x_{i}\right)$.

The 11-dimensional feature vector is provided as an input to the neural network which then acts as a classifier to define five different quality classes. The neural network is first trained and then tested on various databases. The final five classes representing the quality of the fingerprint image (outputs from the neural network) are "poor", "fair", "good", "very good" and "excellent".

\subsubsection{Wavelet-based Quality Estimation}

A research report presented by Bolle and Ratha [50] estimates the image quality of a wavelet compressed fingerprint image. The quality is based on the cumulative energy present in the sub-bands of a wavelet compressed image. This procedure is only beneficial if a wavelet scalar quantization (WSQ) compressed image is available. The transform attempts to concentrate the signal energy in a small number of transform coefficients. This method could be advantageous as wavelets provide a compact representation scheme and the image quality can be decided from the partially decompressed image. One can use this decision, to determine if one can proceed with the processing of this image. Also, one does not have to decompress the image to determine the image quality.

The energy in each of the sub-bands is computed from the wavelet coefficients. It is observed that the normalized cumulative energy in the initial few sub-bands of a good quality fingerprint is much higher than that in a poor quality fingerprint. The rate of growth of the cumulative energy is significantly different for the good and poor quality fingerprints. For poor quality images, the energy distribution in the low order sub-bands is more or less the same, while for a good quality image the energy is concentrated in a few selected sub-bands. 


\subsubsection{Hybrid Approaches}

\section{Intensity and Orientation-based Quality Estimation}

Lim et. al. [51] have proposed a quality and validity analysis technique based on both the local and global structures of the fingerprint image. First, the quality of the image is determined and then, invalid images are distinguished from the valid images. For the local structure, the local orientation certainty and the ridge-valley structure is considered. For global justification, the continuity of ridge orientation and the variation in the ridge to valley ratio is analyzed.

During local analysis, at every pixel within a block, the gray level gradients along the $\mathrm{x}$ and y-direction are calculated representative of the orientation. The covariance matrix of the gradient vector for all the pixels within a block, provide the eigen values for the block. For each block the ratio between the eigen values, defines the orientation certainty level which gives an indication of the strength of energy concentration along the ridge-valley orientation. The lower the value, the greater the energy. As some of the residue images have a strong orientation strength, ridge-valley structure is also examined. A grey level plot of an image block in the direction normal to the ridge flow is plotted. Three successive local maxima and minima are recorded and their average provides a threshold value for the separation of ridges and valleys. Ridge frequency value, ridge-to-valley thickness ratio and ridge thickness are also calculated from the grey level plot described above. An adaptive thresholding technique is implemented which identifies a block as a "blank" or "non-blank" identity. The non-blank blocks are then classified as "good", "bad" and "undetermined" on basis of orientation certainty and ridge-valley structure. The total quality score for local analysis is computed as a function of the total number of good, undetermined and bad quality image blocks respectively.

In global analysis, orientation along each row and column of the image blocks is observed for the detection of smooth or abrupt changes in the fingerprint image. The blocks which show abrupt changes in orientation are added to obtain a global orientation score. The ridge-to-valley thickness ratio calculated in the earlier step is used for determining the global quality as well. The standard deviation of this ratio from the mean value is used to quantify the image. Large deviation from the mean ratio value is used as a parameter to identify a bad quality from a good quality image. The total image quality is calculated as a function of the local quality measure, global orientation score, ridge-to-valley thickness, number of blank blocks obtained after adaptive 
thresholding and a heuristic representing the minimum number of foreground blocks required for a fingerprint image to be identified as one with sufficient information.

Hong et. al [8] derive several local features within a block on basis of the estimated orientation field. The ridges and valleys are modeled as a sinusoidal-shaped wave along the direction normal to the local ridge orientation. The amplitude, frequency and variance of the sinusoid are estimated, and used to classify the blocks as recoverable and unrecoverable. The image is then rejected, if the percentage of unrecoverable blocks exceeds a certain threshold.

\section{Filter and Intensity-based Quality Estimation}

Joun et. al [52 have defined three quality measures which aid in comparing the image qualities of infant and adult fingerprints.

Measure of Foreground portion - The variance in the gray-level pixel values is utilized for distinguishing the foreground from the background. Foreground intensities will have a relatively large variance compared to the background. Based on the magnitude of gray-level variance, each overlapping block is assigned to the foreground or the background. This scheme is similar to the one implemented by Jain et. al [46].

Measure of Directional Contrast - Local contrast in gray values between the ridges and the valleys along the local orientation of the ridge flow is used as a measure of directional contrast. For a good quality block, the ridges and the valleys are well separated and display a high directional contrast while a bad quality block would display a low directional contrast. A 8-directional filter is used in a moving window operation for computing the directional contrast.

Measure of Gabor Feature - The standard deviation of the Gabor features of blocks is used as a measure of image quality. This is similar to the approach put forth in Shen et. al [47].

These three quality measures are used as separate entities while analyzing the overall quality of the image. The average ridge distances between infant and adult fingerprints is also calculated. The lower quality of an infant fingerprint is a direct consequence of the average ridge distance being $84 \%$ of the adult average ridge distance. Hence, the average ridge distance can also be used as a quantifying measure. 


\section{Orientation and Frequency domain approach}

Chen et. al. [10] have defined a quality index in the spatial domain (similar to [51]). If $\lambda_{1}$ and $\lambda_{2}$ are the two eigenvalues, the coherence measure is computed as,

$$
\tilde{k}=\frac{\left(\lambda_{1}-\lambda_{2}\right)^{2}}{\left(\lambda_{1}+\lambda_{2}\right)^{2}}
$$

$\tilde{k}$ reflects the clarity of the local ridge-valley orientation in each foreground block. If a block has a distinct ridge-valley orientation, then $\lambda_{1}>>\lambda_{2}$ which results in $\tilde{k} \approx 1$. Else, if a block is of poor quality then $\lambda_{1} \approx \lambda_{2}$ which results in $\tilde{k} \approx 0$. A single quality measure $Q_{s}$ is defined as the weighted average of the block-wise coherence measures, where the weights are assigned on basis of the block location in the foreground area.

The global quality index is computed in frequency domain by considering the power spectrum of the fingerprint image. Good quality images display a prominent ring pattern in the power spectrum, corresponding to the consistent ridge-valley frequency. Poor quality images have a more diffused power spectrum due to non-uniform spacing between ridges along with the unclear ridges. Hence, the energy distribution in the power spectrum is utilized for computing a global quality index. Bandpass filters are designed to extract the energy content in each of the pre-defined ring-shaped concentric sectors. Energy concentration across these sectors aids in formulating the global quality index. For a good quality image, energy is concentrated in a few sectors while energy is more distributed in case of poor quality images.

\section{Filter, Feature and Foreground analysis-based approach}

In this approach proposed by Qi et. al. [53], seven different quality indices are combined into one single global quality measure. The first three measures are evaluated using Gabor filters, as shown in [47]. The first measure is calculated by averaging the standard deviation of gabor filters across all image sub-blocks. Second and third measures indicate the level of dryness and smudginess with the Smudginess and Dryness index (as computed in [47]). The next two features analyze the foreground area and the foreground position. The foreground area is a ratio of the number of foreground blocks with the total number of blocks. The foreground position is a measure of the deviation of the foreground centroid from the centroid of the sensor. Minutiae count index, the sixth measure, quantifies the similarity between the true extracted minutiae count with respect to expected minutiae count. The last measure is dependent on the presence 
or absence of the core singular point. Two different functions of combining these seven different quality factors are proposed in this paper.

A review of most of the quality measures is provided by Alonso-Fernandez et. al. [16]. The authors have grouped the techniques into local, global and classifier-based approaches. A comparison between the quality estimation techniques described in [10, [4] and [51] with the subjective image quality (as assigned by an human expert) is conducted.

\subsection{Gabor Analysis}

Most of the above mentioned techniques evaluate the fingerprint image quality and also associate a dryness or a smudginess measure with the image [43], [44], [47], [53]. Thus, dryness and smudginess are considered to be the most prominent factors towards poor quality prints. Hence, in the proposed quality analysis the primary aim is to segregate the good quality prints from the poor quality ones. The poor quality images are also classified into dry and smudged on basis of the nature of the prints.

\subsubsection{Structure of 2D Gabor Filter}

The general form of a 2D Gabor filter is given by

$$
\begin{aligned}
h\left(x, y, \theta_{k}, f_{t}, \sigma_{x}, \sigma_{y}\right) & =\exp \left[-\frac{1}{2}\left(\frac{x_{\theta_{k}}^{2}}{\sigma_{x}^{2}}+\frac{y_{\theta_{k}}^{2}}{\sigma_{y}^{2}}\right)\right] \times \exp \left(i 2 \pi f_{t} x_{\theta_{k}}\right) \\
k & =1 . . m \\
t & =1 . . n
\end{aligned}
$$

where $x_{\theta_{k}}=x \cos \theta_{k}+y \sin \theta_{k}$ and $y_{\theta_{k}}=-x \sin \theta_{k}+y \cos \theta_{k}, t$ denotes the number of frequencies, $f_{t}$ is the $t^{t h}$ frequency of the sinusoidal wave, $m$ denotes the number of orientations, $\theta_{k}$ is the $k^{\text {th }}$ orientation of the gabor filter and $\sigma_{x}$ and $\sigma_{y}$ are the standard deviations of the gaussian envelope along the $\mathrm{x}$ and $\mathrm{y}$ axis respectively. For a fingerprint image, $\mathrm{f}$ is the inverse of the average inter-ridge distance and $\theta_{k}=\pi(k-1) / m$, where $k=1 . . m$. Once these parameters are 
fixed, the magnitude of the Gabor feature at the sampling point $(i, j)$ is defined as,

$$
\begin{aligned}
g\left(i, j, \theta_{k}, f_{t}, \sigma_{x}, \sigma_{y}\right) & =\left|\sum_{x=-w / 2} \sum_{y=-w / 2}^{w / 2-1} I(i+x, j+y) h\left(x, y, \theta_{k}, f_{t}, \sigma_{x}, \sigma_{y}\right)\right| \\
k & =1 . . m \\
t & =1 . n
\end{aligned}
$$

where $I(a, b)$ denotes the gray-level value of the pixel $(\mathrm{a}, \mathrm{b})$ and $w$ is the size of the filter. Thus, $m \times n$ gabor features are obtained for each pixel. The whole image can be represented by $M \times N \times m \times n$ gabor features, where $M \times N$ is the image dimension.

\subsubsection{Advantages of Gabor Filter}

Fingerprints have a well defined local frequency and orientation [5]. In the proposed scheme, the underlying ridge-valley pattern of a fingerprint is analyzed using gabor filters. Properly tuned gabor filters can remove noise, preserve the true ridge and valley structures, and provide information contained in a particular orientation in the image [54], [55]. A minutia point can be viewed as an anomaly in locally parallel ridges and it is this information that can be captured using gabor filters [5], [56]. Gabor filters or filterbanks have been extensively used in the literature [5], [56], [57], [47], [48], as they are a well-known technique to capture useful information in specific bandpass channels as well as to decompose this information into biorthogonal components in terms of spatial frequencies [5], [56].

\subsubsection{Variations from the standard Gabor approach}

The proposed approach, although principally similar to the one proposed by Shen et. al. [47], varies in many aspects. The authors in [47] use a bank of gabor filters with eight different orientations and one constant frequency, and convolve the image with this bank of gabor filters. Their block-based technique is based on the criteria that for every block, in presence of a prominent ridge-valley pattern, atleast one or several gabor filters will match the underlying fingerprint pattern. This will result in good response for some filters and poor response for the others. In the case of a poor quality or a background block, none of the gabor filters would result in a good response. Thus, on basis of the standard deviation across these filter responses, a block could be classified as being poor or good. 
Gabor filters capture both the local and global features within a fingerprint. Eight different directions are required to completely capture the local ridge characteristics in a fingerprint while only four directions are required to capture the global configuration [5], [57]. In our formulation we wish to analyze the local information, hence we use eight different orientations. Also in fingerprints, even though the ridges and valleys are parallel to each other, the inter-ridge distance varies across the whole fingerprint and also across fingerprints. On basis of this observation, gabor filters with five different frequencies are utilized. A pixel-based approach is implemented instead of a block-based approach to check the contribution of each pixel towards the quality of the image. Thus, each pixel is analyzed within its corresponding neighborhood, defined by the size of the gabor filter mask. In presence of a good quality neighborhood, one of the filters produce a better response than the rest as the filter frequency and orientation matches the underlying ridge-valley pattern. While for a poor quality neighborhood, none of the filters from the filterbank produce a good response due to the lack of underlying ridge-valley pattern. Hence, the maximum response at all the pixels across all the filters is recorded.

In the proposed formulation, parameters of the gabor filter are: $m=8, n=5, \sigma_{x}=4, \sigma_{y}$ $=4, w=20$ and $f_{t}$ takes on five $(n=5)$ different values $[1 / 6,1 / 7,1 / 8,1 / 9,1 / 10]$. Thus, the orientations of the gabor filter vary in the steps of $22.5^{\circ}\left(180^{\circ} / \mathrm{m}\right)$ and the inter-ridge distances vary between 6 and 10 pixels in steps of one. For every image at every pixel, $m \times n=8 \times 5=$ 40 gabor features are computed. Fig. 3.2 provides an illustration of the gabor filters with same orientation and five different frequencies.

\subsubsection{Gabor-based Quality Classification}

The gabor-based quality classification aids in classifying images into good, dry and smudged images. The most prominent steps are foreground segmentation and pixel-based gabor response analysis, after the segmented foreground is convolved with the gabor filterbank. This process is summarized in the flow chart shown in Fig. 3.3 .

\section{Foreground Segmentation}

The gabor response is only computed in the foreground and, hence, foreground segmentation is required. Foreground region corresponds to the area containing ridges and valleys, which is the area of interest. The background region corresponds to region outside the borders of the 


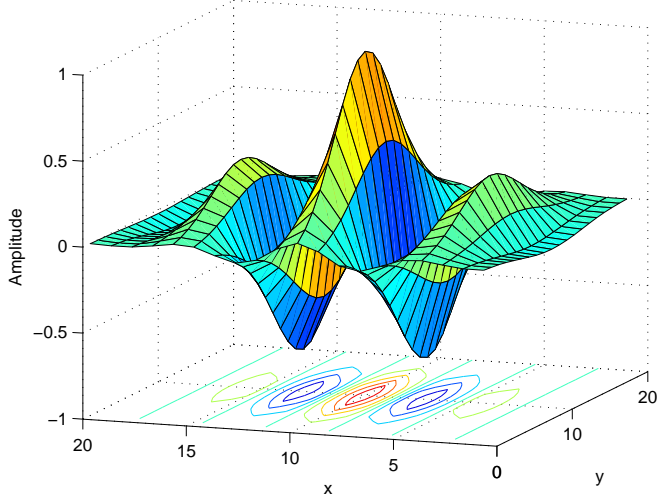

(a)

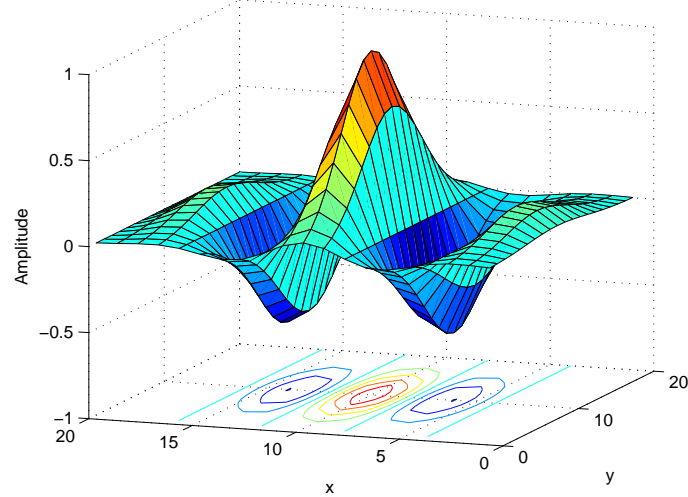

(c)

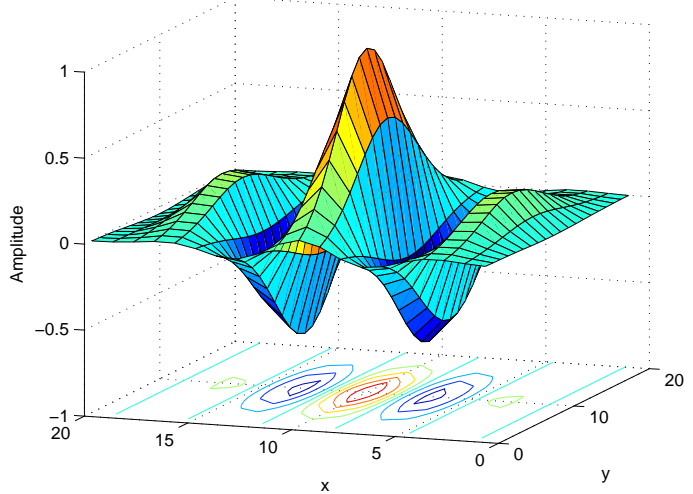

(b)

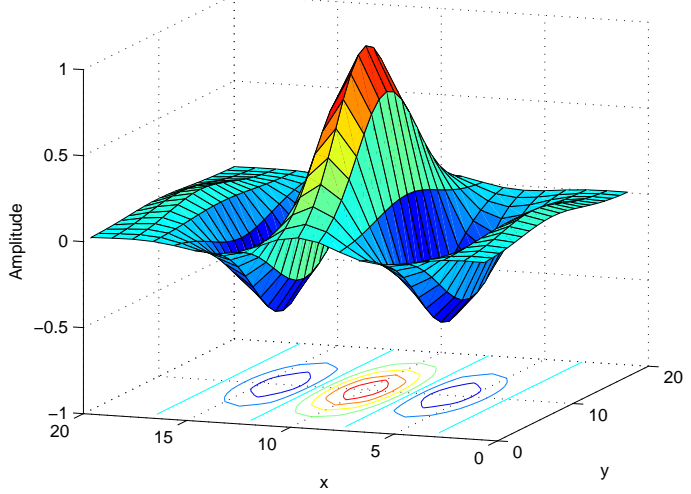

(d)

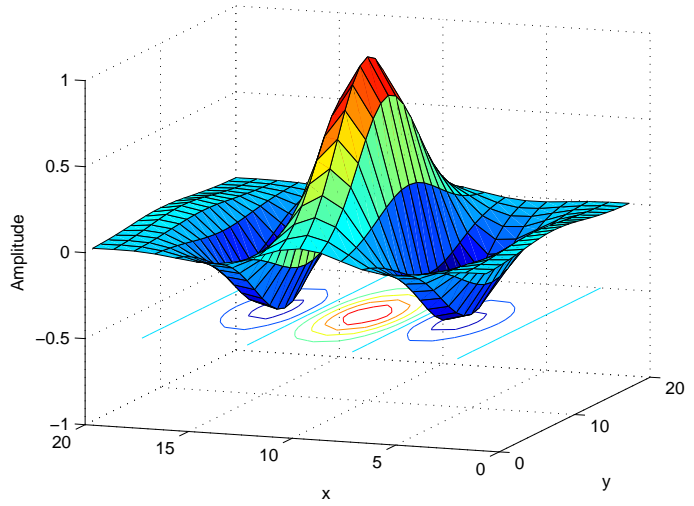

(e)

Figure 3.2: Gabor filters with the same orientation but different inter-ridge distances (frequencies) (a) 6 pixels, (b) 7 pixels, (c) 8 pixels, (d) 9 pixels, (e) 10 pixels. 


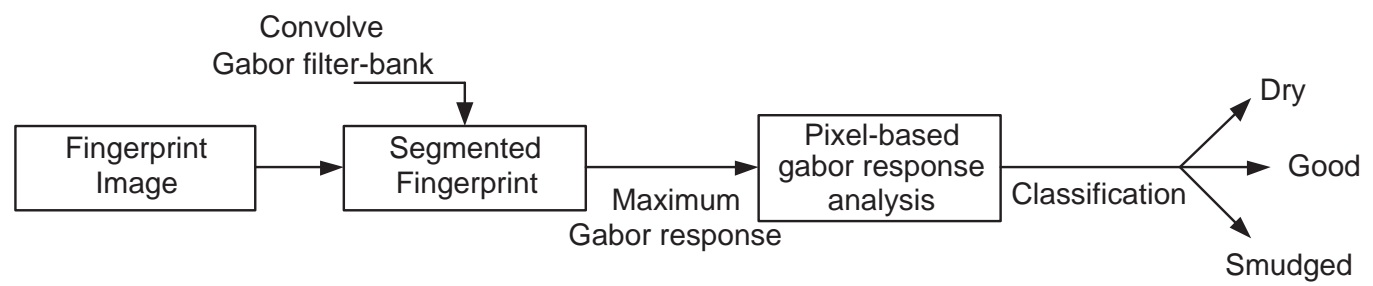

Figure 3.3: Flowchart for gabor-based quality classification approach

foreground area, which does not contain any valid fingerprint information (ridge-valley structure). A block-based variance thresholding approach is used for segmentation as the background greyscale variance is low in comparison with the foreground grey-scale variance. In the first step, the image is divided into blocks and the variance of the grey-scale intensities within each block of the image is calculated. The blocks are classified as foreground or background depending on a global threshold. If the variance is less than the global threshold, then the block is labeled as background block. A block is labeled as foreground block, if the variance is greater than the global threshold. In the proposed scheme, global threshold is defined as 0.1. The grey-level variance for a block of size $W \times W$ is defined as:

$$
\operatorname{var}(k)=\frac{1}{W^{2}} \sum_{i=0}^{W-1} \sum_{j=0}^{W-1}(I(i, j)-M(k))^{2}
$$

where, $\operatorname{var}(k)$ is the variance of the block $k, I(i, j)$ is the grey-level value at pixel $(i, j)$ and $M(k)$ is the mean of the grey-level intensities in block $k$.

Within the detected foreground region, the above procedure may label a few blocks as background blocks. These blocks may actually correspond to dry or smudged areas within the foreground and are considered as misclassified blocks. Hence, morphological operators are used to fill holes and detect the region with largest area, which would correspond to the true fingerprint foreground area. An illustration of a few segmented fingerprints, using the above mentioned variance thresholding approach, along with original fingerprints is shown in Fig. 3.4 .

\section{Pixel-based Gabor response analysis}

The gabor filterbank consisting of 40 different filters is convolved with each fingerprint image (refer Eq. 3.5). The maximum response at each pixel $z(i, j)$ within the segmented foreground 


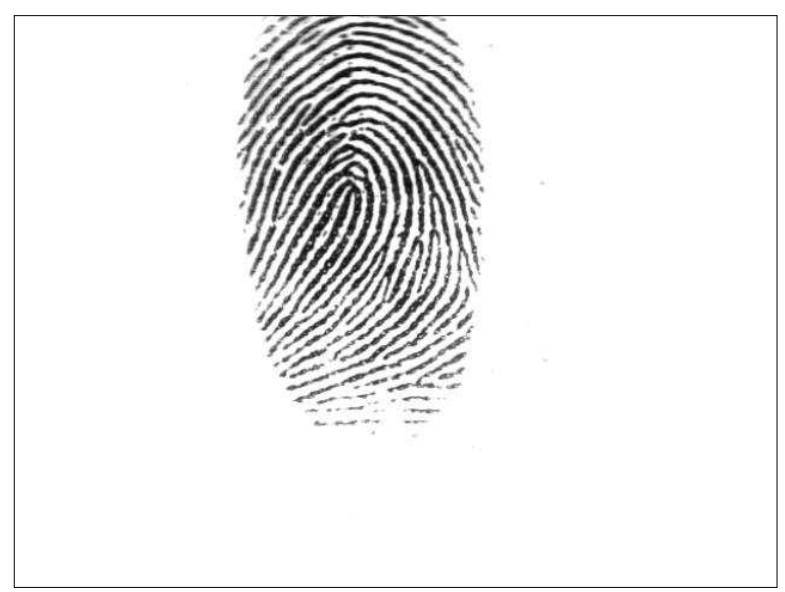

(a)

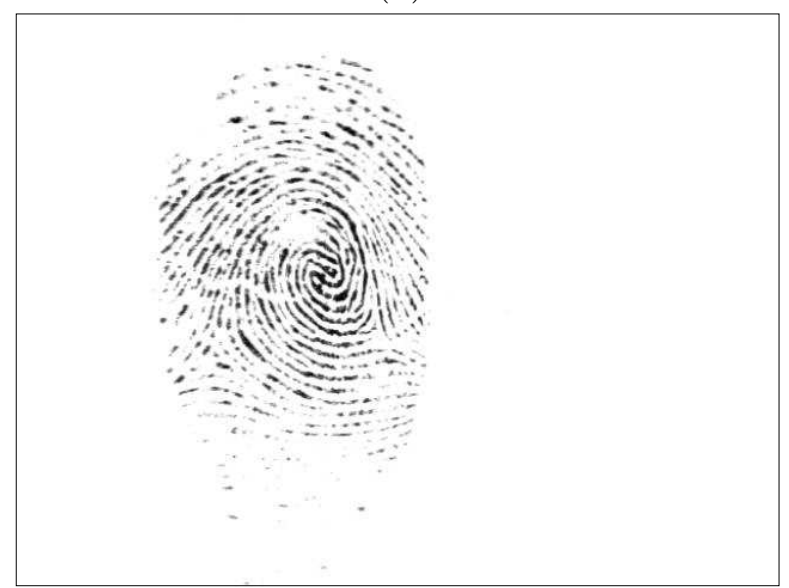

(c)

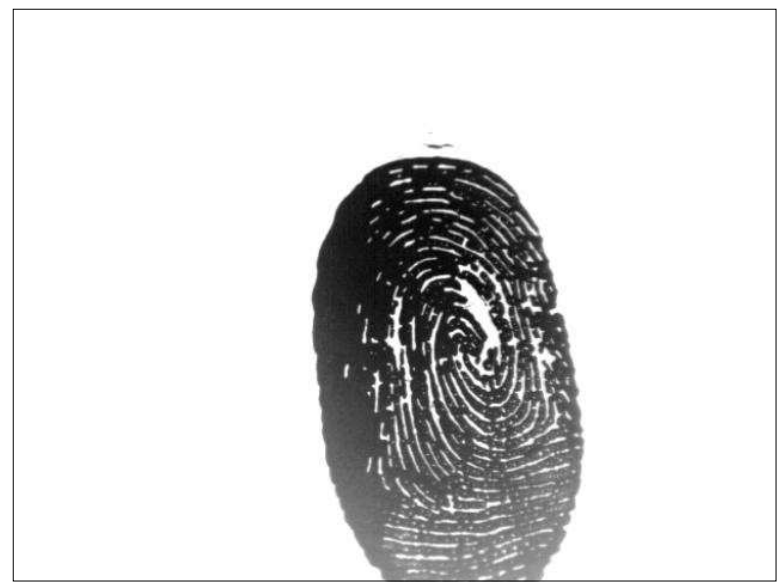

(e)

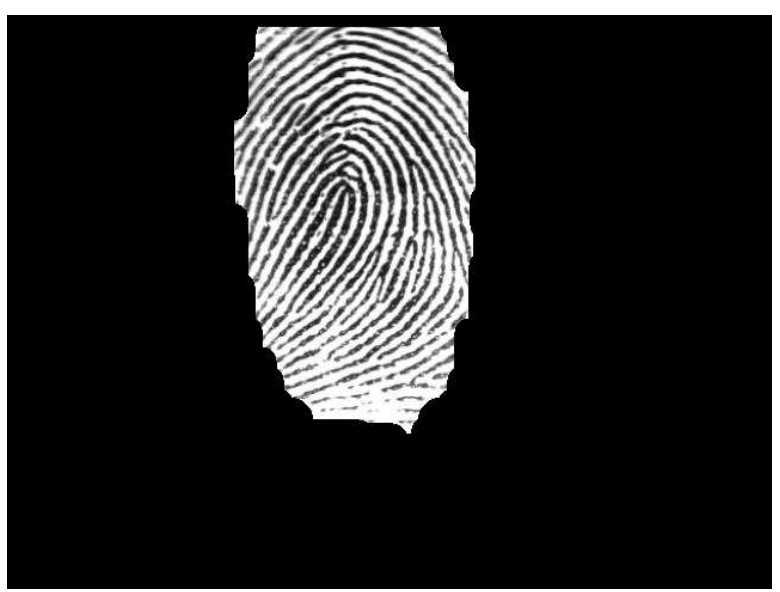

(b)

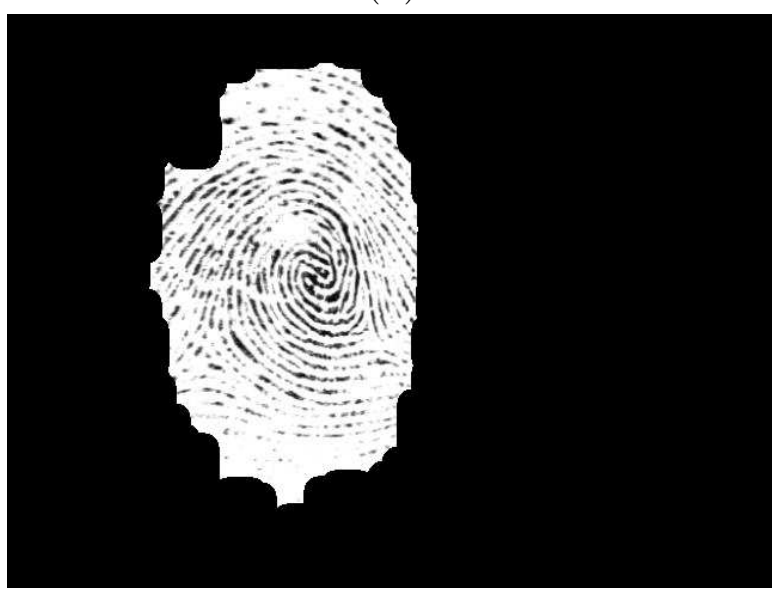

(d)

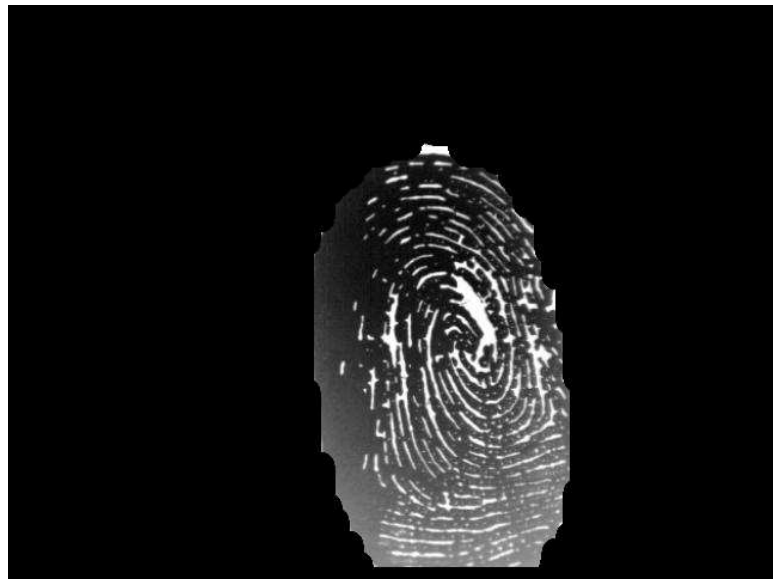

(f)

Figure 3.4: Fingerprint foreground segmentation of (a) Good image (c) Dry image (e) Smudged image and their corresponding segmented images (b), (d), (f), respectively. 
area $(F)$ is recorded, as shown in Eq. 3.7.

$$
z(i, j)=\max _{(i, j) \in F, k=1 . . m, t=1 . . n}\left\{g\left(i, j, \theta_{k}, f_{t}, \sigma_{x}, \sigma_{y}\right)\right\}
$$

In the work reported by [47], [48], thresholds are defined for the gabor responses to associate a quality with each block. Similarly in this scheme, thresholds are defined to classify the pixel into good, dry or smudged quality depending on the neighboring region. In a good quality region, since the underlying ridge valley pattern is prominent, a good response is observed across atleast one filter. While for a poor quality region, a poor or moderate response is observed across all the filters. Hence, the maximum gabor response values across good quality regions and poor quality regions differ significantly. This observation helps in distinguishing between the good and the poor quality pixels but not in classifying the poor quality pixels into dry and smudged.

Another aspect which should not be overlooked is the fact that a gabor response is a measure of not only the filter matching the underlying pattern but also the pixel intensity as shown in Eq. 3.5. Poor quality pixels can be classified into dry and smudged on the basis of these pixel intensity-based responses. Dry areas should ideally comprise of pixel intensities close to 255 (for a 256 grey-scale image) while smudged areas should comprise of pixel intensities close to 0. But this ideal scenario does not always exist and hence variations in the grey-level intensities close to 255 and 0 are observed. These variations propagate into the gabor responses and thus thresholds can be defined for segregating dry quality pixels from the smudged quality pixels.

Threshold determination - All the results with the intermediate steps for the gabor-based quality classification approach, for a good-quality fingerprint image are shown in Fig. 3.5. The histogram of gabor pixel responses is a good indicator of the percentage of pixels contributing towards good, dry and smudged qualities. Fig. 3.6, 3.7 and 3.8 reveal the variation in the histogram of gabor pixel responses for good, dry and smudged quality images. The maximum gabor response image for each of these fingerprints is also displayed. On basis of the significant difference in the histograms of the different quality images, two different threshold values of $\lambda_{1}$ and $\lambda_{2}$ are defined to analyze the contribution of pixels towards the global image quality. Percentage of pixels within the foreground $(F)$ contributing towards a gabor response value that is: (a) less than $\lambda_{1}$ (b) between $\lambda_{1}$ and $\lambda_{2}$ and (c) above $\lambda_{2}$, are used for global quality analysis. 
These percentages are defined as (a) $X_{\lambda_{1}}$, (b) $X_{\lambda_{1} \lambda_{2}}$ and (c) $X_{\lambda_{2}}$, respectively (refer Eq. 3.8).

$$
\begin{aligned}
X_{\lambda_{1}} & =\frac{\left(\sum \sum_{i=1, j=1,(i, j) \in F}^{i=M, j=N} I\left(z(i, j) \leq \lambda_{1}\right)\right)}{\left(\sum \sum_{i=1, j=1,(i, j) \in F}^{i=M, j=N} 1\right)} * 100 \\
X_{\lambda_{1} \lambda_{2}} & =\frac{\left(\sum \sum_{i=1, j=1,(i, j) \in F}^{i=M, j=N} I\left(\lambda_{1}<z(i, j) \leq \lambda_{2}\right)\right)}{\left(\sum \sum_{i=1, j=1,(i, j) \in F}^{i=M, j=N} 1\right)} * 100 \\
X_{\lambda_{2}} & =\frac{\left(\sum \sum_{i=1, j=1,(i, j) \in F}^{i=M, j=N} I\left(z(i, j) \geq \lambda_{2}\right)\right)}{\left(\sum \sum_{i=1, j=1,(i, j) \in F}^{i=M, j=N} 1\right)} * 100
\end{aligned}
$$

where, $I(e)=1$ if $e$ is true.

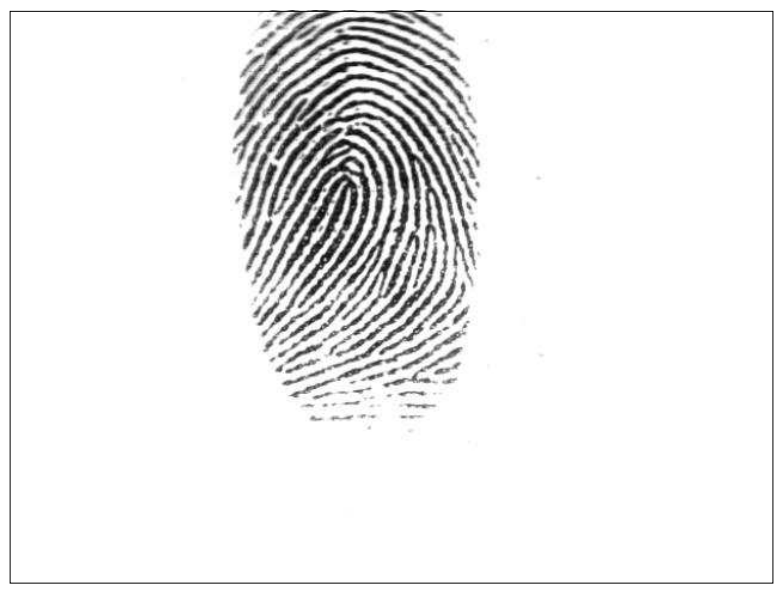

(a)

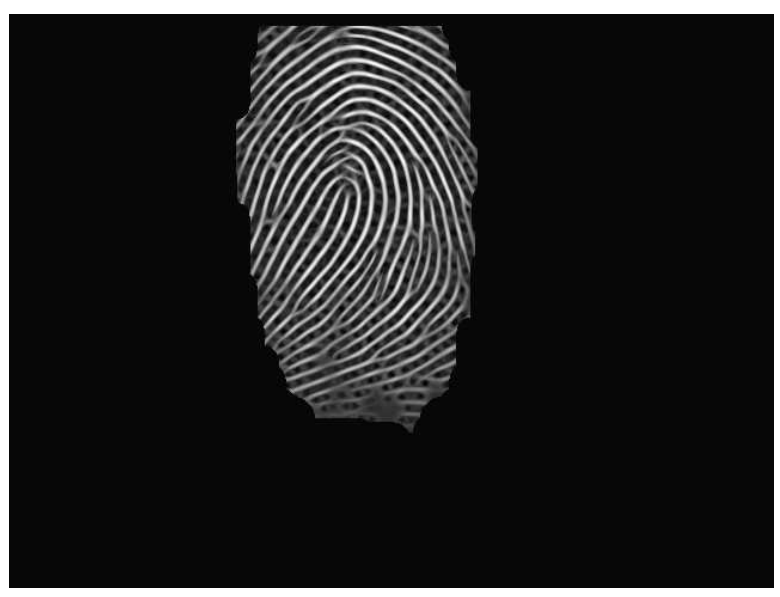

(c)

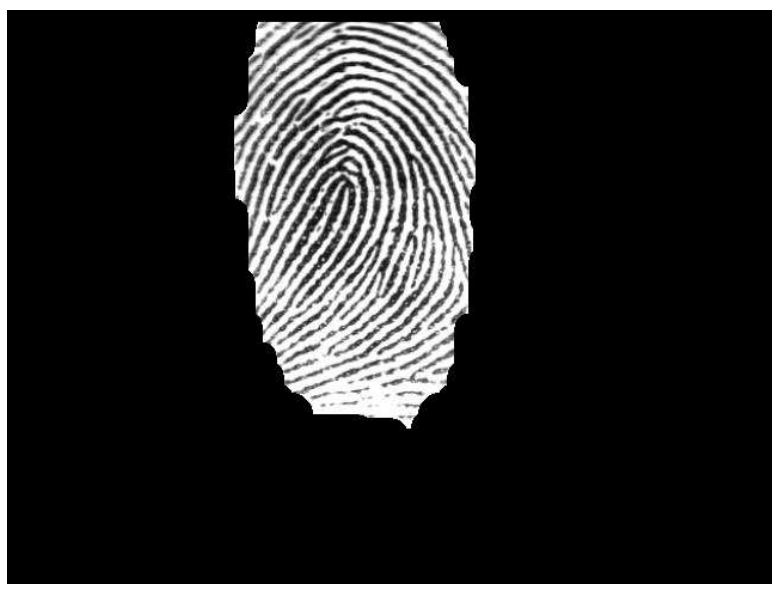

(b)

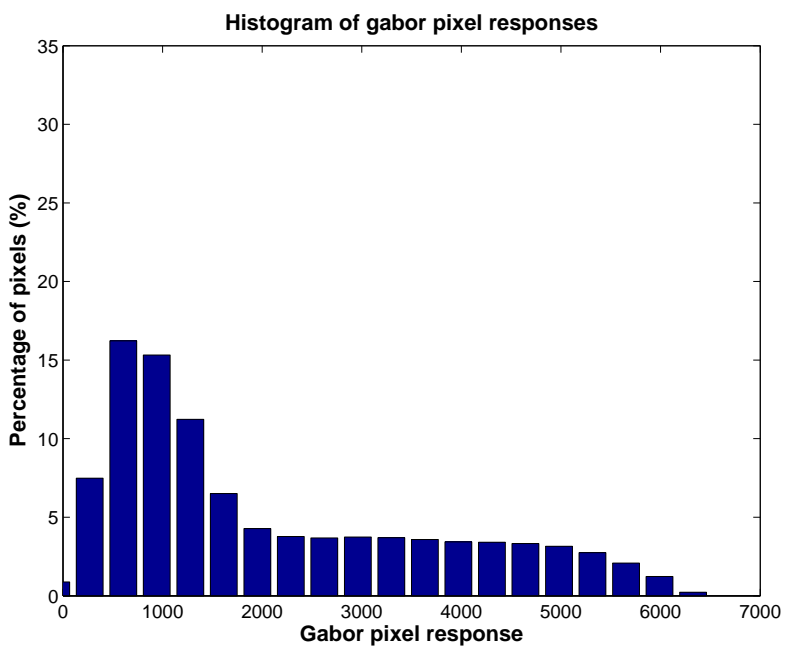

(d)

Figure 3.5: Intermediate results for the gabor-based classification approach (a) Original image (b) Segmented image (c) Maximum gabor response image (d) Histogram of gabor pixel responses. 


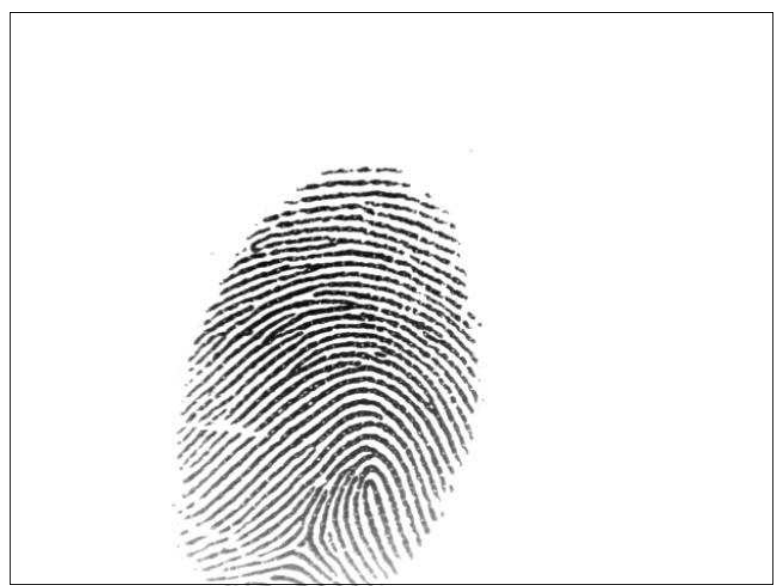

(a)

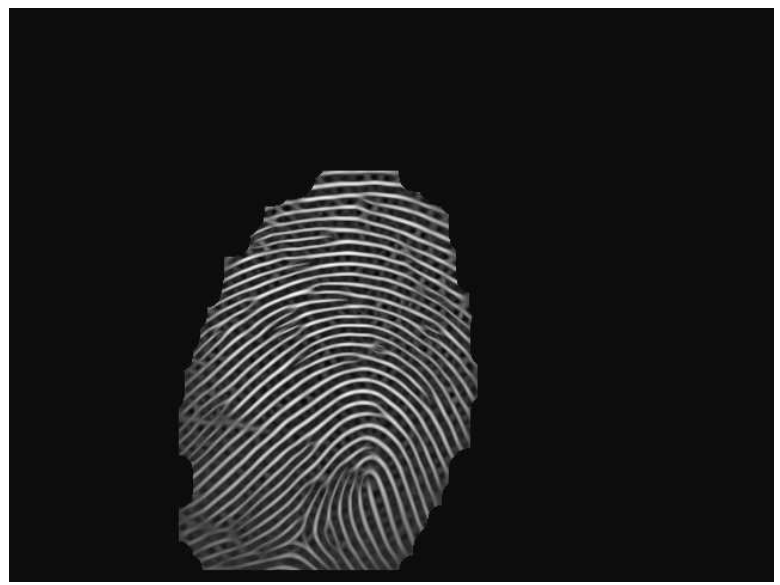

(c)

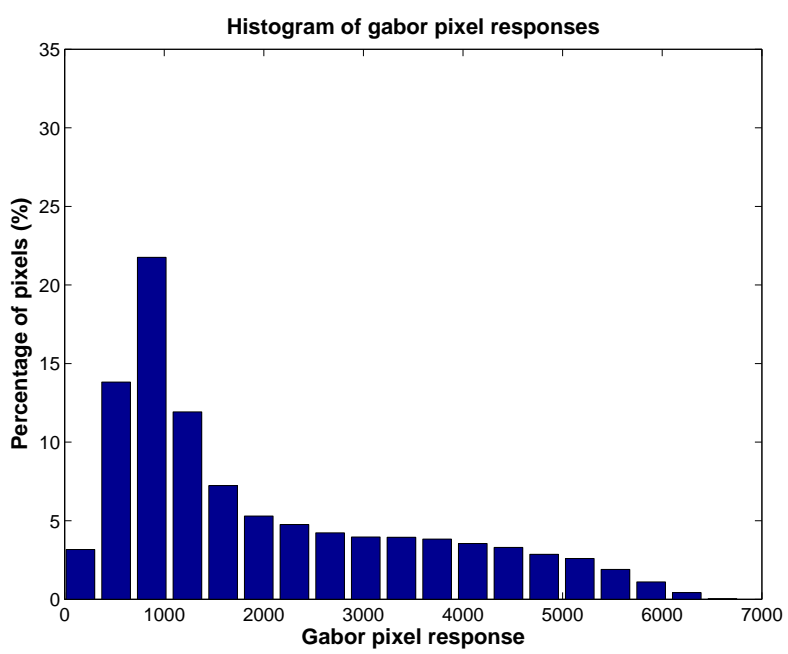

(e)

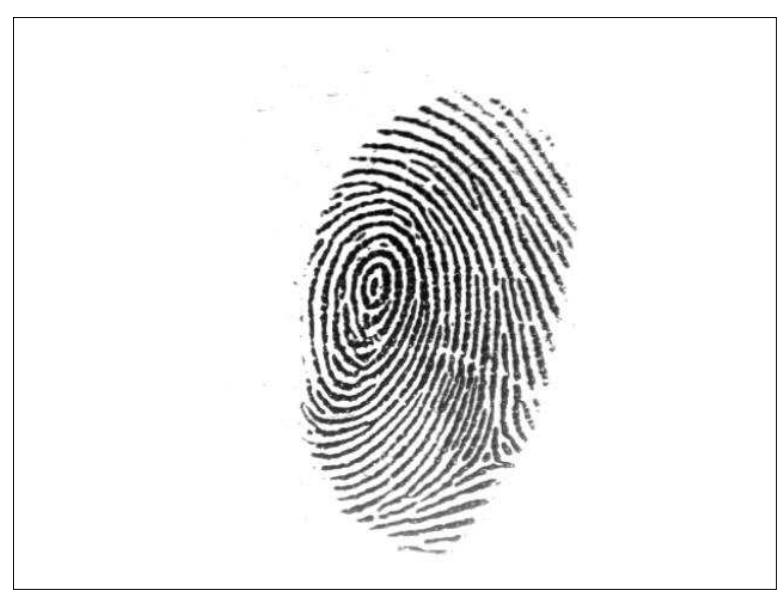

(b)

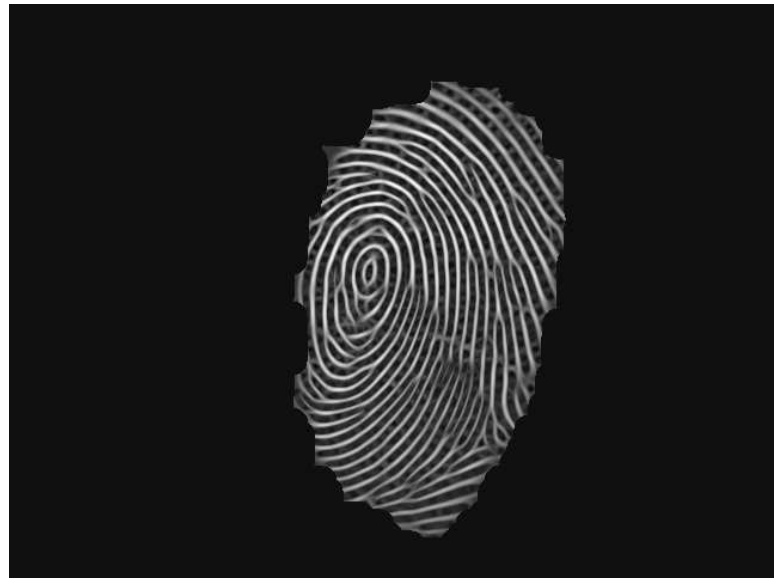

(d)

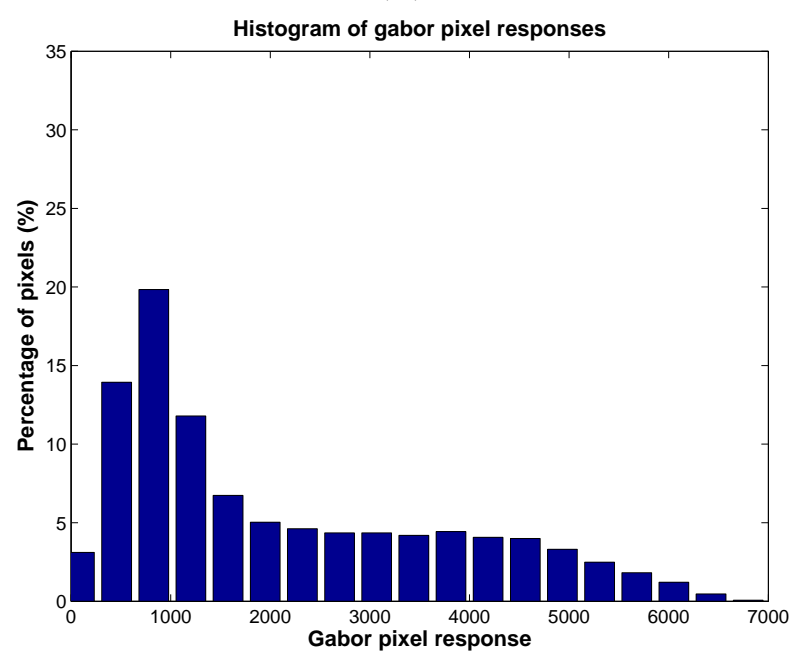

(f)

Figure 3.6: Pixel-based gabor response of good quality images shown in (a) good 1 and (b) $\operatorname{good}_{2}$. (c) and (d) show the corresponding maximum gabor response images. Histogram of gabor pixel responses are shown in (e) and (f). 


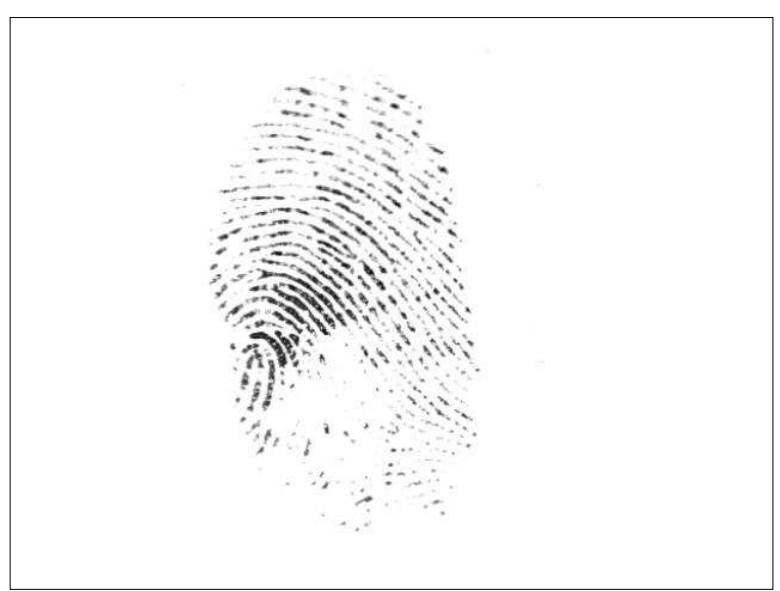

(a)

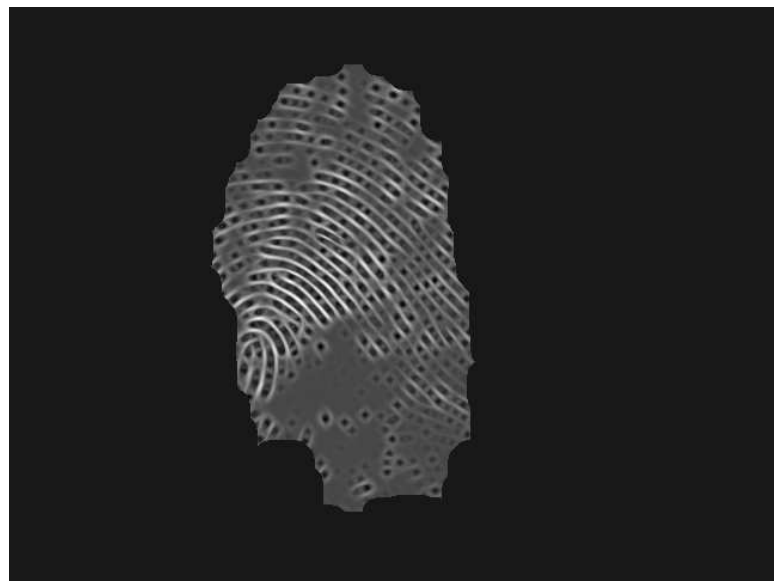

(c)

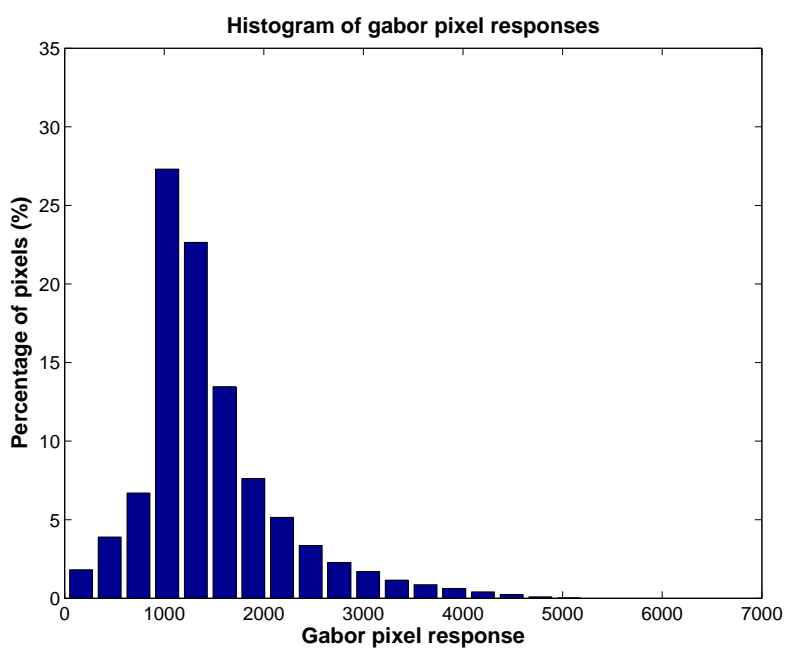

(e)

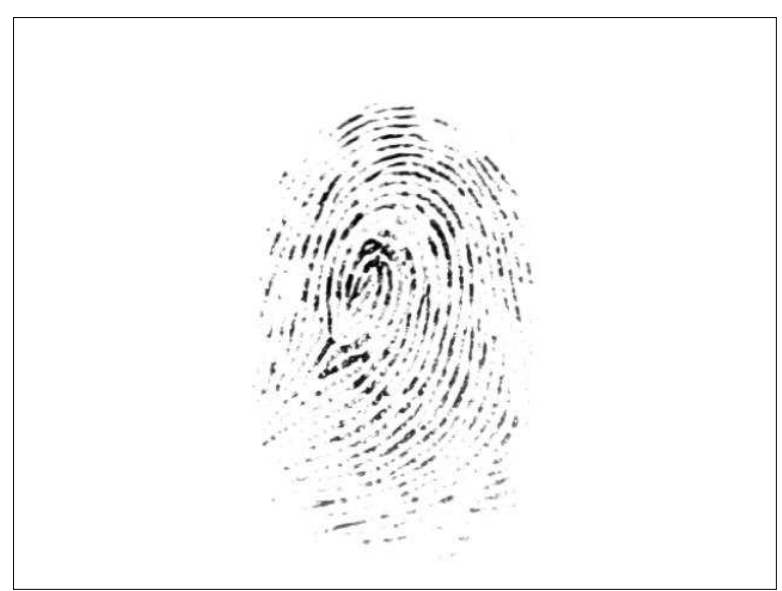

(b)

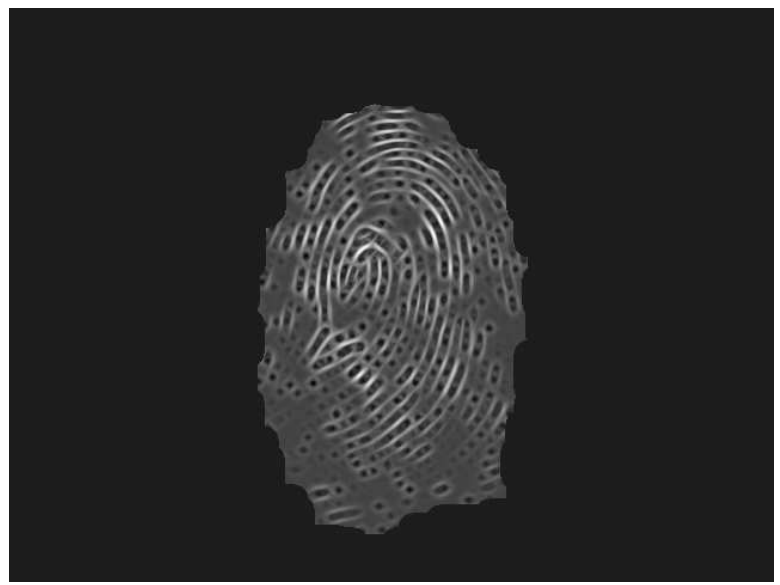

(d)

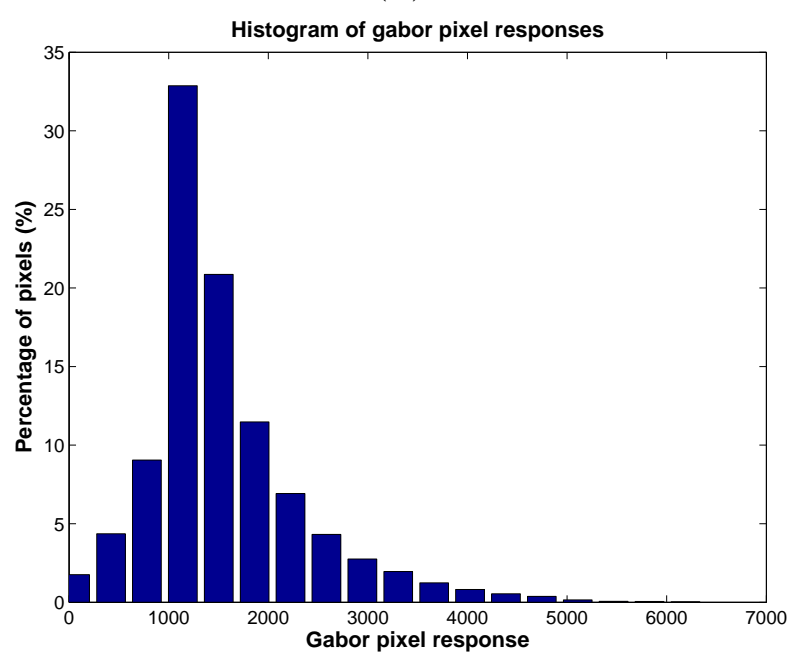

(f)

Figure 3.7: Pixel-based gabor response of $d r y$ quality images shown in (a) $d r y_{1}$ and (b) $d r y_{2}$. (c) and (d) show the corresponding maximum gabor response images. Histogram of gabor pixel responses are shown in (e) and (f). 


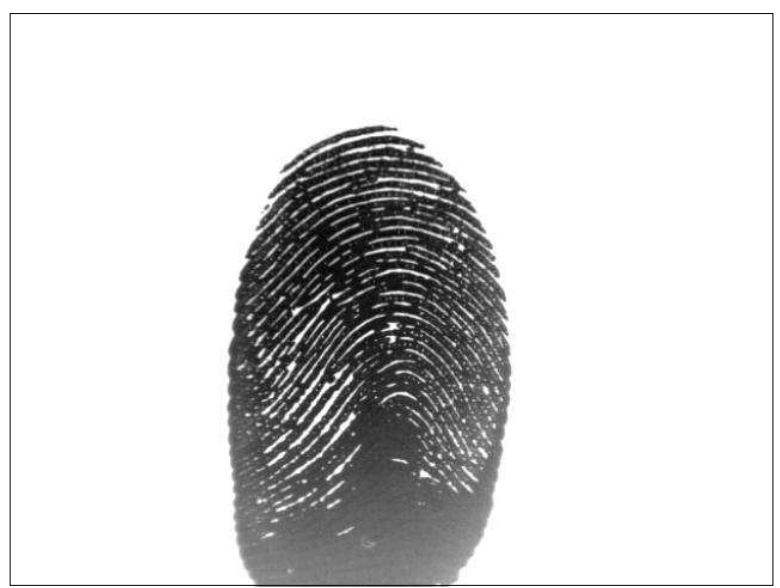

(a)

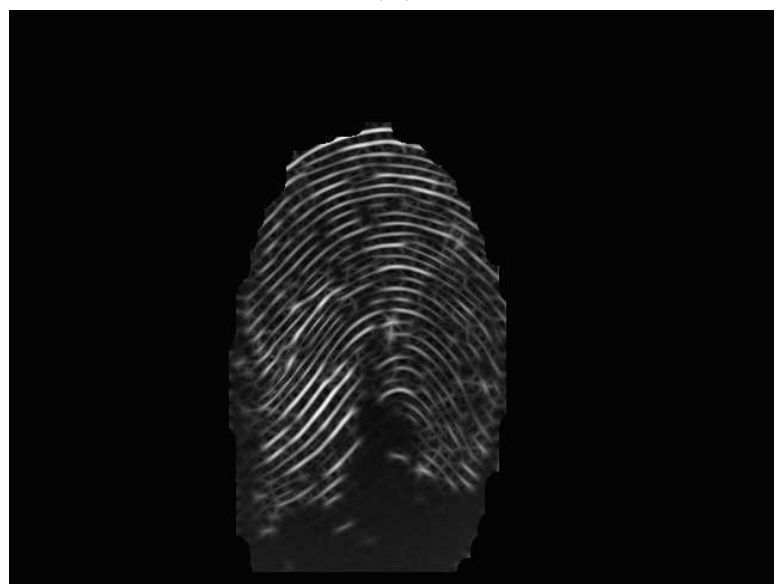

(c)

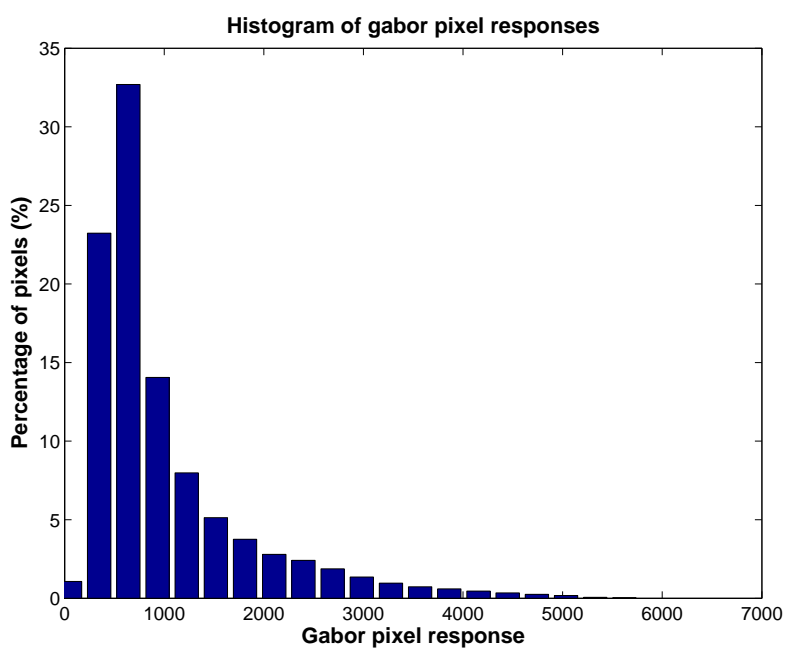

(e)

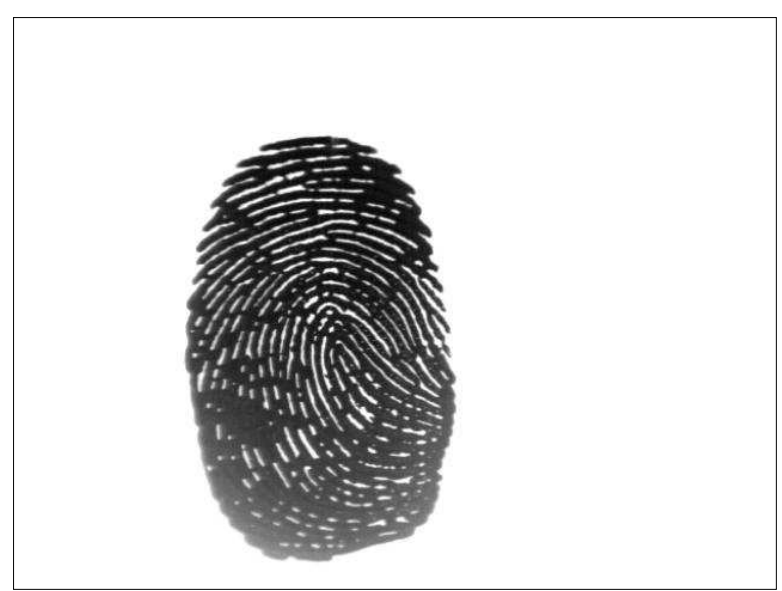

(b)

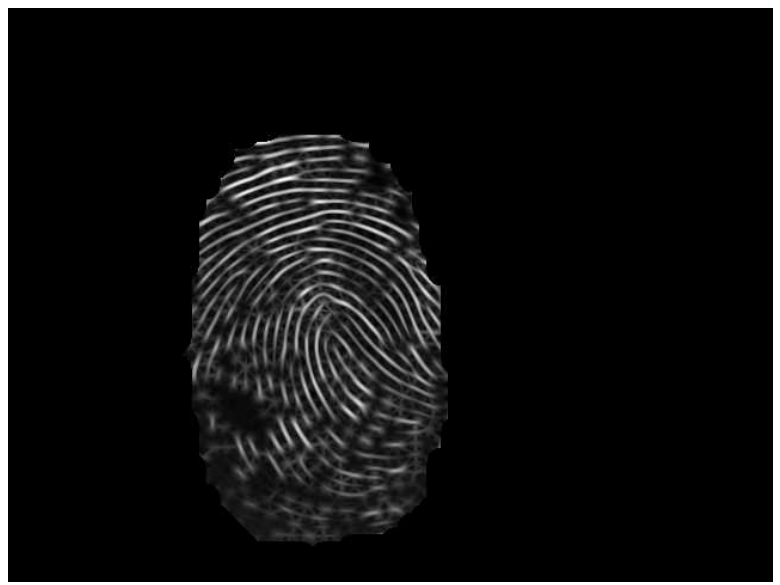

(d)

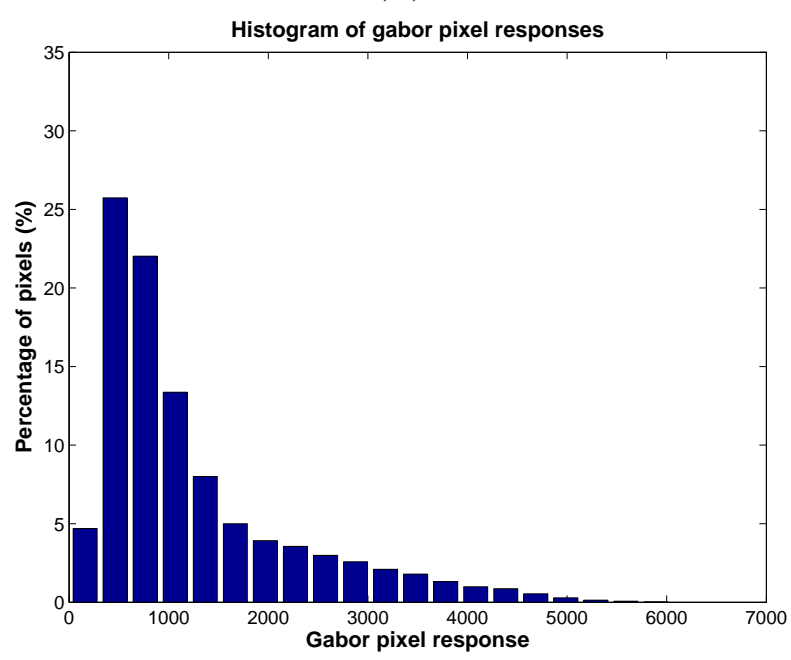

(f)

Figure 3.8: Pixel-based gabor response of smudged quality images shown in (a) smudged smd $_{1}$

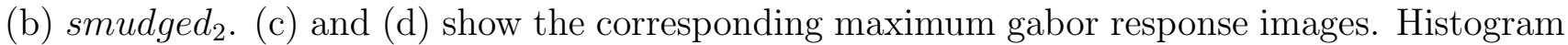
of gabor pixel responses are shown in (e) and (f). 
In the current formulation, values of $\lambda_{1}$ and $\lambda_{2}$ are selected as 1000 and 2000, respectively. These thresholds are based on the observation that the histogram of good quality images reveal a high percentage of pixels contributing towards a better gabor pixel response (above a value of $\lambda_{2}$ ). For a dry quality image, a peak (high percentage of pixels) is observed between the response values of $\lambda_{1}$ and $\lambda_{2}$. This actually corresponds to the 'near 255' pixel intensities in the original image. In the case of a smudged quality image, large percentage of pixels are observed below the $\lambda_{1}$ response value, which relate to the 'near 0' pixel intensity in the original image.

\section{Classification}

The percentages of pixels defined by these thresholds for each of the images displayed in Fig. 3.6, 3.7 and 3.8 are reported in the Table 3.1. For the dry images, high pixel percentages $(65.54 \%$

\begin{tabular}{|l|l|l|l|l|}
\hline No. & Image quality & $X_{\lambda_{1}} \%$ & $X_{\lambda_{1} \lambda_{2}} \%$ & $X_{\lambda_{2}} \%$ \\
\hline \hline 1 & good $_{1}$ & 35.87 & 25.55 & 38.26 \\
\hline \hline 2 & good $_{2}$ & 35.89 & 22.70 & 41.05 \\
\hline \hline 3 & dry $_{1}$ & 17.18 & 65.54 & 16.74 \\
\hline \hline 4 & dry $_{2}$ & 16.37 & 62.57 & 20.29 \\
\hline \hline 5 & smudged $_{1}$ & 68.00 & 20.36 & 11.64 \\
\hline \hline 6 & smudged $_{2}$ & 57.01 & 24.22 & 18.77 \\
\hline
\end{tabular}

Table 3.1: Percentage of pixels defined by the thresholds $\lambda_{1}$ and $\lambda_{2}$, used for image quality classification.

and $62.57 \%)$ are observed for $X_{\lambda_{1} \lambda_{2}}$, whereas for smudged images high percentages $(68.00 \%$ and $57.01 \%$ ) are observed below $X_{\lambda_{1}}$. For good quality images, the distribution of percentages is pretty even across the three bins created by the thresholds.

On basis of the significant variations observed across the three threshold percentages, good, dry and smudged quality images can be classified. To analyze the potential of this method, each fingerprint image is represented using a 3D feature set comprised of the threshold pixel percentages $\left\{X_{\lambda_{1}}, X_{\lambda_{1} \lambda_{2}}, X_{\lambda_{2}}\right\}$.

\section{Results}

To test the proposed approach, 391 fingerprint images from the FVC 2004 DB1 database ${ }^{2}$ were manually labeled with the associated quality. Visual assessment of these images led to

\footnotetext{
${ }^{2}$ FVC 2004 - DB1 (available @ http://bias.csr.unibo.it/fvc2004/)
} 
Table 3.2: Confusion Matrix based on Naive-Bayes classifier (ten-fold validation)

\begin{tabular}{|c|c|c|c|}
\hline & Good & Dry & Smudged \\
\hline \hline Good & 166 & 1 & 8 \\
\hline \hline Dry & 0 & 122 & 0 \\
\hline \hline Smudged & 1 & 0 & 93 \\
\hline
\end{tabular}

175 good, 122 dry and 94 smudged quality images. The 3D feature set $\left\{X_{\lambda_{1}}, X_{\lambda_{1} \lambda_{2}}, X_{\lambda_{2}}\right\}$ for each image was computed. Fig. 3.9 displays the 3D plot of these 391 images on basis of these 3D feature sets. Three different prominent clusters are observed in the 3D plot with a slight

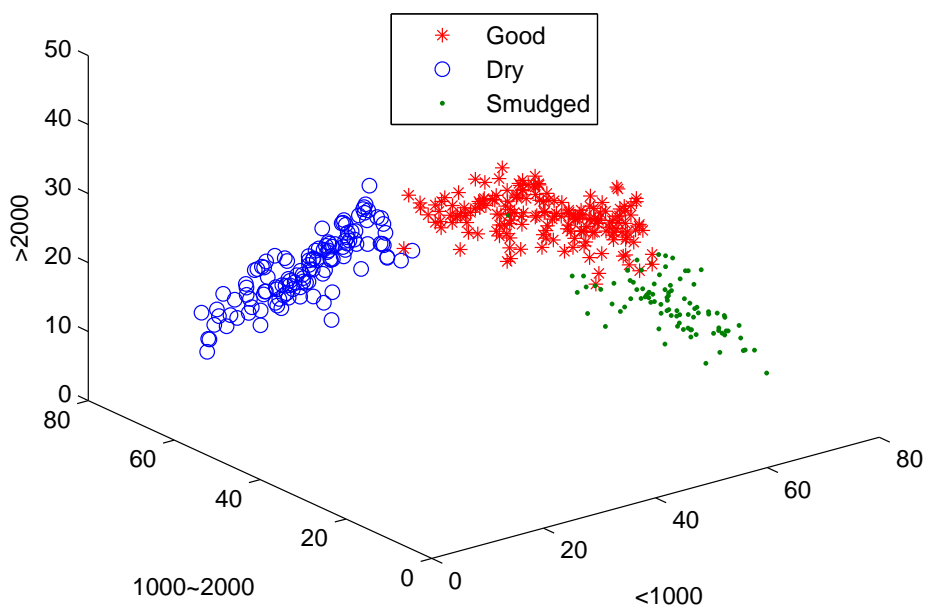

Figure 3.9: Classification of good, dry and smudged quality images using the $\left\{X_{\lambda_{1}}, X_{\lambda_{1} \lambda_{2}}, X_{\lambda_{2}}\right\}$ feature set.

overlap. To estimate the error introduced due to misclassification, the 3D feature set of all the 391 images is passed through a Naive-Bayes classifier ${ }^{3}$. A ten-fold validation approach with a percentage split between the training and testing data of $50 \%$, resulted in a $2.55 \%$ error due to 10 misclassified instances. The confusion matrix as reported by this classifier is provided in Table 3.2 .

\footnotetext{
${ }^{3}$ The Naives-Bayes classifier implemented in WEKA was utilized. WEKA is a collection of machine learning algorithms for data mining tasks available at http://www.cs.waikato.ac.nz/ml/weka/
} 


\subsubsection{Summary and future work}

A novel approach for classifying images into three different categories: good, dry and smudged quality, has been proposed. Such a categorization would assist in invoking different image processing or matching schemes based on the nature of the input fingerprint image. Also, this approach needs to be tested on a database which contains labeled dry, good and smudged quality images.

\section{Limitations}

The above described approach is a heuristic-based approach. The defined thresholds will vary according to the database in consideration. This undermines the ability of this approach to be used across images from multiple databases.

It is very difficult to define an ideal set of parameters $\left\{X_{\lambda_{1}}, X_{\lambda_{1} \lambda_{2}}, X_{\lambda_{2}}\right\}$ that would aid in the computation of a global image quality measure. Slight variations in frequency or pressure can alter these values.

This approach has an inherent limitation based on the assumption of parallel ridges and valleys exhibiting an ideal sinusoidal-shaped plane wave, in a direction orthogonal to the local orientation. But as shown by Yang et al. [58], this is rarely the case and a large deviation from the sine wave is observed especially in the presence of excessive or less pressure.

\subsection{Incorporating estimated quality into a fingerprint recog- nition system}

It has been reported in the literature, that the presence of poor quality images degrades the performance of a fingerprint recognition system. By incorporating the knowledge of quality into different modules of a fingerprint recognition system, improvement in matching performance has been reported [10], [59], [60].

\subsubsection{Incorporating quality at different stages of the recognition sys- tem}

Knowledge of the information present in the fingerprint image can be used for defining two different types of quality: 
- a global image quality measure

- a quality measure associated with each feature extracted from the fingerprint image

During enrollment, the quality of the fingerprint image 'to be enrolled' can be estimated and only images with 'sufficient quality' enrolled into the database. If a similar criteria is used during the identification or verification phase, reliable matching of fingerprints may be achieved. A study was initiated by Simon-Zorita et. al. [59], 660, which assessed the effect of varying image quality and position variability on a minutiae-based fingerprint verification scheme. A significant improvement in performance was reported when poor quality images with position variabilities were excluded from the matching scheme. An improved performance by removal of poor quality images has also been reported in [10], [16], [43].

The estimated image quality can be used to invoke different enhancement and feature extraction algorithms across images. In [10], Chen et. al. tune the parameters of the enhancement algorithm according to the estimated image quality, to improve the performance of the system.

In most of the matching schemes, features are extracted from the fingerprint images which are later utilized for matching purposes. Hence, methods have been proposed which incorporate the local feature quality into the matching scheme. Chen et. al. [10] use the local minutiae quality to estimate the transformation parameters. During matching, if both the matching minutiae are of high quality, this pair would contribute more to the estimation of transformation parameters. NIST associates a quality or reliability measure with every extracted minutiae. During matching, a option is provided which allows minutiae below a certain quality threshold to be rejected from the matching phase [49]. The knowledge of image quality can be integrated into the matching phase. Some systems can be designed to invoke a slower but more powerful matcher when low-quality samples are observed [61]. In [62], Fierrez-Aguilar et. al. have shown that their ridge-based fingerprint matcher is more robust to image quality degradation than their minutiae-based system for a number of different image quality criteria.

The image qualities can also be introduced into the score formulation. The performance of two different fingerprint matchers based on minutiae and ridge information as well as their score level combination has been investigated under varying fingerprint image quality. An adaptive qualitybased score formulation scheme, which relies on the estimated quality has been proposed [17]. The adaptive quality-based score fusion is based on the assumption that the performance of one of the algorithms drops significantly in comparison with the other, in the presence of image 
quality degradation.

Thus, the global image quality or the feature quality can be utilized by the individual modules of the recognition system. In this work, the feature quality measure is incorporated in the matching module.

\subsubsection{Proposed incorporation of extracted Feature Quality into Match- ing Score Formulation}

We believe that it is important to use the knowledge of the quality of extracted features since they are utilized for matching purposes. Also, as the computed score provides a true representation of the 'quality' of matching, the quality associated with each feature should be incorporated into the score formulation. In a minutiae-based matching scheme, a pair of high quality matching minutiae pair should contribute more as compared to a poor quality matching minutiae pair. But different matching schemes utilize different features for matching purposes. In this section, the potential of incorporating feature quality into the score formulation for two different matchers: a minutiae-based matcher and a ridge-based matcher, is demonstrated.

\section{Minutiae-based matcher}

Each fingerprint is represented by a set of extracted features, namely minutiae points. Each minutiae point is generally characterized by its location and orientation. Matching is accomplished by finding the maximum number of minutiae pairings between the enrolled template and the input minutiae set.

\section{Standard Score formulation -}

In the case of a minutiae-based matcher, the matching score is usually a measure of the number of matching minutiae. If the first fingerprint has $k$ minutiae while the second fingerprint has $r$ minutiae and $l$ is the number of matching minutiae pairs across the two fingerprints, then the score $S$ is given by

$$
S=\frac{l^{2}}{k * r}
$$

Proposed Score formulation -

To incorporate the feature quality into the standard score formulation, the following procedure is adopted: Let $Q_{1}=\left\{q_{11}, q_{12}, q_{13}, \cdots, q_{1 k}\right\}$ be the qualities associated with the minutiae 
$M_{1}=\left\{m_{11}, m_{12}, m_{13}, \cdots, m_{1 k}\right\}$ extracted from the first fingerprint. Also, qualities and minutiae associated with the second fingerprint be given by

$$
Q_{2}=\left\{q_{21}, q_{22}, q_{23}, \cdots, q_{2 r}\right\}, M_{2}=\left\{m_{21}, m_{22}, m_{23}, \cdots, m_{2 r}\right\}
$$

respectively. The proposed score formulation $\left(S_{q}\right)$ can then be represented as

$$
S_{q}=\frac{\sum f\left(q_{1 i}, q_{2 j}\right)^{2}}{\left(\sum_{x=1}^{k} q_{1 x}\right) *\left(\sum_{y=1}^{r} q_{2 y}\right)}
$$

where, $i$ and $j$ are the matching minutiae indices in the first and second fingerprint respectively and $f($.$) is a function which combines the qualities of the matching minutiae. The denominator$ represents the product of the sum of all the minutiae qualities for each fingerprint.

\section{Results -}

In this technique, the feature quality associated with each minutiae is computed by implementing the procedure proposed by NIST [4]. The minutiae quality is computed on basis of local information in the area surrounding the minutiae location (see Section 3.2.3). The matching minutiae records are obtained from the VERIFINGER matcher. The original flowchart of a minutiaebased matching algorithm along with the modified flowchart after incorporating feature quality into the score formulation is shown in Fig. 3.10.

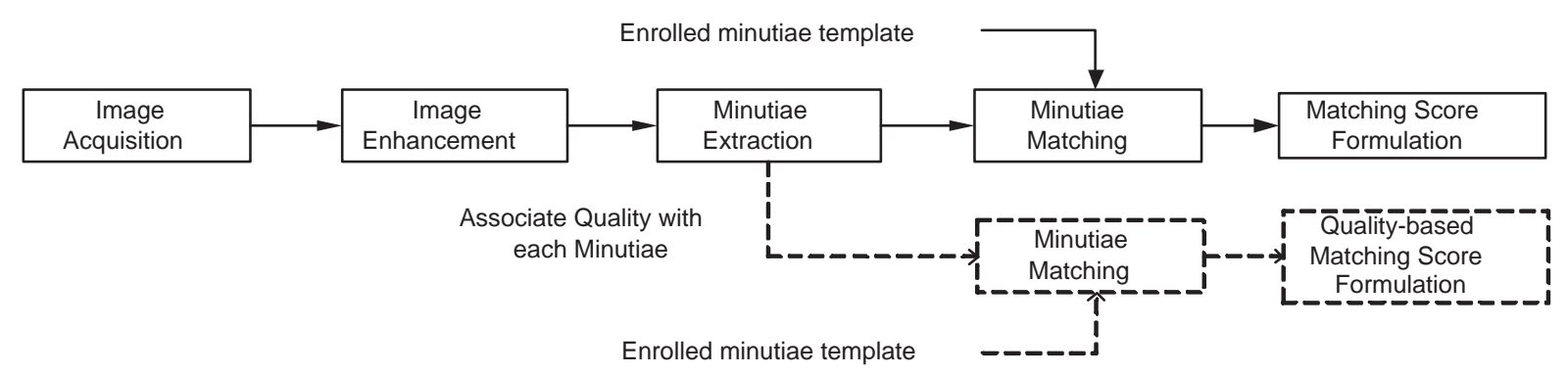

Figure 3.10: Flowchart of a minutiae-based matching algorithm. Modified flowchart for incorporating feature quality into the score formulation is indicated by the dotted lines.

In the proposed formulation, $f($.$) (in Eq. 3.10) is defined as the average of qualities of the$ matching minutiae,

$$
f(.)=\frac{\left(q_{1 i}+q_{2 j}\right)}{2} .
$$

If two poor quality minutiae are matched then their contribution to the matching score will be less as compared to two good quality matching minutiae. Also, two matching minutiae of moderate quality will contribute more than a pair of poor quality matching minutiae. 
The proposed score formulation is compared with the standard score formulation for multiple databases from FVC 2002 and 2004, the results of which are reported in Fig. 3.11. Thus, by incorporating quality into the score formulation, slight improvement in performance is observed.

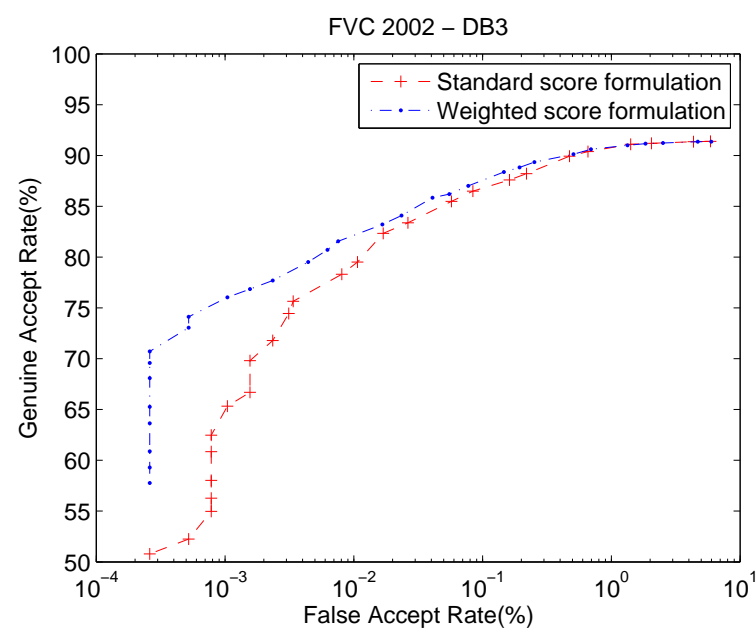

(a)

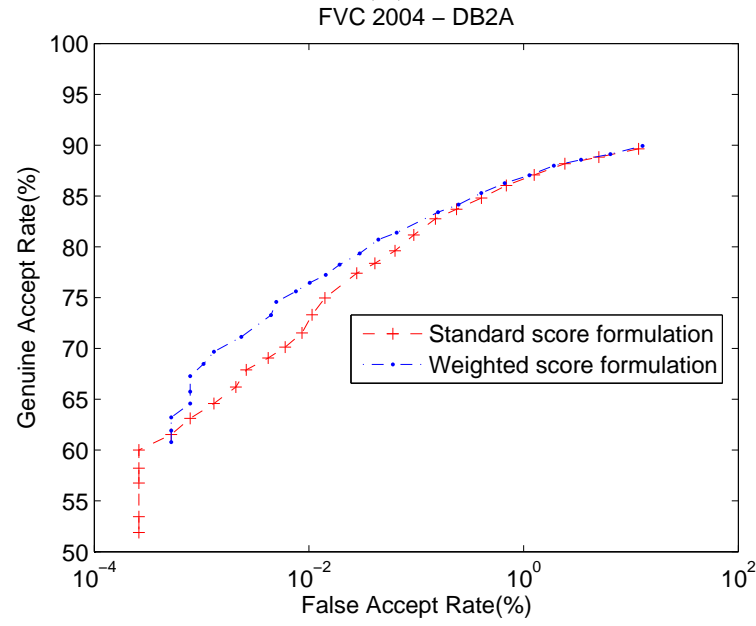

(c)

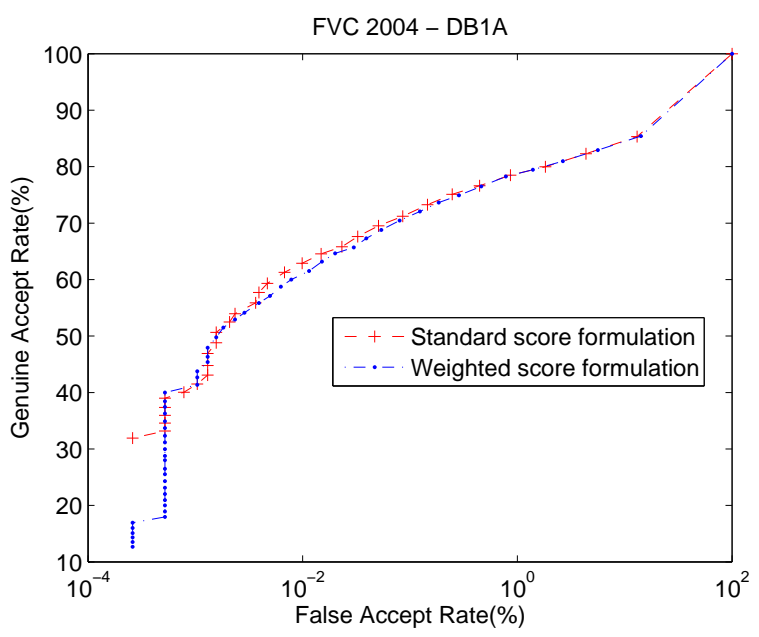

(b)

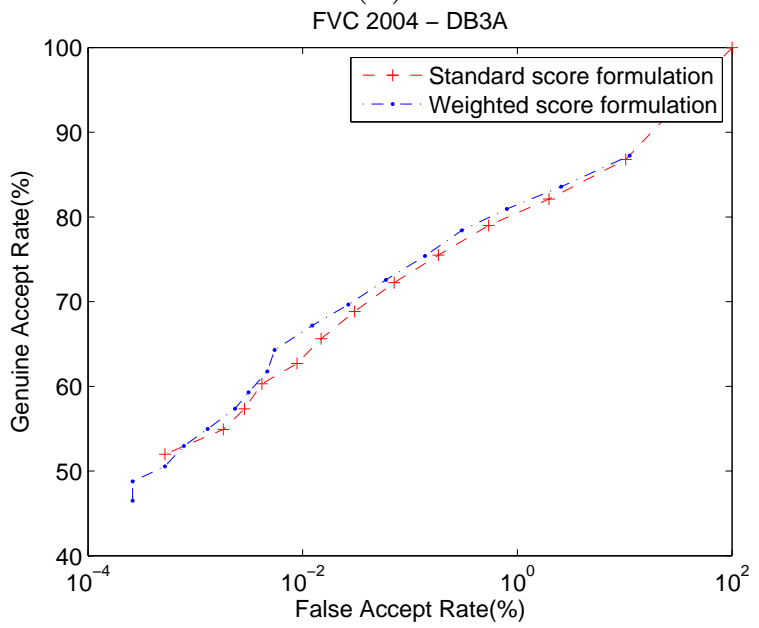

(d)

Figure 3.11: Minutiae-based matcher: Improvement in performance can be observed by comparing the proposed feature quality-based score formulation with the standard score formulation for multiple databases from FVC 2004 and 2002

\section{Feature-based matcher}

The feature-based matcher used to demonstrate the effect of feature quality incorporation into the score formulation, has been proposed by Jain et. al. [5], [56]. In this local texture analysis approach, the fingerprint area of interest is tessellated with respect to the core point. A 
feature vector, called the FingerCode, is generated from the ordered enumeration of the features extracted from the local information, contained in each sector specified by the tessellation. The local texture information in each sector is decomposed into separate channels by using a Gabor filterbank. Matching is performed by computing the Euclidean distance between the fingercodes of the corresponding fingerprints. For more details regarding the feature-based matcher, refer to Appendix C.

Standard Score formulation -

Let $F=\left\{f_{1}, f_{2}, f_{3}, \cdots, f_{k}\right\}$ and $F^{\prime}=\left\{f_{1}^{\prime}, f_{2}^{\prime}, f_{3}^{\prime}, \cdots, f_{k}^{\prime}\right\}$ be the fingercodes of the fingerprints being matched. The score is computed as the euclidean distance across the fingercodes (Eq. 3.11).

$$
D=\sqrt{\sum_{i=1}^{k}\left(f_{i}-f_{i}^{\prime}\right)^{2}}
$$

Proposed Score formulation -

To incorporate the feature quality into the score formulation, the standard score formulation is modified as follows:

$$
D^{\prime}=\frac{\sqrt{\sum_{i=1}^{k}\left(q_{i}+q_{i}^{\prime}\right)\left(f_{i}-f_{i}^{\prime}\right)^{2}}}{\sqrt{\sum_{i=1}^{k}\left(q_{i}+q_{i}^{\prime}\right)}}
$$

where, $Q=\left\{q_{1}, q_{2}, q_{3}, \cdots, q_{k}\right\}$ and $Q^{\prime}=\left\{q_{1}^{\prime}, q_{2}^{\prime}, q_{3}^{\prime}, \cdots, q_{k}^{\prime}\right\}$ are the qualities associated with each of the features.

\section{Results -}

The quality associated with each feature is computed by implementing the procedure proposed by NIST [4]. Here, each feature is associated with a sector within the fingerprint and the same procedure, described in [4], is implemented to associate a quality with each feature. Flowchart of this feature-based matcher along with the modified flowchart after incorporating feature quality into the score formulation is shown in Fig. 3.12 .

The results of the proposed score formulation in comparison with the standard score formulation for multiple databases from FVC 2002 and 2004 are reported in Fig. 3.13. From the performance curves, it can be concluded that by incorporating feature quality into the score formulation improvement in performance can be achieved. 


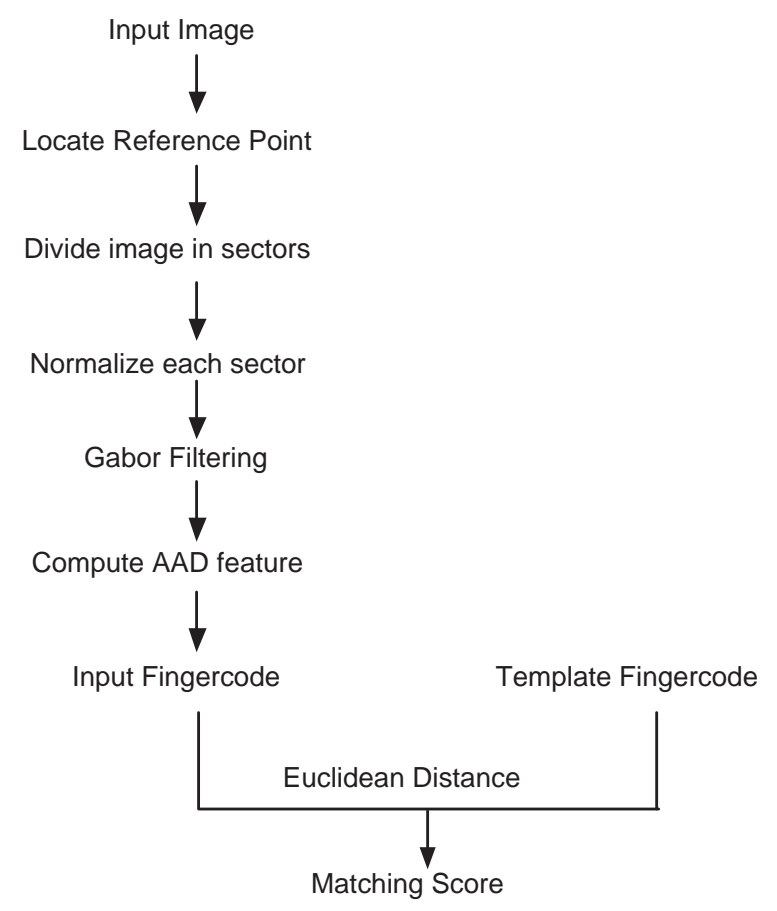

(a)

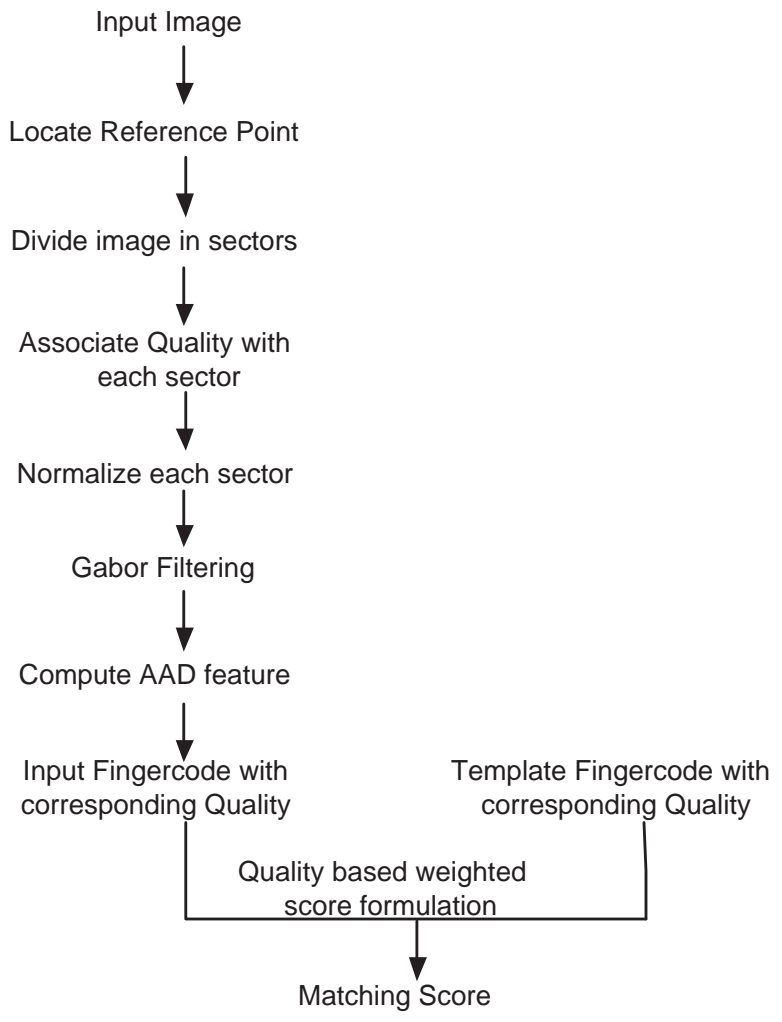

(b)

Figure 3.12: Flowchart of the fingercode based matching algorithm - (a) Original (b) Modified flowchart after incorporating feature quality into the score formulation 


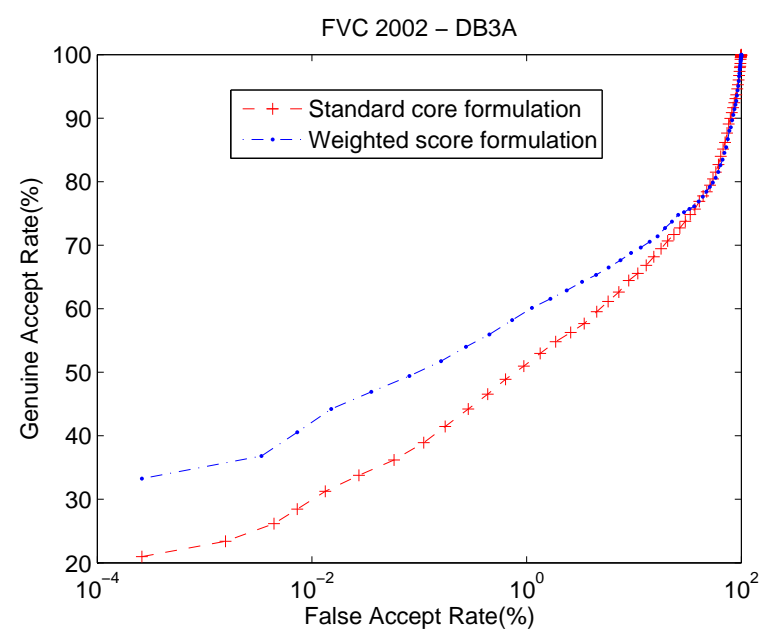

(a)

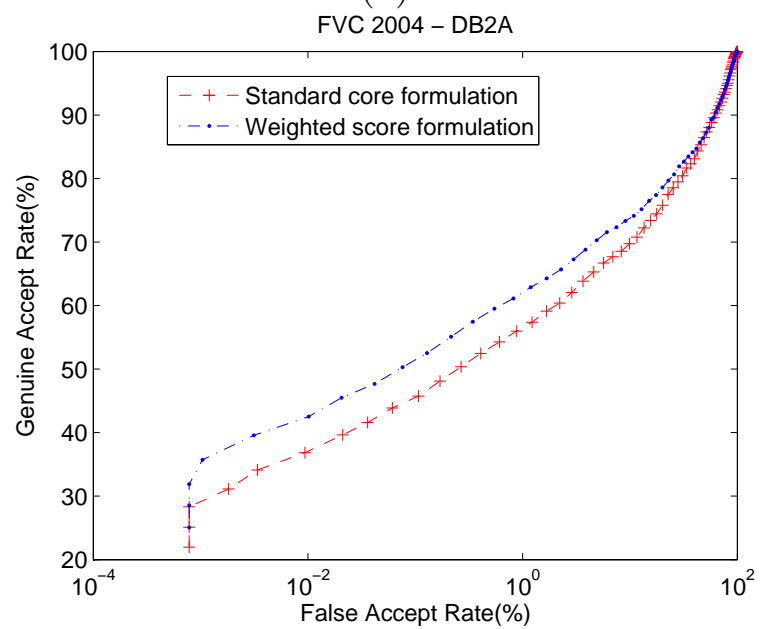

(c)

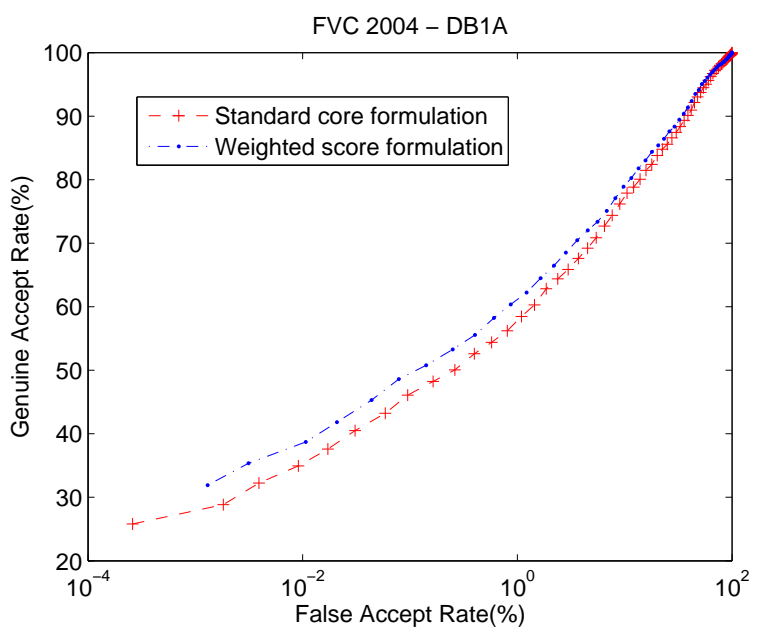

(b)

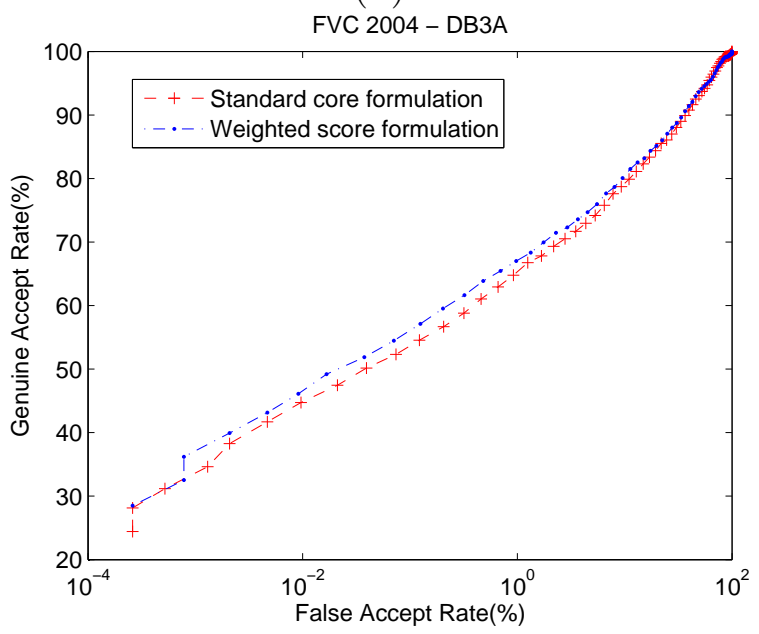

(d)

Figure 3.13: Ridge feature-based matcher: Improvement in performance can be observed by comparing the proposed feature quality-based score formulation with the standard score formulation for multiple databases from FVC 2004 and 2002 


\subsubsection{Conclusion}

From Fig. 3.11 and 3.13, it can be observed that the feature quality-based score formulation leads to an improvement in performance. The potential of such a scheme has been displayed for both, a minutiae-based matcher as well as a ridge feature-based matcher. Such a feature qualitybased score formulation could be useful when dealing with matchers which already exhibit a great performance and slight improvements in performance are desired. More complex score formulations need to be investigated as well. 


\section{Chapter 4}

\section{Summary and Future Work}

\subsection{Sensor Interoperability}

In the proposed model for sensor interoperability, we have demonstrated that a simple nonlinear calibration scheme based on the thin-plate spline model is sufficient to handle variations in minutiae distributions across multiple sensors. The model parameters (average deformation) are computed based on a small representative set of image pairs containing manually established corresponding control points (landmarks). A significant performance improvement is observed when the proposed scheme is utilized to compare fingerprint images originating from two different sensors, viz., optical and solid state capacitive sensors.

The proposed formulation needs to be tested on different matching schemes other than a minutiae-based matching scheme, e.g. a ridge feature-based matcher. In future, more sophisticated calibration grids (similar to camera calibration in computer vision) by imaging rigid finger-like synthetic material with pre-established control points, need to be used. This would avoid issues related to the user-dependent elasticity of the skin. Also a generic calibration model, based on automated control point selection from the representative image pairs, is essential to facilitate interoperability across a wide range of sensors. In the formulation presented in this work, variations introduced in the images and their corresponding features are compensated by defining the solution as a simple transformation function between the extracted features. This approach needs to be evaluated across images acquired using different sensors. To study the feasibility of the proposed approach across multiple sensors, currently data from 3 different sensors (Crossmatch Verifier 300, Secugen Hamster III, Precise 100 AX) is being acquired, in order to 
study the interoperability issues associated with them. Alternate fingerprint matching schemes to address interoperability between minutiae sets obtained from multiple sensors need to be developed as well.

\subsection{Quality}

A novel approach for classifying images into three different categories: good, dry and smudged quality, has been proposed. Such a categorization would assist in invoking different image processing or matching schemes based on the nature of the input fingerprint image.

In this work, we have also shown that the feature quality-based score formulation leads to improvement in performance. The potential of such a scheme has been displayed for both, a minutiae-based matcher as well as a ridge feature-based matcher. Such a feature quality-based score formulation could be useful when dealing with matchers which already exhibit good performance and slight improvements in performance are desired. More complex score formulations that incorporate image quality need to be investigated as well. Also, other factors contributing towards local feature quality need to be assessed. 


\section{Appendix A}

\section{Roll versus Plain Prints: An}

\section{Experimental Study}

\section{A.1 Introduction}

The fingerprint image acquired using a sensor is impacted by several factors including the imaging technology, platen area, sensing resolution, etc. Due to these factors, the fingerprint images obtained using different sensors can be significantly different [63]. Another factor that impacts the photometric and geometric characteristics of a fingerprint image is the acquisition methodology that is employed to procure the fingerprint. For example, contact-based sensors can obtain rolled prints, dab prints or 4 finger slap/plain prints by requiring the subject to interact with the sensor in a particular manner. This results in images whose inherent characteristics are significantly different (see Fig. A.1). In this narrative, the compatibility of rolled prints with slap prints is examined by conducting experiments on the NIST SD 29 database using the NIST fingerprint matching software.

\section{A.2 Background}

A study conducted by Mitretek Systems [1 analyzed the issues affecting the integration of FBI's IAFIS ${ }^{1}$ (that uses 10 rolled prints) with the INS IDENT system ${ }^{2}$ (that uses two flat prints). The study suggested the incorporation of 4 or more dab/flat prints of an individual

\footnotetext{
${ }^{1}$ IAFIS - FBI's Integrated Automated Fingerprint Identification System.

${ }^{2}$ IDENT - Fingerprint matching system used by US-VISIT.
} 


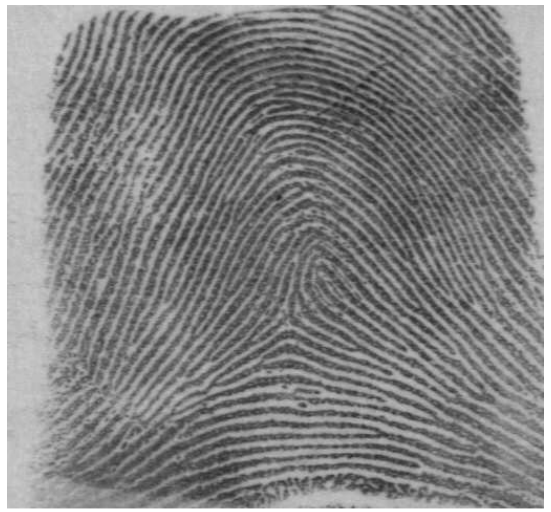

(a)

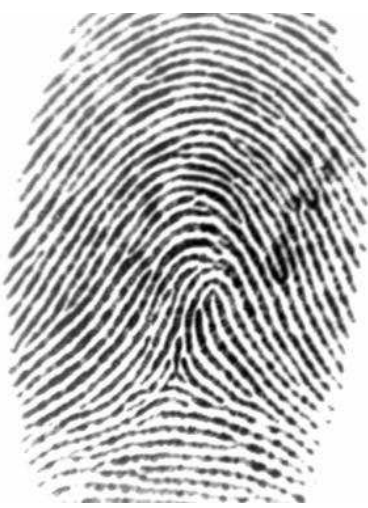

(b)

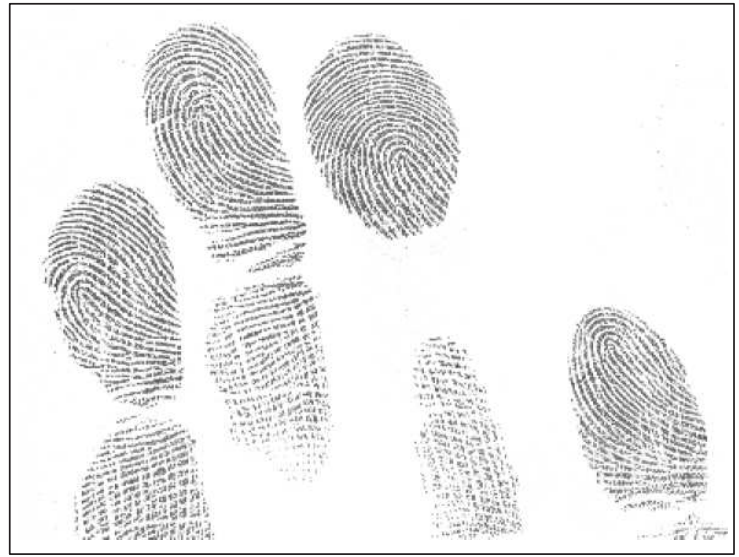

(c)

Figure A.1: Fingerprint images obtained by different acquisition methodologies. (a) Rolled print (from the NIST Special Database 4); (b) Dab print (from the FVC 2002 DB1 Database); and (c) Slap or Plain print [2]. 
into the IDENT system (as opposed to 2-prints) in order to improve the identification accuracy when searching for a match in the 10-print IAFIS database (containing more than 40 million subjects). Another study conducted by the Criminal Justice Information Services Division of the Federal Bureau of Investigation (FBI) [64] demonstrated a significant drop in performance when comparing 10-print flats against rolled prints in IAFIS. This was attributed to the system's inability to accurately process flat prints since the system was tuned to process rolled prints. The aforementioned studies highlight the importance of determining the cause for inferior performance when existing fingerprint matching systems are used to match rolled prints against plain/flat prints. It is interesting to note that a majority of the errors (misses) reported in [64] were due to the incorrect classification (caused by the RRI filtering process) of the query prints. Thus, the filtering constraints imposed by the matching infrastructure can significantly impact the identification accuracy.

In this report we describe a simple experiment that establishes the following: in the absence of filtering/indexing schemes, the matching performance of rolled prints against plain prints is as competitive as matching plain-against-plain or rolled-against-rolled. For this analysis, the publicly available NIST SD 29 database [3] was used ${ }^{3}$. This NIST fingerprint database offers complete paired fingerprint cards (of 216 different users) that include all ten rolled fingerprints as well as the corresponding plain/flat impressions (Fig. A.2). These are inked cards which have been converted into a digital format using a FBI complaint scanner. The paired fingerprint cards represent two sets of fingerprints of an individual captured at different time instances. The fingerprint data from the card-pairs facilitates any combination of "plain" and "rolled" images for comparison.

The rolled prints and the plain/flat impressions are segmented (i.e., separated) into individual fingerprints using the NIST segmentation algorithm. Fig. A.3 shows the rolled images of the left and right thumbs as well as the four plain impressions of the left hand after segmenting them from the fingerprint card depicted in Fig A.2. The four plain impressions are then segmented into individual plain prints (Fig. A.4).

\footnotetext{
${ }^{3}$ An earlier test conducted by NIST evaluated the matching performance of COTS matchers corresponding to twelve different vendors (including the vendor currently used by the US-VISIT system) on the NIST SD 29 dataset 65.
} 


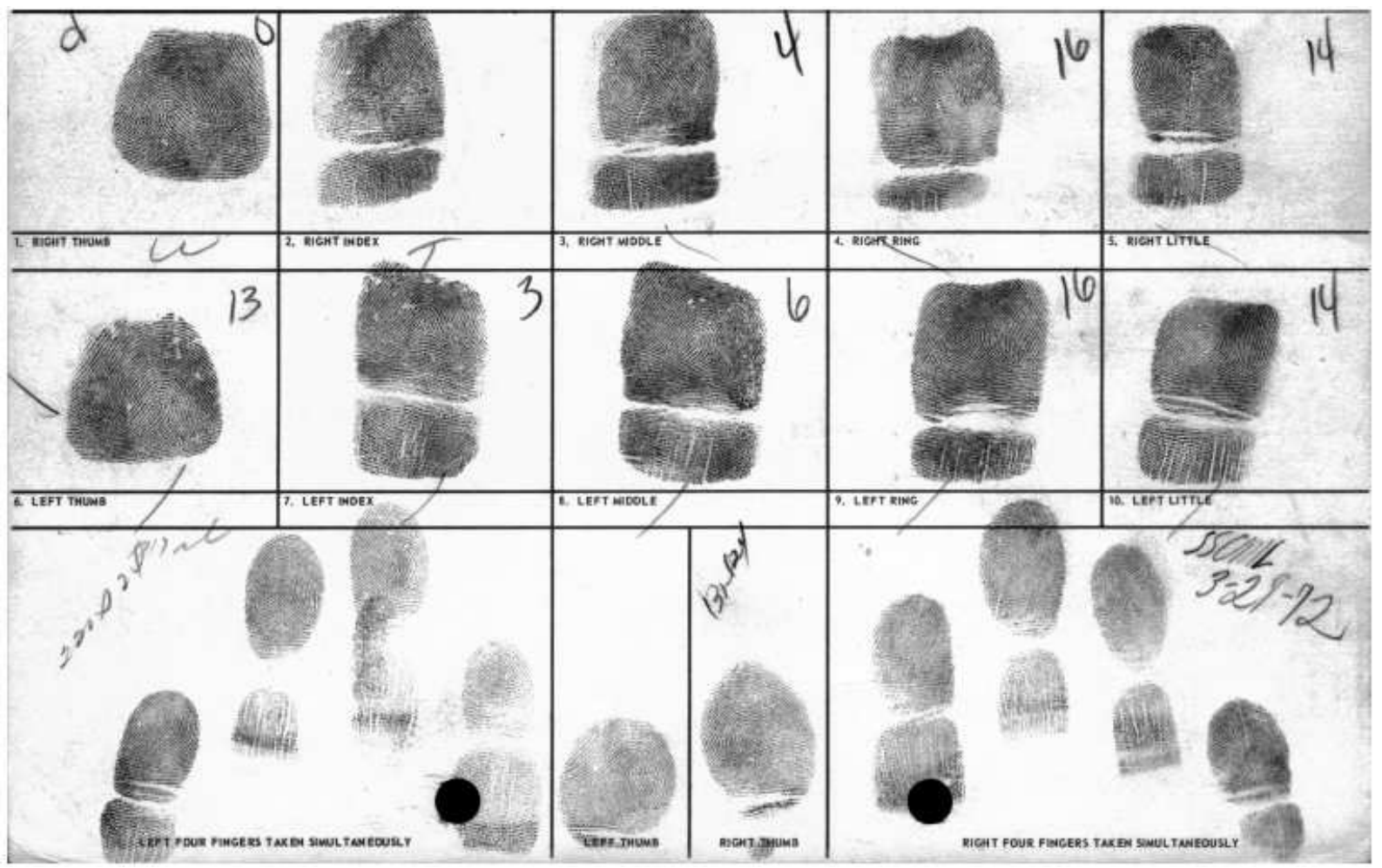

Figure A.2: Illustration of a fingerprint card available in the NIST Special Database 29 [3].
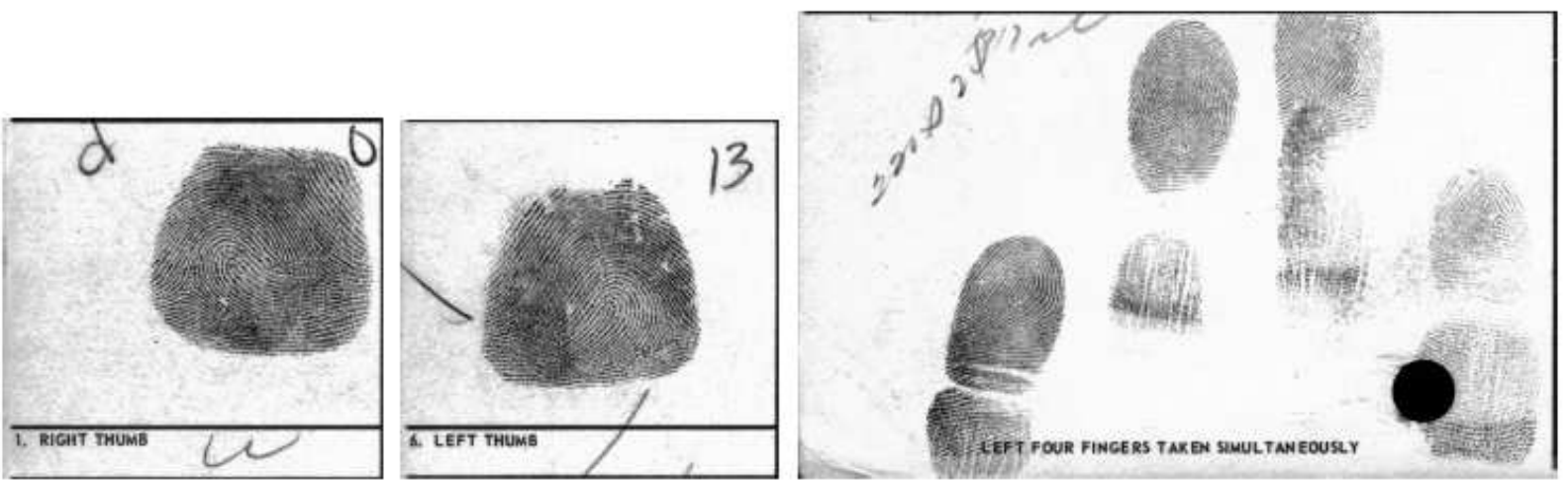

Figure A.3: Segmented images for right thumb, left thumb, and the four left plain impressions [3]. 

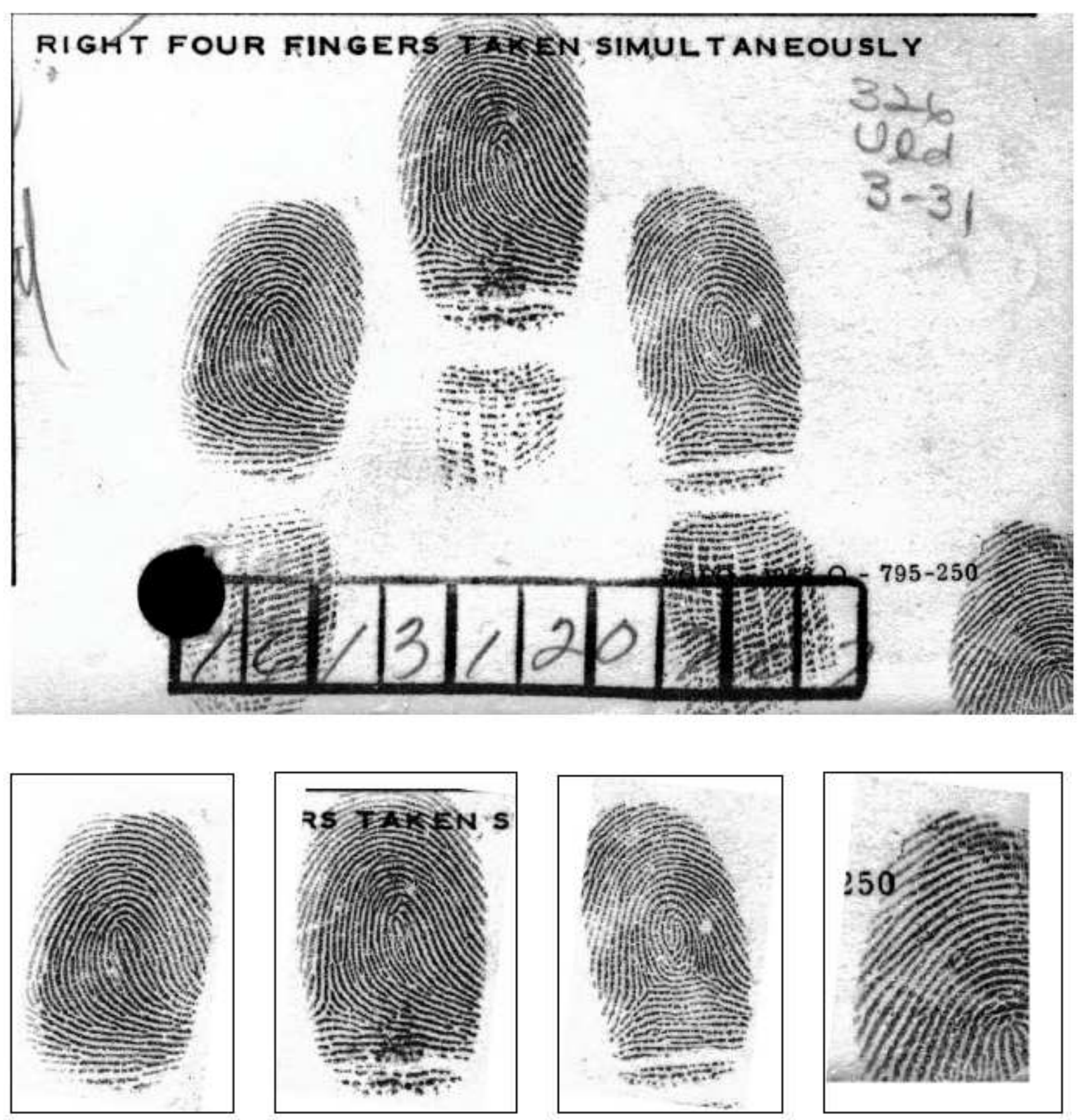

Figure A.4: Plain impressions are segmented into individual fingers [4]. 


\section{A.3 Experimental Results}

The 216 paired fingerprint cards are segmented to obtain 4320 (216 users *10 fingers/user *2 instances of each finger) rolled prints and 4320 segmented plain fingerprints 4 . The histogram of the number of minutiae points for each of the ten rolled and corresponding segmented plain fingerprints are shown in Fig. A.5. The histograms indicate the differences in minutiae count between the rolled and the plain prints of all ten fingers. This is directly related to the amount of fingerprint area exposed in the rolled and the plain prints. Due to the elaborate acquisition mechanism, the rolled prints contain more number of minutiae including points on the sides of the finger.

Three different experiments were conducted:

(a) Roll vs Roll (R2R);

(b) Plain vs Plain (P2P);

(c) Plain vs Roll (P2R).

In all three experiments, only fingerprints corresponding to the same finger digit were compared. Thus, for example, the fingerprint impression of the right thumb of a subject was compared only against other impressions of the right thumb.

1. Verification: In this experiment, the genuine and impostor match scores were generated for all three matching scenarios. The ROC curves presented in Fig. A.6 indicate that the rollversus-plain performance is comparable to the plain-versus-plain performance. Notwithstanding the fewer number of minutiae points detected by the NIST matcher on plain prints, the roll-versus-plain performance is still very competitive. The difference in performance between the roll-versus-roll scenario and the plain-versus-plain scenario may be attributed to the difference in minutiae points between the two sets of prints.

2. Identification: The data from 152 users was also used to analyze the identification performance. The CMC (Cumulative Match Characteristic) curves for all three matching scenarios are shown in Fig. A.7. The CMC graph plots the identification rate as a function of the number of top matches (ranks). The rank $\mathrm{K}$ identification rate denotes the probability that the correct identity occurs in the top K matches. Once again, these curves suggest

\footnotetext{
${ }^{4}$ Due to segmentation related issues, fingerprints of only 152 users were eventually used in the analysis
} 


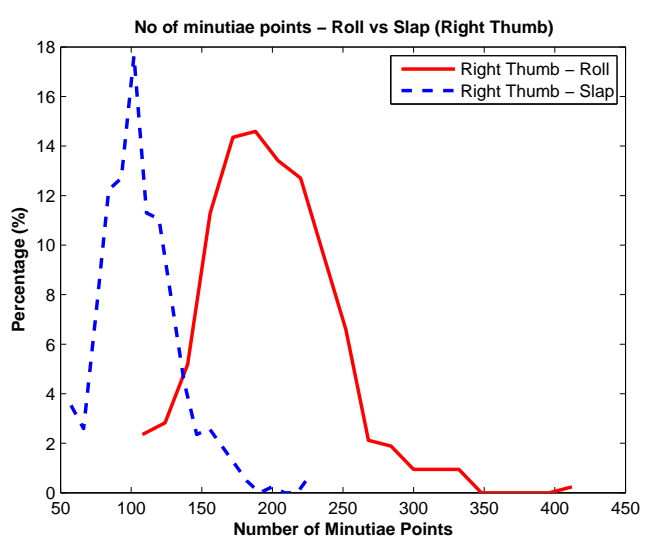

(a)

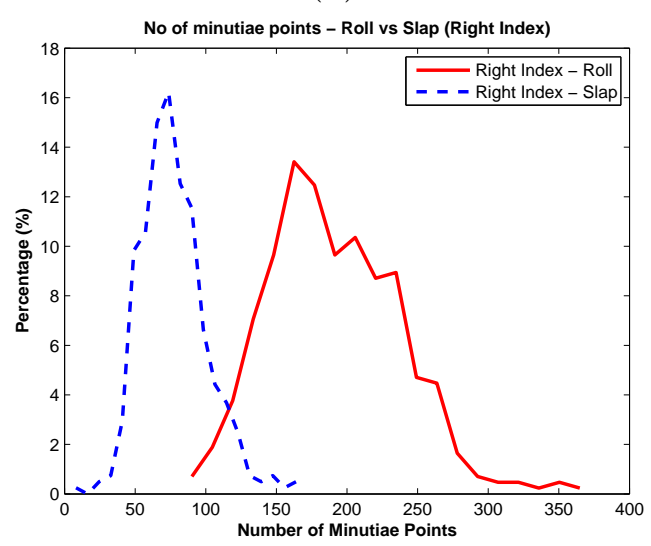

(c)

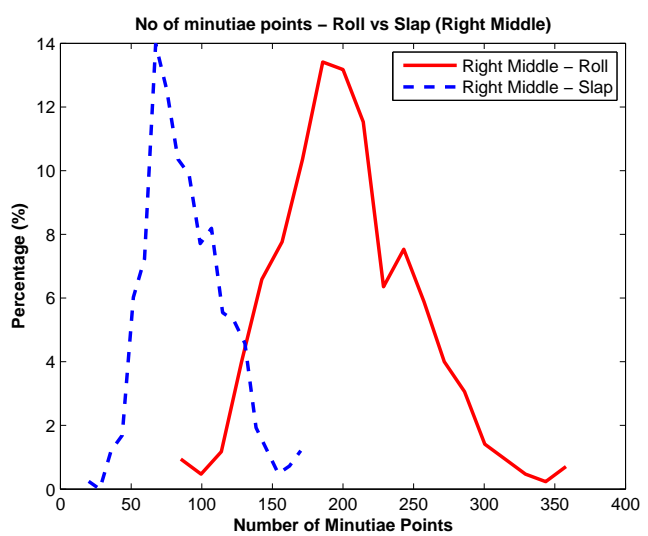

(e)

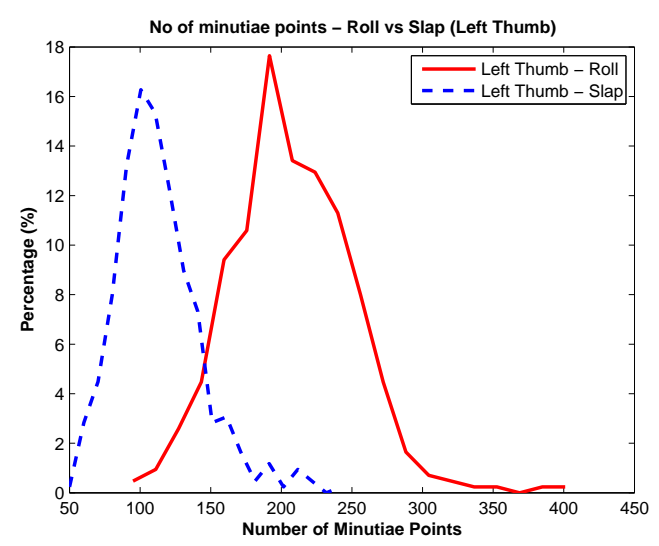

(b)

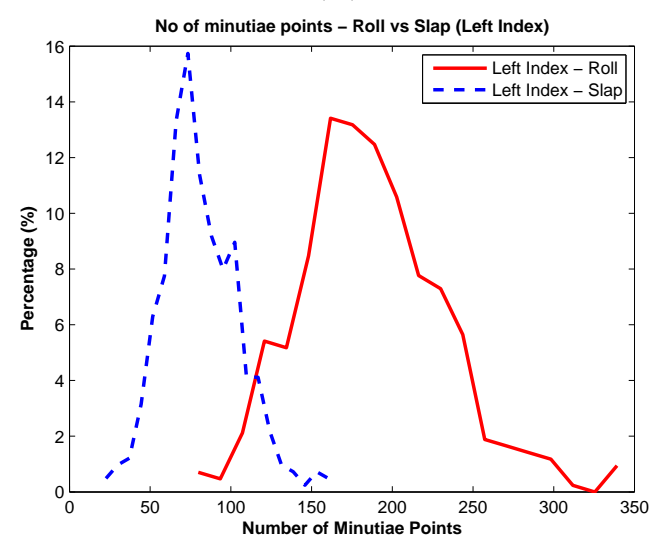

(d)

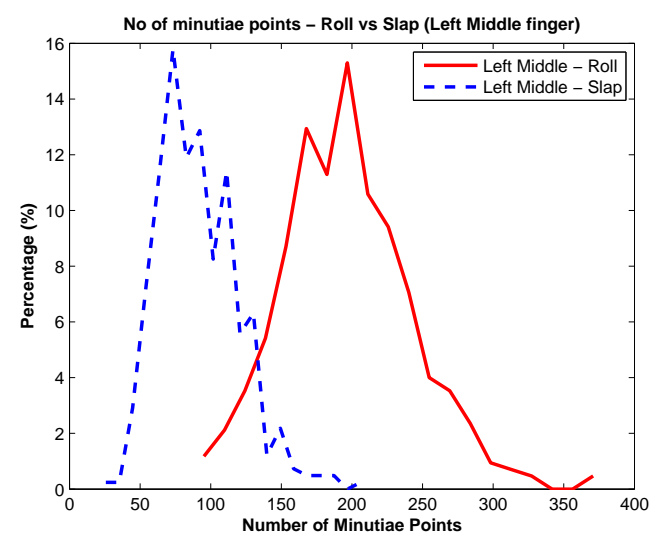

(f) 


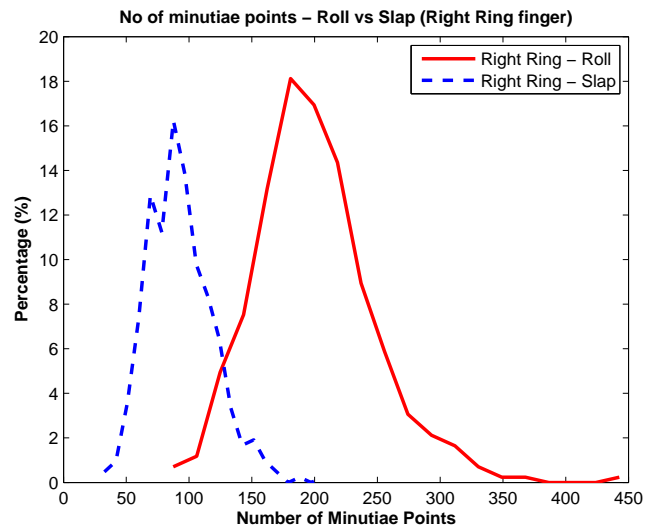

$(\mathrm{g})$

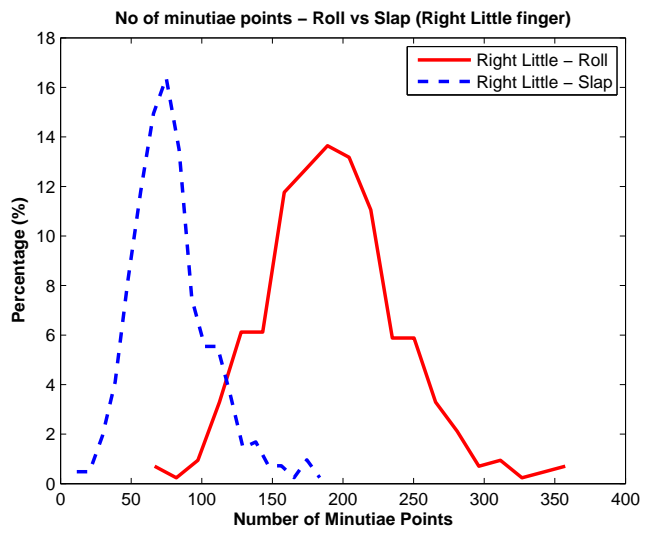

(i)

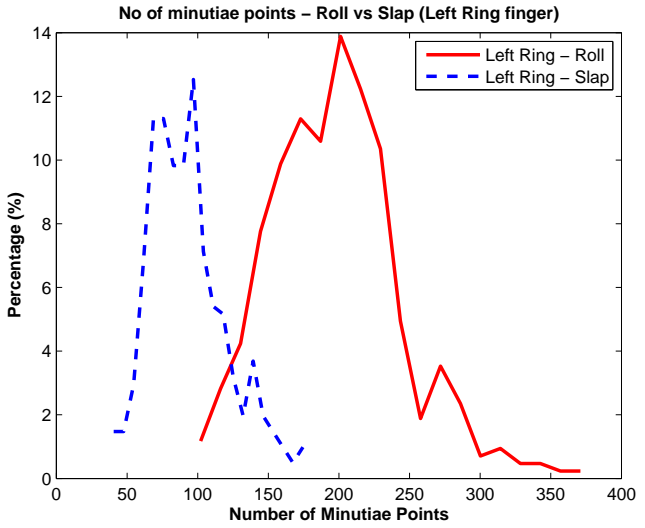

(h)

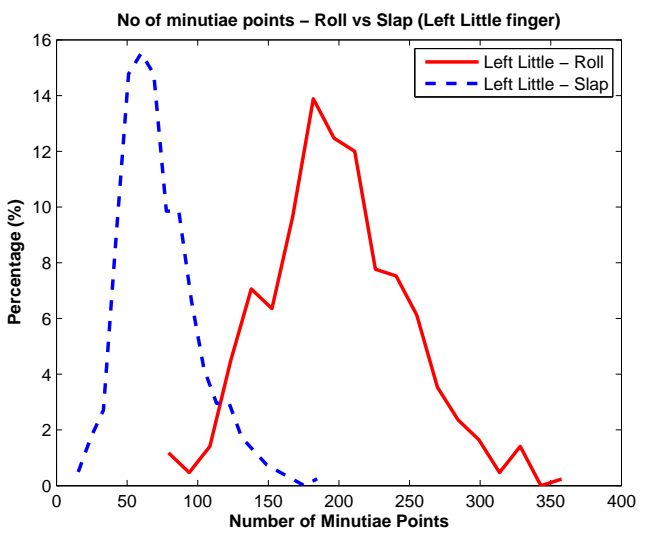

$(\mathrm{j})$

Figure A.5: Histograms illustrating the number of minutiae points for the roll and plain (slap) prints corresponding to the ten fingers. (a) Right Thumb, (b) Left Thumb, (c) Right Index finger, (d) Left Index finger, (e) Right Middle finger, (f) Left Middle finger, (g) Right Ring finger, (h) Left Ring finger, (i) Right Little finger, (j) Left Little finger. 


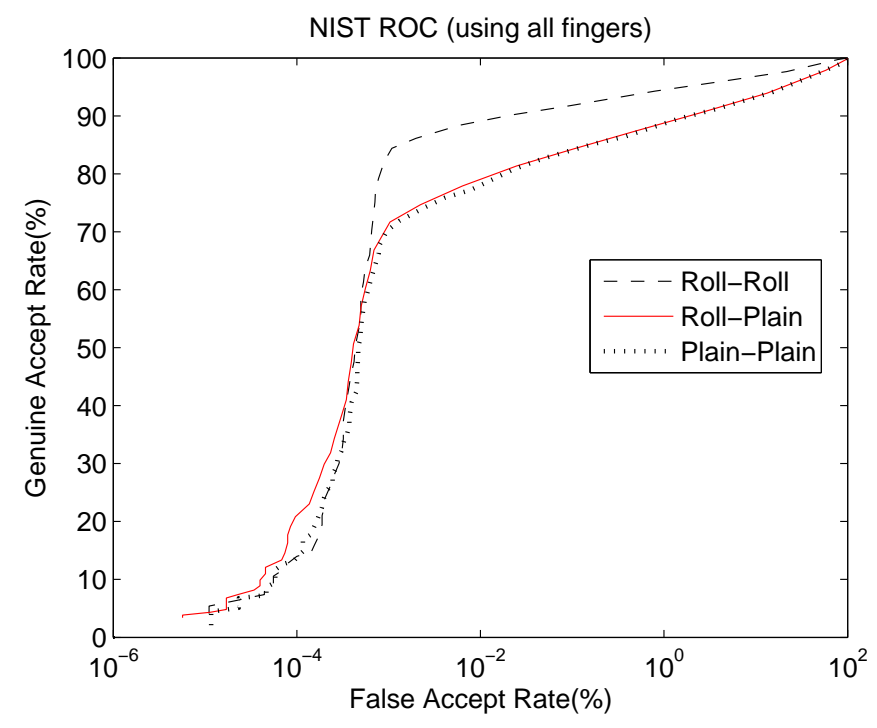

Figure A.6: The verification performance summarized using ROC curves for the SD29 database. The NIST matcher was used to generate the genuine and impostor scores. Note that the rollversus-roll matching performance is significantly superior possibly due to the large number of minutiae points available for matching.

the competitive performance of the roll-versus-plain scenario.

\section{A.4 Summary}

The ROC and CMC curves obtained using the NIST matcher suggest that matching plain prints against rolled prints (without any filtering) does not seem to drastically impact the matching accuracy. This appears to be the case with the images in the NIST SD 29 database. Thus, it is our contention that the recognition accuracy of the system is impacted more by the filtering scheme used to reduce the number of target prints. It may be the case that flat prints do not reveal sufficient pattern characteristics essential for accurate indexing. Hence, we believe that interoperability between prints acquired across different acquisition methodologies entail the adoption of new indexing schemes and not necessarily new matching algorithms. Furthermore, some of the geometric differences between such images can be accounted for by adopting simple non-linear calibration schemes 63$]$. 


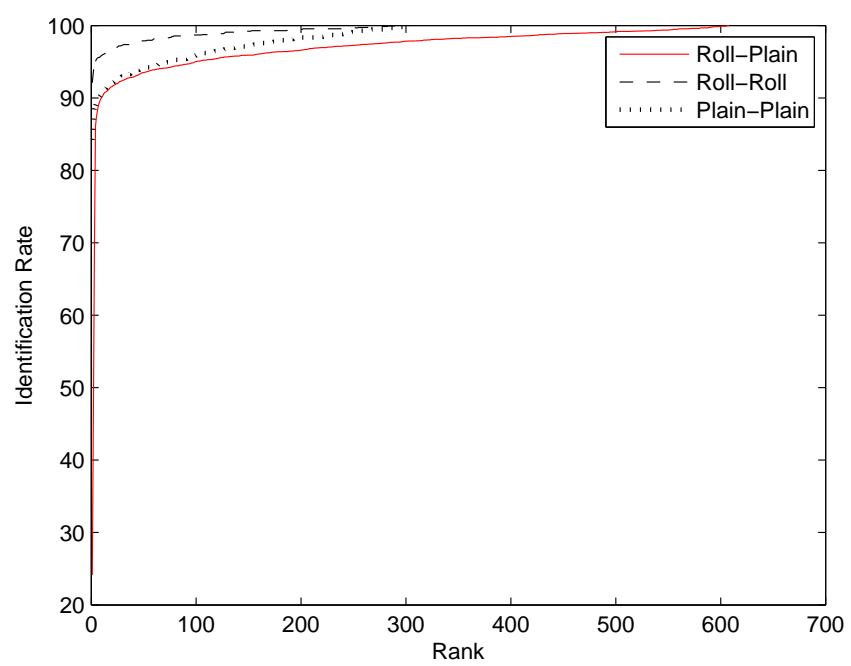

Figure A.7: CMC curves on the SD29 database as assessed using the NIST matcher. In this experiment, the roll-versus-plain identification accuracy is comparable to the other two scenarios, viz., roll-versus-roll and plain-versus-plain. 


\section{Appendix B}

\section{Fingerprint Matchers - NIST and}

\section{VeriFinger}

Two different minutiae-based matchers, Bozorth3 matcher developed by NIST and the VeriFinger (Neurotechnologija) matcher are used in our experiments.

Bozorth3 matcher is based on the location $(x, y)$ and orientation $(\theta)$ values of the minutiae. Tables are created for each of the to be matched fingerprints, based on the orientation and distances of minutia within the fingerprint. A inter-compatibility table is constructed from the tables generated for different fingerprints. Match score is calculated from the compatible minutia clusters of the inter-compatibility table [66]. Bozorth3 also utilizes the quality associated with each minutiae during matching. Only the top 150 best-quality minutiae are used for matching.

VeriFinger $4.1^{\circledR}$ is a minutiae based matcher which uses a proprietary fingerprint matching algorithm 1. VeriFinger provides matching details such as translation and rotation parameters along with a record of the matching minutiae pairs from the corresponding minutiae templates. VeriFinger utilizes a vast amount of information for matching which comprises of location, direction and curvature (type) of minutiae points (singular points), global ridge density and blocked orientations for the whole image. In our formulation, we have excluded both the blocked orientations and curvatures by using the VF_OMIT_BLOCKED_ORIENTATIONS_AND_CURVATURES option as we were not able to modify these parameters.

\footnotetext{
${ }^{1}$ http://www.neurotechnologija.com/
} 


\section{Appendix C}

\section{Ridge Feature-based matcher:}

\section{Fingercode}

In this matching scheme, both the global flow of ridge and valley structures and the local ridge characteristics are utilized to generate a short fixed length code for the fingerprints. Detailed description of this fingerprint matching algorithm is provided in [5].

The region of interest in a given fingerprint image is initially detected around a reference point (Fig. C.1). The core of the fingerprint represents the reference point. The region of interest is defined by the width and the number of bands around the reference point. Also, each band is divided by a certain number of orientations. Defining bands and orientations in the region of interest, leads to formation of sectors. Thus, the proposed scheme of feature extraction tessellates the region of interest of the given fingerprint image with respect to a reference point. A feature vector is generated by considering an ordered enumeration of the features extracted from the (local) information contained in each sector specified by the tessellation. The feature elements capture the local information while the ordered enumeration of the tessellation captures the invariant global relationships among the local patterns. The local discriminatory information in each sector is decomposed into separate components by gabor filterbanks. The average absolute deviation (AAD) from the mean of gray values in individual sectors of the filtered images is used to define the feature vector. The feature vector, called the FingerCode, is the collection of all the features (for every sector) in each filtered image. Matching is based on the Euclidean distance between the FingerCodes. 


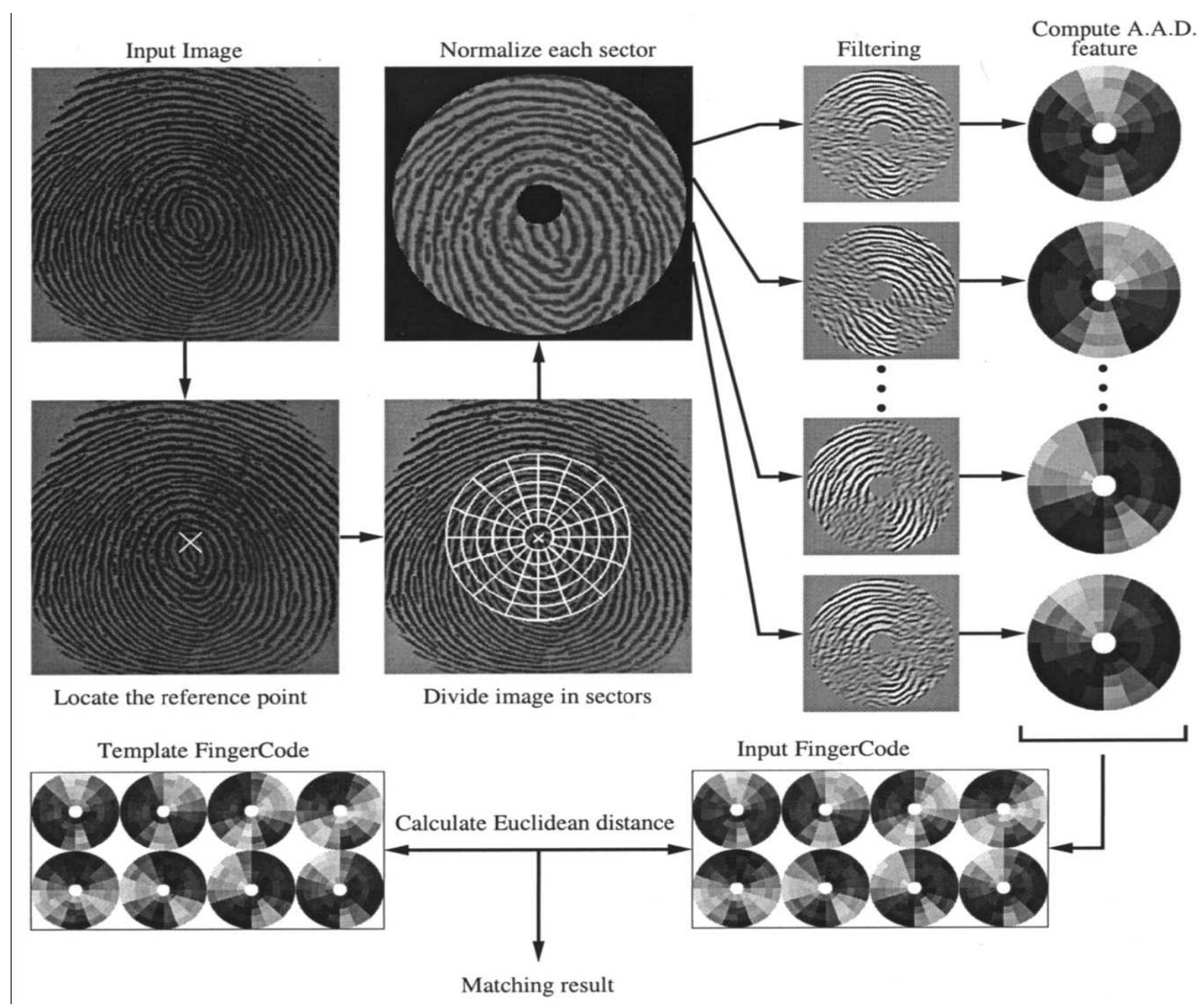

Figure C.1: Ridge feature-based matcher: Flowchart of fingerprint matching system (taken from [5]) 


\section{References}

[1] A. Hicklin and C. Reedy, "Implications of IDENT/IAFIS image quality study for visa fingerprint processing," Mitretek Systems, Tech. Rep., October 2002.

[2] B. Ulery, A. Hicklin, C. Watson, M. Indovina, and K. Kwong, "Slap fingerprint segmentation evaluation 2004," NIST and Mitretek Systems, NIST Interagency Report 7209, March 2005.

[3] C. Watson, "NIST Special Database 29 - plain and rolled images from paired fingerprint cards."

[4] E. Tabassi, C. L. Wilson, and C. I. Watson, "Fingerprint Image Quality," National Institute of Standards and Technology (NIST), Tech. Rep. 7151, August 2004.

[5] A. K. Jain, S. Prabhakar, L. Hong, and S. Pankanti, "Filterbank-based fingerprint matching," IEEE Transactions on Image Processing, vol. 9, no. 5, pp. 846-859, May 2000.

[6] D. Maltoni, D. Maio, A. K. Jain, and S. Prabhakar, Handbook of Fingerprint Recognition. Springer, July 2003.

[7] S. Pankanti, S. Prabhakar, and A. K. Jain, "On the individuality of fingerprints," IEEE Transactions on Pattern Analysis and Machine Intelligence, vol. 24, no. 8, pp. 1010-1025, 2002.

[8] L. Hong, Y. Wan, and A. Jain, "Fingerprint image enhancement: Algorithm and performance evaluation," IEEE Transactions on Pattern Analysis and Machine Intelligence, vol. 20, no. 8, pp. 777-789, August 1998.

[9] A. M. Bazen and S. H. Gerez, "Fingerprint matching by thin-plate spline modelling of elastic deformations," Pattern Recognition, vol. 36, no. 8, pp. 1859-1867, August 2003.

[10] Y. Chen, S. Dass, and A. Jain, "Fingerprint quality indices for predicting authentication performance," in Audio - and Video-based Biometric Person Authentication (AVBPA), vol. 3546, 2005, pp. 160-170.

[11] A. Ross and A. Jain, "Biometric sensor interoperability: A case study in fingerprints," in Proc. of International ECCV Workshop on Biometric Authentication (BioAW), vol. LNCS, no. 3087, May 2004, pp. 134-145.

[12] A. Martin, M. Przybocki, G. Doddington, and D. Reynolds, "The NIST speaker recognition evaluation - overview, methodology, systems, results, perspectives (1998)," Speech Communications, vol. 31, pp. 225-254, 2000. 
[13] International Biometric Group, "Independent testing of iris recognition technology (ITIRT) - Final Report," May 2005.

[14] P. J. Phillips, A. Martin, C. 1. Wilson, and M. Przybocki, "An introduction to evaluating biometric systems," IEEE Computer Society, vol. 33, pp. 56-63, February 2000.

[15] T. Faltemier and K. Bowyer, "Cross sensor 3D face recognition performance," in Proc. of SPIE Conference on Biometric Technology for Human Identification III, April 2006, pp. 620 201-1-8.

[16] F. Alonso-Fernandez, J. Fierrez-Aguilar, and J. Ortega-Garcia, "A review of schemes for fingerprint image quality computation," in 3rd COST-275 Workshop on Biometrics on the Internet, COST275, October 2005, pp. 3-6.

[17] J. Fierrez-Aguilar, Y. Chen, J. Ortega-Garcia, and A. K. Jain, "Incorporating image quality in multi-algorithm fingerprint verification," in International Conference on Biometrics (ICB06), 2006, pp. 213-220.

[18] F. Podio, J. Dunn, L. Reinert, C. Tilton, L. OGorman, P. Collier, M. Jerde, and B. Wirtz, "Common biometric exchange file format (CBEFF)," NIST, NIST Interagency Report 6529, 1999.

[19] R. M. Bolle, N. K. Ratha, A. Senior, and S. Pankanti, "Minutia template exchange format," in Proc. of IEEE Workshop on Automatic Identification Advanced Technologies, Summit, NJ, 1999, pp. 74-77.

[20] CJIS, "Electronic fingerprint transmission specification - Appendix F," Criminal Justice Information Services Division, Department of Justice - Federal Bureau of Investigation, Technical Report CJIS-RS-0010 (V7), January 1999.

[21] Secugen Biometric Solutions, "SEIR optic technology," White Paper, Secugen, 2004, (Available at http://www.secugen.com/company/ip.htm).

[22] M. S. Ennis, R. K. Rowe, S. P. Corcoran, and K. A. Nixon, "Multispectral sensing for highperformance fingerprint biometric imaging," White Paper, Lumidigm, Inc., (Available at http://www.lumidigm.com/lightPrint.html).

[23] X. Xia and L. O'Gorman, "Innovations in fingerprint capture devices," Pattern Recognition, vol. 36, no. 2, pp. 361-369, February 2003.

[24] H. H. Teng and S. C. Jo, "Method and apparatus for reduction of trapezoidal distortion and improvement of image sharpness in an optical image capturing system," US Patent 6,324,020 B1, November 272001.

[25] — - "High contrast low distortion optical acquisition system for image capturing," US Patent 6,381,347 B1, April 302002.

[26] A. Senior and R. Bolle, "Improved fingerprint matching by distortion removal," IEICE Transactions on Information and Systems, vol. E84-D, pp. 825-831, 2001.

[27] Z. Zhang, "Flexible camera calibration by viewing a plane from unknown orientations," in ICCV, 1999, pp. 666-673. 
[28] J.-M. Lavest, M. Viala, and M. Dhome, "Do we really need an accurate calibration pattern to achieve a reliable camera calibration?" in ECCV '98: Proceedings of the 5th European Conference on Computer Vision-Volume I, 1998, pp. 158-174.

[29] T. Clarke and J. Fryer, "The development of camera calibration methods and models," Photogrammetric Record, vol. 16, no. 91, pp. 51-66, April 1998.

[30] J. Heikkila and O. Silven, "A four-step camera calibration procedure with implicit image correction," in CVPR '97: Proceedings of the 1997 Conference on Computer Vision and Pattern Recognition (CVPR '97), 1997, pp. 1106-1112.

[31] A. Ross, S. Dass, and A. Jain, "A deformable model for fingerprint matching," Pattern Recognition, vol. 38, no. 1, pp. 95-103, January 2005.

[32] F. L. Bookstein, "Principal warps: Thin-plate splines and the decomposition of deformations," IEEE Transactions on Pattern Analysis and Machine Intelligence, vol. 11, no. 6, pp. 567-585, June 1989.

[33] K. Rohr, M. Fornefett, and H. S. Stiehl, "Approximating thin plate splines for elastic registration: Integration of landmark errors and orientation attributes," in 16th International Conference on Information Processing in Medical Imaging (IPMI), vol. 1613, June/July 1999, pp. 252-265.

[34] K. Rohr, H. Stiehl, R. Sprengel, W. Beil, T. Buzug, J. Weese, and M. Kuhn, "Point-based elastic registration of medical image data using approximating thin-plate splines," Lecture Notes in Computer Science, no. 1131, pp. 297-306, 1996.

[35] K. Rohr, R. Sprengel, and H. Stiehl, "Incorporation of landmark error ellipsoids for image registration based on approximating thin-plate splines," in Proc. Computer Assisted Radiology and Surgery (CAR'97), June 1997, pp. 234-239.

[36] K. Rohr, "Image registration based on thin-plate splines and local estimates of anisotropic landmark localization uncertainties." in Proceedings of the First International Conference on Medical Image Computing and Computer-Assisted Intervention (MICCAI), 1998, pp. $1174-1183$.

[37] A. C. Evans, W. Dai, D. L. Collins, P. Neelin, and S. Marrett, "Warping of a computerized 3-D atlas to match brain image volumes for quantitative neuroanatomical and functional analysis," in Proc. SPIE Medical Imaging V: Image Processing, Murray H. Loew; Ed., vol. 1445, June 1991, pp. 236-246.

[38] A. Almansa and L. Cohen, "Fingerprint image matching by minimization of a thin-plate energy using a two-step algorithm with auxiliary variables," in Fifth IEEE Workshop on Applications of Computer Vision, 2000, pp. 35-40.

[39] A. M. Bazen and S. H. Gerez, "Elastic minutiae matching by means of thin-plate spline models," in 16th International Conference on Pattern Recognition (ICPR'02), vol. 2, 2002, pp. 985-988.

[40] Y. Chen, S. Dass, A. Ross, and A. Jain, "Fingerprint deformation models using minutiae locations and orientations," in Seventh IEEE Workshops on Application of Computer Vision (WACV/MOTION'05), vol. 1, 2005, pp. 150-155. 
[41] A. Ross, S. Shah, and J. Shah, "Image versus feature mosaicing: A case study in fingerprints," in Proc. of SPIE Conference on Biometric Technology for Human Identification III, Orlando, FL, April 2006, pp. 620 208-1-12.

[42] A. Jain, S. Prabhakar, and A. Ross, "Fingerprint matching: Data acquisition and performance evaluation," Michigan State University, Technical Report MSU-CPS-99-14, March 1999.

[43] M. Yi-Sheng Yao, S. Pankanti, N. Hass, N. Ratha, and R. M. Bolle, "Quantifying quality: A case study in fingerprints," in Proc. of IEEE Conference on AutoID 2002, March 2002.

[44] R. Bolle, S. Pankanti, and Y. Yao, "System and method for determining the quality of fingerprint images," US Patent 5963656, 1999.

[45] J. Park and Y.-B. Kwon, "Image quality measure using sliced block distance as a graphical element," 5th International Workshop of Graphics Recognition: Recent Advances and Perspectives GREC, vol. 3088, pp. 211-222, 2003.

[46] N. K. Ratha, S. Chen, and A. K. Jain, "Adaptive flow orientation-based feature extraction in fingerprint images," Pattern Recognition, vol. 28, no. 11, pp. 1657-1672, 1995.

[47] L. Shen, A. Kot, and W. Koo, "Quality measure of fingerprint images," in Audio - and Video-based Biometric Person Authentication (AVBPA), 2001, pp. 266-271.

[48] G. Vallarino, G. Gianarelli, J. Baratttini, A. Gomez, A. Fernandez, and A. Pardo, "Performance improvement in a fingerprint classification system using anisotropic diffusion," CIARP - Iberoamerican Congress of Pattern Recognition, vol. LNCS 3287, pp. 582-588, 2004.

[49] C. Watson, C. L. Wilson, M. D. Garris, and R. M. McCabe, "User's guide to NIST image software," NIST, NIST Interagency Report 6813.

[50] N. K. Ratha and R. Bolle, "Research report - fingerprint image quality estimation," IBM Research Centre, Tech. Rep., 1999.

[51] E. Lim, X. Jiang, and W. Yau, "Fingerprint quality and validity analysis," in IEEE International Conference of Image Processing, vol. 1, 2002, pp. I-469 - I-472.

[52] S. Joun, H. Kim, Y. Chung, and D. Ahn, "An experimental study on measuring image quality of infant fingerprints," in Lecture Notes in Computer Science, vol. 2774, 2003, pp. 1261-1269.

[53] J. Qi, D. Abdurrachim, D. Li, and H. Kunieda, "A hybrid method for fingerprint image quality calculation," in Proceedings of the Fourth IEEE Workshop on Automatic Identification Advanced Technologies (AutoID 2005), October 2005, pp. 124-129.

[54] J. G. Daugman, "Uncertainty relation for resolution in space, spatial frequency, and orientation optimized by two-dimensional visual cortical filters," Journal of the Optical Society of America A, vol. 2, no. 7, pp. 1160-9, July 1985. 
[55] — - "High confidence visual recognition of persons by a test of statistical independence," IEEE Transactions on Pattern Analysis and Machine Intelligence, vol. 15, no. 11, pp. 11481161, November 1993.

[56] A. K. Jain, S. Prabhakar, L. Hong, and S. Pankanti, "FingerCode: A filterbank for fingerprint representation and matching," in Proceedings IEEE Conference on Computer Vision and Pattern Recognition, vol. 2, 1999, pp. 187-193.

[57] A. K. Jain, S. Prabhakar, and L. Hong, "A multichannel approach to fingerprint classification," IEEE Transactions on Pattern Analysis and Machine Intelligence, vol. 21, no. 4, pp. 348-359, 1999.

[58] J. Yang, L. Liu, T. Jiang, and Y. Fan, "A modified Gabor filter design method for fingerprint image enhancement," Pattern Recognition Letters, vol. 24, no. 12, pp. 1805-1817, 2003.

[59] D. Simon-Zorita, J. Ortega-Garcia, J. Fierrez-Aguilar, and J. Gonzalez-Rodriguez, "Image quality and position variability assessment in minutiae-based fingerprint verification," IEE Proc. Vision, Image and Signal Processing (VISP), Special Issue on Biometrics on the Internet, vol. 150, no. 6, pp. 402-408, December 2003.

[60] D. Simon-Zorita, J. Ortega-Garcia, M. Sanchez-Asenjo, and J. Gonzalez-Rodriguez, "Minutiae-based enhanced fingerprint verification assessment relaying on image quality factors," in International Conference of Image Processing ICIP, 2003, pp. II: 891-894.

[61] P. Grother and E. Tabassi, "Performance of biometric quality measures." National Institute of Standards and Technology, April 2006.

[62] J. Fierrez-Aguilar, L. Muoz-Serrano, F. Alonso-Fernandez, and J. Ortega-Garcia, "On the effects of image quality degradation on minutiae and ridge-based automatic fingerprint recognition," in IEEE International Carnahan Conference on Security Technology, October 2005, pp. $79-82$.

[63] A. Ross and R. Nadgir, "A calibration model for fingerprint sensor interoperability," in Proc. of SPIE Conference on Biometric Technology for Human Identification III, Orlando, FL, April 2006, pp. 620 201-1-8.

[64] U.S. Department of Justice, "National fingerprint-based applicant check study (N-FACS)," Criminal Justice Information Services Division - Federal Bureau of Investigation, Technical Report IAFIS-DOC-07054-1.0, April 2004.

[65] C. Watson, C. Wilson, K. Marshall, M. Indovina, and R. Snelick, "Studies of one-to-one fingerprint matching with vendor SDK matchers," NIST Interagency Report 7221, April 2005.

[66] C. I. Watson, M. D. Garris, E. Tabassi, C. L. Wilson, R. M. McCabe, and S. Janet, "User's guide to NIST fingerprint image software 2 (NFIS2)," NIST, Tech. Rep., (Available at http://fingerprint.nist.gov/NFIS/). 